\title{
ON AN OPERATOR APPROACH TO INTERPOLATION PROBLEMS FOR STIELTJES FUNCTIONS
}

\author{
V. BOLOTNIKOV AND L. SAKHNOVICH
}

A general interpolation problem for operator-valued Stieltjes functions is studied using V. P. Potapov's method of fundamental matrix inequalities and the method of operator identities. The solvability criterion is established and under certain restrictions the set of all solutions is parametrized in terms of a linear fractional transformation. As applications of a general theory, a number of classical and new interpolation problems are considered.

\section{Introduction}

Classical interpolation problems (Schur, Nevanlinna-Pick, Carathéodory-Féjer problems, the moment problem et.c.) and their various generalizations were studied using several different approaches and methods (see e.g., [8] for short historical survey). It turns out that the interpolation data of each such problem satisfies a certain operator identity. The structure of this identity turns to be similar (and often, the same) for quite different problems. This allows one to consider a whole circle of problems in a unified way [19], [23]. On the other hand, the operator identities themselves are useful for applications [23], [24].

In [13] a general interpolation problem generated by an operator identity has been considered in the Nevanlinna class of matrix valued functions analytic and with the nonnegative imaginary part in the upper half-plane $\mathbb{C}_{+}$. It was shown that the classical NevanlinnaPick and Carathéodory-Féjer interpolation problems are particular cases of this problem as well as the Hamburger moment problem, the Krein extension problem for positive functions on the interval and so on. In this paper we consider a similar problem for the Stieltjes functions (see Definition 2.1) which form an important subclass of the Nevanlinna class. The Stieltjes functions and their role in applications have been studied in [14]. The interpolation problems for Stieltjes functions has been considered in [21] (the scalar case), [11], [2], [5] (the matrix-valued case). We refer also to [5], [10] where interpolation problems for Stieltjes functions are interpreted in terms of integral representations of a pair of nonnegative operators.

Throughout the paper $\mathcal{H}$ and $\widetilde{\mathcal{H}}$ are the separable Hilbert spaces with the inner 
products $\langle,\rangle_{\mathcal{H}}$ and $\langle,\rangle_{\widetilde{\mathcal{H}}}$ respectively and $\{\mathcal{H} ; \widetilde{\mathcal{H}}\}$ stands for the set of bounded linear operators acting from $\mathcal{H}$ to $\widetilde{\mathcal{H}}$.

Let $A \in\{\mathcal{H} ; \mathcal{H}\}, \quad B \in\{\mathcal{H} ; \widetilde{\mathcal{H}}\}$ and $C \in\{\widetilde{\mathcal{H}} ; \mathcal{H}\}$ be operators such that the spectrum of the pencil $A-z C B$, is an at most countable closed set,

$$
\mathcal{Z}=\operatorname{spec}(A-z C B):=\left\{z_{i} \in \mathbb{C}:\left(A-z_{i} C B\right)^{-1} \notin\{\mathcal{H} ; \mathcal{H}\}\right\}
$$

and has no accumulation points on the positive semi-axis $\mathbb{R}_{+}$. Then the function

$$
\Gamma(z)=(A-z C B)^{-1}
$$

is $\{\mathcal{H} ; \mathcal{H}\}$-valued and analytic for all $z \in \mathbb{C} \backslash \mathcal{Z}$ and in particular, it is analytic on $\mathbb{R}_{+}$except at an at most countable set $\Lambda$ of isolated points

$$
\Lambda=\mathcal{Z} \cap \mathbb{R}_{+}=\left\{\lambda_{i} \geq 0:\left(A-\lambda_{i} C B\right)^{-1} \notin\{\mathcal{H} ; \mathcal{H}\}\right\}
$$

Let $\Pi_{2}$ be arbitrary operator from $\{\mathcal{G} ; \mathcal{H}\}$ and let $\mathcal{E}$ denote the set of all nondecreasing $\{\mathcal{G} ; \mathcal{G}\}$-valued functions $\sigma(\lambda)$ on $\mathbb{R}_{+} \backslash \Lambda$ such that the integrals

$$
K_{\sigma}:=\int_{\mathbb{R}_{+} \backslash \Lambda} \Gamma(\lambda) \Pi_{2} d \sigma(\lambda) \Pi_{2}^{*} \Gamma(\lambda)^{*}, \quad \widetilde{K}_{\sigma}:=\int_{\mathbb{R}_{+} \backslash \Lambda} \lambda B \Gamma(\lambda) \Pi_{2} d \sigma(\lambda) \Pi_{2}^{*} \Gamma(\lambda)^{*} B^{*}
$$

and

$$
\mathbf{J}=\int_{\mathbb{R}_{+} \backslash \Lambda} \frac{d \sigma(\lambda)}{\lambda+1}
$$

converge in the weak sense. For $\sigma \in \mathcal{E}$, the integral

$$
\Pi_{1, \sigma}:=\int_{\mathbb{R}_{+} \backslash \Lambda} B \Gamma(\lambda) \Pi_{2} d \sigma(\lambda)
$$

converges in the weak sense for every $\sigma(\lambda) \in \mathcal{E}$. To show this, consider the space $\mathcal{L}$ consisting of $\mathcal{G}$-valued functions $g(\lambda)$ with the following inner product

$$
\langle g, g\rangle_{\mathcal{L}}:=\int_{\mathbb{R}_{+} \backslash \Lambda}(d \sigma(\lambda) g(\lambda), g(\lambda))_{\mathcal{G}}
$$

Since integrals (1.4), (1.5) converge, it follows that for every $f \in \widetilde{\mathcal{H}}$ and $h \in \mathcal{G}$,

$$
\sqrt{\lambda+1} \Pi_{2}^{*} \Gamma(\lambda)^{*} B^{*} f \in \mathcal{L} \quad \text { and } \quad \frac{h}{\sqrt{\lambda+1}} \in \mathcal{L}
$$

which in turn implies the convergence of

$$
\int_{\mathbb{R}_{+} \backslash \Lambda}\left\langle d \sigma(\lambda) \frac{h}{\sqrt{\lambda+1}}, \sqrt{\lambda+1} \Pi_{2}^{*} \Gamma(\lambda)^{*} B^{*} f\right\rangle_{\mathcal{G}}
$$

and therefore, the weak convergence of (1.6). The following identity

$$
A K_{\sigma} B^{*}-C \widetilde{K}_{\sigma}=\Pi_{2} \Pi_{1, \sigma}^{*}
$$


is an immediate consequence of (1.4) and (1.6):

$$
\begin{aligned}
A K_{\sigma} B^{*}-C \widetilde{K}_{\sigma} & =\int_{\mathbb{R}_{+} \backslash \Lambda}(A-\lambda C B) \Gamma(\lambda) \Pi_{2} d \sigma(\lambda) \Pi_{2}^{*} \Gamma(\lambda)^{*} B^{*} \\
& =\Pi_{2} \int_{\mathbb{R}_{+} \backslash \Lambda} d \sigma(\lambda) \Pi_{2}^{*} \Gamma(\lambda)^{*} B^{*}=\Pi_{2} \Pi_{1, \sigma}^{*} .
\end{aligned}
$$

Let us introduce the operators $K \in\{\mathcal{H} ; \mathcal{H}\}, \widetilde{\mathcal{K}} \in\{\widetilde{\mathcal{H}} ; \widetilde{\mathcal{H}}\}$ and $\Pi_{1} \in\{\widetilde{\mathcal{H}} ; \mathcal{G}\}$ by

$$
\begin{aligned}
K & =K_{\sigma}+P_{\infty}+\sum_{i}\left(P_{i}+F_{i} F_{i}^{*}\right) \\
\widetilde{K} & =\widetilde{K}_{\sigma}+\widetilde{F} \widetilde{F}^{*}+\widetilde{P}_{\infty}+\sum_{i} \lambda_{i} B\left(P_{i}+F_{i} F_{i}^{*}\right) B^{*} \\
\Pi_{1} & =\Pi_{1, \sigma}-\widetilde{F} \gamma^{\frac{1}{2}}+\sum_{i} B F_{i} x_{i}^{\frac{1}{2}}
\end{aligned}
$$

where $\lambda_{i}$ are points from $\Lambda$; where nonnegative operators $P_{\infty} \in\{\mathcal{H} ; \mathcal{H}\}, \widetilde{P}_{\infty} \in\{\widetilde{\mathcal{H}} ; \widetilde{\mathcal{H}}\}$, $\gamma \in\{\mathcal{G} ; \mathcal{G}\}$ and the operator $\widetilde{F} \in\{\mathcal{G} ; \widetilde{\mathcal{H}}\}$ are such that

$$
B P_{\infty}=0, \quad C \widetilde{P}_{\infty}=0, \quad C \widetilde{F}=\Pi_{2} \gamma^{\frac{1}{2}}
$$

and where nonnegative operators $P_{i} \in\{\mathcal{H} ; \mathcal{H}\}, x_{i} \in\{\mathcal{G} ; \mathcal{G}\}$ and operators $F_{i} \in\{\mathcal{G} ; \mathcal{H}\}$ are subject to

$$
\left(A-\lambda_{i} C B\right) P_{i}=0, \quad\left(A-\lambda_{i} C B\right) F_{i}=\Pi_{2} x_{i}^{\frac{1}{2}} .
$$

It turns out that every triple $\left\{K, \widetilde{K}, \Pi_{1}\right\}$ of the form (1.8)-(1.12) satisfies the identity

$$
A K B^{*}-C \widetilde{K}=\Pi_{2} \Pi_{1}^{*}
$$

Indeed, it follows from (1.7)-(1.12) that

$$
\begin{aligned}
A K B^{*}-C \widetilde{K} & =\Pi_{2} \Pi_{1, \sigma}^{*}-C \widetilde{F} \widetilde{F}^{*}+\sum_{i}\left(A-\lambda_{i} C B\right) F_{i} F_{i}^{*} B^{*} \\
& =\Pi_{2} \Pi_{1, \sigma}^{*}-\Pi_{2} \gamma^{\frac{1}{2}} \widetilde{F}^{*}+\Pi_{2} \sum_{i} x_{i}^{\frac{1}{2}} F_{i}^{*} B^{*}=\Pi_{2} \Pi_{1}^{*} .
\end{aligned}
$$

Moreover, the operator identity (1.13) suggests the following interpolation problem.

Problem 1.1 Given operators

$$
\begin{gathered}
A \in\{\mathcal{H} ; \mathcal{H}\}, \quad B \in\{\mathcal{H} ; \widetilde{\mathcal{H}}\}, \quad C \in\{\widetilde{\mathcal{H}}, \mathcal{H}\}, \quad \Pi_{2} \in\{\mathcal{G} ; \mathcal{H}\} \\
K \in\{\mathcal{H} ; \mathcal{H}\}, \quad \widetilde{K} \in\{\widetilde{\mathcal{H}} ; \widetilde{\mathcal{H}}\}, \quad \Pi_{1} \in\{\mathcal{G} ; \widetilde{\mathcal{H}}\}
\end{gathered}
$$

satisfying the operator identity (1.13), to describe all functions $\sigma \in \mathcal{E}$ which give the representations (1.8)-(1.12).

The presence of the operators $P_{i}$ and $F_{i}$ in (1.8)-(1.10) is conditioned by singular points of the function $\Gamma$ (given by (1.2)) on the positive half-axis. Note that for the case when $\Gamma$ is analytic on $\mathbb{R}_{+}$the set $\Lambda$ is empty and Problem 1.1 is simplified as follows. 
Problem 1.2 Given operators (1.14) satisfying the operator identity (1.13), to describe all functions $\sigma \in \mathcal{E}$ which give the representations

$$
\begin{aligned}
K & =\int_{0}^{\infty} \Gamma(\lambda) \Pi_{2} d \sigma(\lambda) \Pi_{2}^{*} \Gamma(\lambda)^{*}+P_{\infty} \\
\widetilde{K} & =\int_{0}^{\infty} \lambda B \Gamma(\lambda) \Pi_{2} d \sigma(\lambda) \Pi_{2}^{*} \Gamma(\lambda)^{*} B^{*}+\widetilde{F} \widetilde{F}^{*}+\widetilde{P}_{\infty} \\
\Pi_{1} & =\int_{0}^{\infty} B \Gamma(\lambda) \Pi_{2} d \sigma(\lambda)-\widetilde{F} \gamma^{\frac{1}{2}}
\end{aligned}
$$

where nonnegative operators $P_{\infty} \in\{\mathcal{H} ; \mathcal{H}\}, \widetilde{P}_{\infty} \in\{\widetilde{\mathcal{H}} ; \widetilde{\mathcal{H}}\}, \gamma \in\{\mathcal{G} ; \mathcal{G}\}$ and the operator $\widetilde{F} \in\{\mathcal{G} ; \widetilde{\mathcal{H}}\}$ are subject to (1.11).

For a number of interpolation problems (e.g., Stieltjes moment problem, Krein's extension problem on $\mathbb{R}_{+}$; see Sections 10 and 11) the operators $B$ and $C$ have zero kernels and $\gamma=0$. For such a case the additional terms $P_{\infty}, \widetilde{P}_{\infty}$ and $\widetilde{F}$ do not appear in (1.15)-(1.17), and Problem 1.2 deals in fact with integral representations of given operators $K, \widetilde{K}$ and $\Pi_{1}$ satisfying the operator identity (1.13).

The solvability criterion of Problem 1.1 is established by the following

Theorem 1.3 Let the operator $\Pi_{2}^{*}$ be surjective: $\operatorname{Ran} \Pi_{2}^{*}=\mathcal{G}$. Then Problem 1.1 has a solution if and only if $K \geq 0$ and $\bar{K} \geq 0$.

The necessary part of the theorem follows immediately from (1.8), (1.9); the sufficiency will be proved in Section 5.

The paper is organized as follows: in Section 2 we construct the abstract analogues of V. P. Potapov's fundamental matrix inequalities. The equivalence of Problem 1.1 to the system of the constructed inequalities is established in Sections 3 and 4. The parametrization of all solutions in terms of the linear fractional transformation is given in Section 7 under assumption that $K$ and $\widetilde{K}$ are boundedly invertible. The matrix of coefficients of this linear fractional transformation is presented in Section 6. Sections 8-10 are devoted to concrete interpolation problems illustrating the general theory: two-sided residue interpolation problem of Nudelman type [22] (which generalizes classic Nevanlinna-Pick and Carathéodory-Féjer problems), boundary interpolation problem of Loewner type, Stieltjes moment problem. In Section 11 we establish analogues of Krein's extension problem for a number of integro-differential operators which appear and play a central role in the spectral analysis of the matrix string [24].

\section{Fundamental Matrix Inequalities}

In this section we explain why Problem 1.1 can be considered as some general interpolation problem in the Stieltjes class of analytic operator-valued functions. Following Potapov's method we describe all solutions $d \sigma$ of Problem 1.1 in terms of the system of the fundamental matrix inequalities. We begin with the definition and some needed results concerning Stieltjes functions. 
Definition 2.1 $A\{\mathcal{G} ; \mathcal{G}\}$-valued function $s(z)$ is said to be in the Stieltjes class $\mathcal{S}(\mathcal{G})$ if it is analytic in the complex plane with a cut along the positive semi-axis $\mathbb{C} \backslash \mathbb{R}_{+}$and such that

$$
\frac{s(z)-s(z)^{*}}{z-\bar{z}} \geq 0 \quad(\Im z \neq 0) \quad \text { and } \quad s(x) \geq 0 \quad(x<0) .
$$

The class $\mathcal{S}(\mathcal{G})$ is a subclass of the Nevanlinna class $\mathcal{N}(\mathcal{G})$ which have been already mentioned in Section 1 and which consists of all $\{\mathcal{G} ; \mathcal{G}\}$-valued functions $s(z)$ analytic in $\mathbb{C} \backslash \mathbb{R}$ and satisfying the first inequality in (2.1) and the symmetry relation

$$
s(z)=s(\bar{z})^{*} \quad(z \notin \mathbb{R}) .
$$

Note that the latter symmetry relation holds for any Stieltjes function $s$ automatically, since $s$ takes selfadjoint values on $\mathbb{R}_{-}$. The following theorem exhibits an equivalent definition of the class $\mathcal{S}(\mathcal{G})$.

Theorem 2.2 The function $s(z)$ analytic in $\mathbb{C} \backslash \mathbb{R}_{+}$belongs to $\mathcal{S}(\mathcal{G})$ if and only if

$$
\frac{s(z)-s(z)^{*}}{z-\bar{z}} \geq 0 \quad \text { and } \quad \frac{z s(z)-\bar{z} s(z)^{*}}{z-\bar{z}} \geq 0 \quad \text { for } \Im z \neq 0 .
$$

In other words, $s(z) \in \mathcal{S}(\mathcal{G})$ if and only if $s(z) \in \mathcal{N}(\mathcal{G})$ and $z s(z) \in \mathcal{N}(\mathcal{G})$.

Theorem 2.3 The function $s(z)$ belongs to $\mathcal{S}(\mathcal{G})$ if and only if it admits the integral representation

$$
s(z)=\gamma+\int_{0}^{\infty} \frac{d \sigma(\lambda)}{\lambda-z}
$$

where $\gamma$ is a nonnegative operator from $\{\mathcal{G} ; \mathcal{G}\}$ and $\sigma$ is a nondecreasing $\{\mathcal{G} ; \mathcal{G}\}$-valued function such that

$$
\left\|\int_{0}^{\infty} \frac{d \sigma(\lambda)}{\lambda+1}\right\|<\infty
$$

and integrals in (2.2), (2.3) converge in the weak sense. The measure do is uniquely determined from the function $s$ by the Stieltjes inversion formula

$$
\sigma\left(\lambda_{2}\right)-\sigma\left(\lambda_{1}\right)=\frac{1}{\pi} \lim _{\varepsilon \rightarrow+0} \int_{\lambda_{1}}^{\lambda_{2}} \Im s(x+i \varepsilon) d x .
$$

which is also meant in the weak sense.

Two last theorems can be easily deduced from their scalar analogues [21, p.392]. Note that the uniform convergence of the integrals in (2.2), (2.3) can be provided by certain additional restrictions on $s$.

Theorem 2.4 Let $s \in \mathcal{S}(\mathcal{G})$ and let $\sup _{y \geq 1}\|\Im s(i y)\|<\infty$. Then the integrals in (2.2), (2.3) converge uniformly. If moreover, s satisfies

$$
\sup _{y \geq 1}\|y s(i y)\|<\infty
$$


then it admits a representation

$$
s(z)=\int_{0}^{\infty} \frac{d \sigma(\lambda)}{\lambda-z}
$$

where $\sigma$ is a nondecreasing $\{\mathcal{G} ; \mathcal{G}\}$-valued function such that

$$
\left\|\int_{0}^{\infty} d \sigma(\lambda)\right\|<\infty
$$

and the integrals in (2.6), (2.7) converge uniformly.

The latter theorem is an immediate consequence of Theorems 4.7 and 4.8 from [6, p.25] where the results claimed in Theorem 2.4 were obtained for Nevanlinna functions $s \in \mathcal{N}(\mathcal{G})$ and representing measures $d \sigma$ supported by the whole real axis. Since any Stieltjes function $s$ can be characterized as a Nevanlinna function taking nonnegative values on $\mathbb{R}_{-}$, the Stieltjes inversion formula (2.4) implies, that the representing measure $d \sigma$ increases only on $\mathbb{R}_{+}$, and assertions of Theorem 2.4 follow from the corresponding results in [6].

To every triple of representations $(1.8),(1.10)$ we associate the $\{\mathcal{G} ; \mathcal{G}\}$-valued function

$$
s(z)=\gamma+\int_{\mathbb{R}_{+} \backslash \Lambda} \frac{d \sigma(\lambda)}{\lambda-z}+\sum_{\lambda_{i} \in \Lambda} \frac{x_{i}}{\lambda_{i}-z} .
$$

Since

$$
\begin{aligned}
\frac{s(z)-s(z)^{*}}{z-\bar{z}} & =\int_{\mathbb{R}_{+} \backslash \Lambda} \frac{d \sigma(\lambda)}{|\lambda-z|^{2}}+\sum_{\lambda_{i} \in \Lambda} \frac{x_{i}}{\left|\lambda_{i}-z\right|^{2}} \geq 0 \\
\frac{z s(z)-\bar{z} s(z)^{*}}{z-\bar{z}} & =\gamma+\int_{\mathbb{R}_{+} \backslash \Lambda} \frac{\lambda d \sigma(\lambda)}{|\lambda-z|^{2}}+\sum_{\lambda_{i} \in \Lambda} \frac{\lambda_{i} x_{i}}{\left|\lambda_{i}-z\right|^{2}} \geq 0,
\end{aligned}
$$

the function $s$ given by $(2.8)$ belongs to the Stieltjes class $\mathcal{S}(\mathcal{G})$ by Theorem 2.2.

It turns out that Problem 1.1 is equivalent to a system of certain inequalities with respect to $s \in \mathcal{S}(\mathcal{G})$. The next theorem establishes only the necessary part of this equivalence.

Theorem 2.5 Let $d \sigma \geq 0$ be a measure from (1.8), (1.10) and let $s(z)$ be the associated function defined by (2.8). Then the following inequalities

$$
\left(\begin{array}{cc}
K & \Psi(z) \\
\Psi(z)^{*} & \frac{s(z)-s(z)^{*}}{z-\bar{z}}
\end{array}\right) \geq 0, \quad\left(\begin{array}{cc}
\widetilde{K} & z B \Psi(z)+\Pi_{1} \\
\bar{z} \Psi(z)^{*} B^{*}+\Pi_{1}^{*} & \frac{z s(z)-\bar{z} s(z)^{*}}{z-\bar{z}}
\end{array}\right) \geq 0
$$

hold for all $z \in \mathbb{C} \backslash(\mathbb{R} \cup \mathcal{Z})$ where

$$
\Psi(z)=\Gamma(z)\left\{\Pi_{2} s(z)+C \Pi_{1}\right\}
$$

and $\mathcal{Z}$ is a set defined via (1.1). 
Proof: Taking into account (1.4), we begin with the following evident inequalities

$$
\begin{aligned}
& \left(\begin{array}{cc}
K_{\sigma} & T \\
T^{*} & D
\end{array}\right)=\int_{\mathbb{R}_{+} \backslash \Lambda}\left(\begin{array}{c}
\Gamma(\lambda) \Pi_{2} \\
\frac{1}{\lambda-\bar{z}} I_{\mathcal{G}}
\end{array}\right) d \sigma(\lambda)\left(\Pi_{2}^{*} \Gamma(\lambda)^{*}, \frac{1}{\lambda-z} I_{\mathcal{G}}\right) \geq 0 \\
& \left(\begin{array}{cc}
\widetilde{K}_{\sigma} & \widetilde{T} \\
\widetilde{T}^{*} & \widetilde{D}
\end{array}\right)=\int_{\mathbb{R}_{+\backslash \Lambda}}\left(\begin{array}{c}
B \Gamma(\lambda) \Pi_{2} \\
\frac{1}{\lambda-\bar{z}} I_{\mathcal{G}}
\end{array}\right) \lambda d \sigma(\lambda)\left(\Pi_{2}^{*} \Gamma(\lambda)^{*} B^{*}, \frac{1}{\lambda-z} I_{\mathcal{G}}\right) \geq 0 .
\end{aligned}
$$

In view of (2.9) and (2.10),

$$
D=\frac{s(z)-s(z)^{*}}{z-\bar{z}}-\sum_{\lambda_{i} \in \Lambda} \frac{x_{i}}{\left|\lambda_{i}-z\right|^{2}}, \quad \widetilde{D}=\frac{z s(z)-\bar{z} s(z)^{*}}{z-\bar{z}}-\gamma-\sum_{\lambda_{i} \in \Lambda} \frac{\lambda_{i} x_{i}}{\left|\lambda_{i}-z\right|^{2}} .
$$

Using the resolvent like identity

$$
\Gamma(\lambda)-\Gamma(z)=(\lambda-z) \Gamma(\lambda) C B \Gamma(z) \quad(\lambda, z \in \mathbb{C} \backslash \mathcal{Z})
$$

which follows immediately from (1.2) and taking into account (1.6), (1.10), (2.8) and (2.12) we conclude from (2.11) that

$$
\begin{aligned}
T & =\int_{\mathbb{R}_{+} \backslash \Lambda} \Gamma(\lambda) \Pi_{2} \frac{d \sigma(\lambda)}{\lambda-z} \\
& =\Gamma(z) \Pi_{2} \int_{\mathbb{R}_{+} \backslash \Lambda} \frac{d \sigma(\lambda)}{\lambda-z}+\int_{\mathbb{R}_{+} \backslash \Lambda} \frac{\Gamma(\lambda)-\Gamma(z)}{\lambda-z} \Pi_{2} d \sigma(\lambda) \\
& =\Gamma(z) \Pi_{2}\left(s(z)-\gamma-\sum_{\lambda_{i} \in \Lambda} \frac{x_{i}}{\lambda_{i}-z}\right)+\Gamma(z) C \Pi_{1, \sigma} \\
& =\Psi(z)+\Gamma(z)\left\{C \Pi_{1, \sigma}-C \Pi_{1}-\Pi_{2} \gamma-\Pi_{2} \sum_{\lambda_{i} \in \Lambda} \frac{x_{i}}{\lambda_{i}-z}\right\} \\
& =\Psi(z)+\Gamma(z)\left\{C \widetilde{F} \gamma^{\frac{1}{2}}-\Pi_{2} \gamma-\sum_{\lambda_{i} \in \Lambda}\left(\frac{\Pi_{2} x_{i}}{\lambda_{i}-z}+C B F_{i} x_{i}^{\frac{1}{2}}\right)\right\}
\end{aligned}
$$

In view of the third condition in (1.11), $C \widetilde{F} \gamma^{\frac{1}{2}}-\Pi_{2} \gamma=0$ and using the second condition in (1.12) we obtain

$$
\begin{aligned}
\frac{1}{\lambda_{i}-z} \Pi_{2} x_{i}+C B F_{i} x_{i}^{\frac{1}{2}} & =\frac{1}{\lambda_{i}-z}\left\{\Pi_{2} x_{i}-\left(A-\lambda_{i} C B\right) F_{i} x_{i}^{\frac{1}{2}}+(A-z C B) F_{i} x_{i}^{\frac{1}{2}}\right\} \\
& =\frac{1}{\lambda_{i}-z}(A-z C B) F_{i} x_{i}^{\frac{1}{2}}
\end{aligned}
$$

which being substituted into (2.17) gives

$$
T=\Psi(z)-\sum_{\lambda_{i} \in \Lambda} \frac{F_{i} x_{i}^{\frac{1}{2}}}{\lambda_{i}-z}
$$

Similarly,

$$
\widetilde{T}:=\int_{\mathbb{R}_{+} \backslash \Lambda} B \Gamma(\lambda) \Pi_{2} \frac{\lambda d \sigma(\lambda)}{\lambda-z}
$$




$$
\begin{aligned}
& =z B \int_{\mathbb{R}_{+} \backslash \Lambda} \Gamma(\lambda) \Pi_{2} \frac{d \sigma(\lambda)}{\lambda-z}+\int_{\mathbb{R}_{+} \backslash \Lambda} B \Gamma(\lambda) \Pi_{2} d \sigma(\lambda) \\
& =z B \Psi(z)-z \sum_{\lambda_{i} \in \Lambda} \frac{B F_{i} x_{i}^{\frac{1}{2}}}{\lambda_{i}-z}+\Pi_{1, \sigma} \\
& =z B \Psi(z)+\Pi_{1}+\widetilde{F} \gamma^{\frac{1}{2}}-\sum_{\lambda_{i} \in \Lambda} \frac{\lambda_{i}}{\lambda_{i}-z} B F_{i} x_{i}^{\frac{1}{2}} .
\end{aligned}
$$

Finally, on account of (2.13) and (2.14), the inequalities

$$
\begin{gathered}
\left(\begin{array}{cc}
K_{\sigma} & T \\
T^{*} & D
\end{array}\right)+\sum_{\lambda_{i} \in \Lambda}\left(\begin{array}{c}
F_{i} \\
\frac{x_{i}^{2}}{\lambda_{i}-\bar{z}}
\end{array}\right)\left(F_{i}^{*}, \frac{x_{i}^{\frac{1}{2}}}{\lambda_{i}-z}\right)+\left(\begin{array}{cc}
P_{\infty}+\sum P_{i} & 0 \\
0 & 0
\end{array}\right) \geq 0 \\
\left(\begin{array}{cc}
\widetilde{K}_{\sigma} & \widetilde{T} \\
\widetilde{T}^{*} & \widetilde{D}
\end{array}\right)+\sum_{\lambda_{i} \in \Lambda} \lambda_{i}\left(\begin{array}{c}
B F_{i} \\
\frac{x_{i}^{\frac{1}{2}}}{\lambda_{i}-\bar{z}}
\end{array}\right)\left(\begin{array}{c}
F_{i}^{*} B^{*}, \frac{x_{i}^{\frac{1}{2}}}{\lambda_{i}-z} \\
)
\end{array}\right)+\left(\begin{array}{cc}
\widetilde{F} \widetilde{F}^{*}+\tilde{P}_{\infty}+\sum_{\infty} \lambda_{i} B P_{i} B^{*} & -\widetilde{F} \gamma^{\frac{1}{2}} \\
-\gamma^{\frac{1}{2}} \widetilde{F}^{*} & \gamma
\end{array}\right) \geq 0 .
\end{gathered}
$$

are in force and coincide in view of (1.8), (2.15), (2.18) and (2.19), with the fundamental matrix inequalities (2.11).

In Section 4 we shall prove the converse statement to Theorem 2.5: that every function $s$ which is analytic in $\mathbb{C} \backslash \mathbb{R}_{+}$and satisfies inequalities (2.11) at every point $z \in \mathbb{C} \backslash(\mathbb{R} \cup \mathcal{Z})$, defines via (2.4) a (unique) measure $d \sigma$ which is a solution of Problem 1.1. Thus, there exists a one to one correspondence between the set of all solutions $d \sigma$ of Problem 1.1 and the set of all analytic solutions $s$ of the system (2.11).

On the other hand we may make two immediate conclusions about a function $s$ which is a solution of (2.11). The first is that $s$ is a Stieltjes function (by Theorem 2.2, since the right bottom blocks in the matrices in (2.11) are both positive semidefinite). We also conclude from the first inequality in (2.11) (since the diagonal blocks $K$ and $\frac{s(z)-s(z)^{*}}{z-\bar{z}}$ are bounded at every point $\left.z \in \mathbb{C} \backslash \mathbb{R}_{+}\right)$that the offdiagonal block $\Psi(z)$ is bounded at every point $z \in \mathbb{C} \backslash \mathbb{R}_{+}$. To provide $\Psi(z)$ to be bounded in a neighborhood of any point $z_{i} \in \mathcal{Z} \backslash \mathbb{R}_{+}, s$ has to meet definite interpolation conditions at $z_{i}$ which can be easily expressed in terms of $A, B, C, \Pi_{1}$ and $\Pi_{2}$. If $\Gamma(z)$ is analytic on $\mathbb{R}_{+}$(see e.g., the example in Section 8), then every Stieltjes function $s$ satisfying these interpolation conditions is a solution of the system (2.11). Therefore, Problem 1.1 is equivalent to (and may be considered as) an interpolation problem for Stieltjes functions.

In a more general case when $\Gamma(z)$ is not necessarily analytic on $\mathbb{R}_{+}$(or at infinity), Problem 1.1 is still equivalent to an interpolation problem, a part of interpolation conditions of which is given in terms of asymptotic equalities (for the power moment problem such an asymptotic condition is established in the theorem of H. Hamburger and R. Nevanlinna [1, Section 3.1]).

\section{Transformation of fundamental matrix inequalities}

To establish the equivalence of Problem 1.1 and the system (2.11), it remains to show that every solution $s$ of (2.11) generates a measure $d \sigma$ which is a solution of Problem 1.1. To do 
it we apply a special transformation to the fundamental inequalities. Such a method was applied in [13], [17], [19] for quite different interpolation problems (we also refer to [18] for a general view on this method). Here we adapt the ideas from the papers just mentioned to the Stieltjes case (i.e., to the case of two fundamental matrix inequalities). We introduce the operator-valued function

$$
S(z):=K B^{*} C^{*} \Gamma(\bar{z})^{*}+\Gamma(z)\left\{\Pi_{2} s(z)+C \Pi_{1}\right\} \Pi_{2}^{*} \Gamma(\bar{z})^{*}=\left\{K B^{*} C^{*}+\Psi(z)\right\} \Gamma(\bar{z})^{*}
$$

( $\Psi$ is given by (2.12)) and show that it belongs to the Stieltjes class $\mathcal{S}(\mathcal{H})$. Moreover, we prove that $s(z)$ satisfies the system $(2.11)$ if and only if $S(z)$ satisfies a pair of much simplier inequalities (3.4). Since $\Gamma(z)$ has singular points in $\mathbb{C} \backslash \mathbb{R}_{+}$, the analyticity of $S$ in the whole $\mathbb{C} \backslash \mathbb{R}_{+}$does not follow directly from (3.1). To establish its analyticity in $\mathbb{C} \backslash \mathbb{R}_{+}$we need the following auxiliary lemma.

Lemma 3.1 Let $\mathcal{H}$ be separable Hilbert space, let $\Omega$ be a countable closed subset of $\mathbb{C} \backslash \mathbb{R}_{+}$ and let $S$ be a $\{\mathcal{H} ; \mathcal{H}\}$-valued function analytic on $\mathbb{C} \backslash\left(\mathbb{R}_{+} \cup \Omega\right)$ and such that

$$
\frac{S(z)-S(z)^{*}}{z-\bar{z}} \geq 0, \quad \frac{z S(z)-\bar{z} S(z)^{*}}{z-\bar{z}} \geq 0 \quad\left(\forall z \in \mathbb{C} \backslash\left(\mathbb{R}_{+} \cup \Omega\right)\right) .
$$

Then all possible singularities of $S$ in $\mathbb{C} \backslash \mathbb{R}_{+}$are removable and $S$ belongs to $\mathcal{S}(\mathcal{H})$.

Proof: First we prove the assertion of lemma for a scalar function $S$ (i.e for $\mathcal{H}=\mathbb{C}$ ). It is easily seen that if $S$ has the nonnegative (or nonpositive) imaginary (or real) part in some neighborhood $\mathcal{U}_{z_{0}}$ of the isolated singular point $z_{0}$, then the singularity at $z_{0}$ is removable. Let $z_{0} \in \mathbb{C} \backslash \mathbb{R}_{+}$be an isolated singular point of $S$ which satisfies $(3.2)$. If $z_{0} \in \mathbb{C}_{+}\left(z_{0} \in \mathbb{C}_{-}\right)$, then the first inequality in (3.2) provides the imaginary part of $S$ to be nonnegative (respectively, nonpositive) in some neighborhood $\mathcal{U}_{z_{0}}$. Therefore $z_{0}$ is a removable singular point of $S$. The second inequality in (3.2) is equivalent to

$$
\frac{S(z)+S(z)^{*}}{2} \geq-\frac{z+\bar{z}}{2} \cdot \frac{S(z)-S(z)^{*}}{z-\bar{z}}
$$

and implies in particular, that $S$ has a nonnegative real part in the left half-plane. Let $z_{0} \in \mathbb{R}_{-}$be an isolated singular point of $S$. Then $S$ has the nonnegative real part in some neighborhood $\mathcal{U}_{z_{0}}$ and also in this case the singularity of $S$ at $z_{0}$ is removable. By the assumption of lemma, every isolated point of $\Omega$ is an isolated singular point of $S$. By the preceding arguments, $S$ can be continuously extended to all of these points. Let us assume that $\Omega$ contains points which are not removable singular points of $S$. The set of all such points we denote by $\Omega^{\prime}$. The set $\Omega^{\prime}$ is countable (as a subset of a countable set $\Omega$ ) and closed (as a complement of the open set $\mathbb{C} \backslash\left(\mathbb{R}_{+} \cup \Omega^{\prime}\right)$, the domain of analyticity of the function $S)$. Therefore $\Omega^{\prime}$ contains an isolated point $z_{0} \in \Omega^{\prime}$ which is an isolated singular point of $S$. By the previous arguments, $z_{0}$ is a removable singular point of $S$ and $z_{0} \notin \Omega^{\prime}$, by the definition of $\Omega^{\prime}$. The obtained contradiction shows that the set $\Omega^{\prime}$ is empty and $\Omega$ consists only of removable singular points of $S$.

Now let $S$ be an operator-valued function which takes values in $\{\mathcal{H} ; \mathcal{H}\}$ and is subject to 
(3.2). Then for every choice of $f \in \mathcal{H}$, the scalar function $s_{f, f}(z)=\langle S(z) f, f\rangle_{\mathcal{H}}$ satisfies (3.2) and by the preceding analysis, it is analytic in $\mathbb{C} \backslash \mathbb{R}_{+}$. Therefore the function

$$
s_{f, g}(z):=\langle S(z) f, g\rangle_{\mathcal{H}}=\sum_{\ell=0}^{3}\left\langle S(z)\left(f+i^{\ell} g\right), f+i^{\ell} g\right\rangle_{\mathcal{H}}
$$

is analytic in $\mathcal{E}$ for every choice of $f, g \in \mathcal{H}$. This means that $S$ is analytic in the weak sense (and therefore, in the strong sense as well) in $\mathbb{C} \backslash \mathbb{R}$.

Since the set $\Omega$ is countable, for every $z_{0} \in \Omega \cap\{z: \Im z \neq 0\}$, there exists a sequence $\left\{z_{j}\right\}$ of points from $(\mathbb{C} \backslash \mathbb{R}) \backslash \Omega$ which converges to $z_{0}$. Inequalities (3.1) are valid at every point $z_{j}$ and therefore, they are in force at every nonreal point $z_{0} \in \Omega$. So, $S$ satisfies inequalities (3.2) all over $\mathbb{C} \backslash \mathbb{R}$ and by Theorem $2.2, S \in \mathcal{S}(\mathcal{H})$.

Theorem 3.2 Let $s(z)$ be a solution of the system (2.11). Then the function $S(z)$ defined by (3.1) belongs to the Stieltjes class $\mathcal{S}(\mathcal{H})$ and satisfies the system

$$
\left(\begin{array}{cc}
K & S(z) \\
S(z)^{*} & \frac{S(z)-S(z)^{*}}{z-\bar{z}}
\end{array}\right) \geq 0, \quad \quad\left(\begin{array}{cc}
\widetilde{K} & z B S(z)+B K \\
\bar{z} S(z)^{*} B^{*}+K B^{*} & \frac{z S(z)-\bar{z} S(z)^{*}}{z-\bar{z}}
\end{array}\right) \geq 0
$$

for all $z \in \mathbb{C} \backslash \mathbb{R}_{+}$. If moreover, $\Pi_{2} f \neq 0$ whenever $f \neq 0$, then s satisfies (2.11) if and only if $S(z)$ is a solution of (3.4).

Proof: Let $s(z)$ be a solution of the system $(2.11)$ and let $z$ be a point from $\mathbb{C} \backslash(\mathbb{R} \cup \mathcal{Z})$. Multiplying the first and the second inequalities from (2.11) by the block-operators

$$
\left(\begin{array}{cc}
I_{\mathcal{H}} & 0 \\
\Gamma(\bar{z}) C B & \Gamma(\bar{z}) \Pi_{2}
\end{array}\right) \in\{\mathcal{H} \oplus \mathcal{H} ; \mathcal{H} \oplus \mathcal{H}\}
$$

and

$$
\left(\begin{array}{cc}
I_{\widetilde{\mathcal{H}}} & 0 \\
\Gamma(\bar{z}) C & \Gamma(\bar{z}) \Pi_{2}
\end{array}\right) \in\{\widetilde{\mathcal{H}} \oplus \mathcal{H} ; \widetilde{\mathcal{H}} \oplus \mathcal{H}\}
$$

respectively on the left and by their adjoints on the right we obtain

$$
\left(\begin{array}{cc}
K & S(z) \\
S(z)^{*} & D(z)
\end{array}\right) \geq 0, \quad\left(\begin{array}{cc}
\widetilde{K} & \widetilde{S}(z) \\
\widetilde{S}(z)^{*} & \widetilde{D}(z)
\end{array}\right) \geq 0
$$

where $S(z)$ is the function given by (3.1) and where

$$
\begin{gathered}
\widetilde{S}(z)=\left\{\widetilde{K} C^{*}+\Pi_{1} \Pi_{2}^{*}+z B \Psi(z) \Pi_{2}^{*}\right\} \Gamma(\bar{z})^{*}, \\
D(z)=\Gamma(\bar{z})\left\{C B K B^{*} C^{*}+C B \Psi(z) \Pi_{2}^{*}+\Pi_{2} \Psi(z)^{*} B^{*} C^{*}\right. \\
\left.+\Pi_{2} \frac{s(z)-s(z)^{*}}{z-\bar{z}} \Pi_{2}^{*}\right\} \Gamma(\bar{z})^{*}, \\
\widetilde{D}(z)=\Gamma(\bar{z})\left\{C \widetilde{K} C^{*}+z C B \Psi(z) \Pi_{2}^{*}+C \Pi_{1} \Pi_{2}^{*}+\bar{z} \Pi_{2} \Psi(z)^{*} B^{*} C^{*}+\Pi_{2} \Pi_{1}^{*} C^{*}\right. \\
\left.+\Pi_{2} \frac{z s(z)-\bar{z} s(z)^{*}}{z-\bar{z}} \Pi_{2}^{*}\right\} \Gamma(\bar{z})^{*} .
\end{gathered}
$$


To simplify (3.6)-(3.8) we first note that in view of (1.2), (2.12) and (3.1),

$$
z S(z)+K=z \Psi(z) \Pi_{2}^{*} \Gamma(\bar{z})^{*}+K\left(I_{\mathcal{H}}+z B^{*} C^{*} \Gamma(\bar{z})^{*}\right)=\left(z \Psi(z) \Pi_{2}^{*}+K A^{*}\right) \Gamma(\bar{z})^{*}
$$

and

$$
z C B \Psi(z) \Pi_{2}^{*}+C \Pi_{1} \Pi_{2}^{*}=A \Psi(z) \Pi_{2}^{*}-\Pi_{2} s(z) \Pi_{2}^{*} .
$$

Next, the identities

$$
\begin{aligned}
\Gamma(\eta)\left\{\Pi_{2} \Pi_{1}^{*} C^{*}-C \Pi_{1} \Pi_{2}^{*}\right\} \Gamma(\omega)^{*}= & K B^{*} C^{*} \Gamma(\omega)^{*}-\Gamma(\eta) C B K \\
& +(\eta-\bar{\omega}) \Gamma(\eta) C B K B^{*} C^{*} \Gamma(\omega)^{*}
\end{aligned}
$$

and

$$
\Gamma(\eta)\left\{\eta C \Pi_{1} \Pi_{2}^{*}-\bar{\omega} \Pi_{2} \Pi_{1}^{*} C^{*}\right\} \Gamma(\omega)^{*}=\Gamma(\eta) A K-K A^{*} \Gamma(\omega)^{*}-(\eta-\bar{\omega}) \Gamma(\eta) C \widetilde{K} C^{*} \Gamma(\omega)^{*}
$$

hold for every pair of points $\eta$ and $\omega$ at which $\Gamma(z)$ is analytic and can be easily verified with help of (1.2) and (1.13). Substituting (1.13) into (3.6) and taking into account (3.9) we get

$$
\widetilde{S}(z)=\left\{B K A^{*}+z B \Psi(z) \Pi_{2}^{*}\right\} \Gamma(\bar{z})^{*}=z B S(z)+B K .
$$

Furthermore, substituting (3.11) and (2.16) (with $\lambda=\bar{z}$ ) into (3.7) and taking into account (3.1) and (2.12), we obtain

$$
\begin{aligned}
(z-\bar{z}) D(z)= & K B^{*} C^{*} \Gamma(\bar{z})^{*}-\Gamma(\bar{z}) C B K+\Gamma(\bar{z})\left\{C \Pi_{1} \Pi_{2}^{*}-\Pi_{2} \Pi_{1}^{*} C^{*}\right\} \Gamma(\bar{z})^{*} \\
& +\Gamma(\bar{z}) \Pi_{2}\left(s(z)-s(z)^{*}\right) \Pi_{2}^{*} \Gamma(\bar{z})^{*}+(\Gamma(z)-\Gamma(\bar{z}))\left\{\Pi_{2} s(z)+C \Pi_{1}\right\} \Pi_{2}^{*} \Gamma(\bar{z})^{*} \\
& +\Gamma(\bar{z}) \Pi_{2}\left\{s(z)^{*} \Pi_{2}^{*}+\Pi_{1}^{*} C^{*}\right\}\left(\Gamma(\bar{z})^{*}-\Gamma(z)^{*}\right)+ \\
= & K B^{*} C^{*} \Gamma(\bar{z})^{*}-\Gamma(\bar{z}) C B K+\Psi(z) \Pi_{2}^{*} \Gamma(\bar{z})^{*}-\Gamma(\bar{z}) \Pi_{2} \Psi(z)^{*} \\
= & S(z)-S(z)^{*} .
\end{aligned}
$$

Similarly substituting (3.10), (3.12) into (3.8) and using (3.9), (2.12) and the resolvent like identity

$$
\lambda \Gamma(\lambda)-z \Gamma(z)=(\lambda-z) \Gamma(\lambda) A \Gamma(z),
$$

we get

$$
\begin{aligned}
(z-\bar{z}) \widetilde{D}(z)= & K A^{*} \Gamma(\bar{z})^{*}-\Gamma(\bar{z}) A K+\Gamma(\bar{z})\left\{\bar{z} C \Pi_{1} \Pi_{2}^{*}-z \Pi_{2} \Pi_{1}^{*} C^{*}\right\} \Gamma(\bar{z})^{*} \\
& +(z \Gamma(z)-\bar{z} \Gamma(\bar{z}))\left\{\Pi_{2} s(z)+C \Pi_{1}\right\} \Pi_{2}^{*} \Gamma(\bar{z})^{*} \\
& +\Gamma(\bar{z}) \Pi_{2}\left\{s(z)^{*} \Pi_{2}^{*}+\Pi_{1}^{*} C^{*}\right\}\left(\Gamma(\bar{z})^{*}-\Gamma(z)^{*}\right) \\
& +\Gamma(\bar{z}) \Pi_{2}\left\{\bar{z} s(z)-z s(z)^{*}\right\} \Pi_{2}^{*} \Gamma(\bar{z})^{*} \\
= & K A^{*} \Gamma(\bar{z})^{*}-\Gamma(\bar{z}) A K+z \Psi(z) \Pi_{2}^{*} \Gamma(\bar{z})^{*}-\bar{z} \Gamma(\bar{z}) \Pi_{2} \Psi(z)^{*} \\
= & z S(z)-\bar{z} S(z)^{*} .
\end{aligned}
$$

Thus,

$$
D(z)=\frac{S(z)-S(z)^{*}}{z-\bar{z}}, \quad \widetilde{D}(z)=\frac{z S(z)-\bar{z} S(z)^{*}}{z-\bar{z}}
$$


which being substituted together with (3.13) into (3.5), lead to (3.4). So, inequalities (3.4) hold for every $z \in \mathbb{C} \backslash(\mathbb{R} \cup \mathcal{Z})$. By Lemma 3.1, $S \in \mathcal{S}(\mathcal{H})$. Since the set $\mathcal{Z}$ is countable, by the arguments from the proof of Lemma 3.1 we conclude that inequalities (3.4) are valid at every $z \in \mathbb{C} \backslash \mathbb{R}$.

Conversely, let $S$ be of the form (3.1) and satisfy (3.4), let $\Pi_{2}$ be injective and let $z$ be a point from $\mathbb{C} \backslash(\mathbb{R} \cup \mathcal{Z})$. Multiplying the first and the second inequalities from (3.4) by the block-operators

$$
\left(\begin{array}{cc}
I_{\mathcal{H}} & 0 \\
-C B & (A-\bar{z} C B)
\end{array}\right) \quad \text { and } \quad\left(\begin{array}{cc}
I_{\widetilde{\mathcal{H}}} & 0 \\
-C & (A-\bar{z} C B)
\end{array}\right)
$$

respectively on the left and by their adjoints on the right we get

$$
\left(\begin{array}{cc}
K & \Psi(z) \Pi_{2}^{*} \\
\Pi_{2} \Psi(z)^{*} & \Pi_{2} \frac{s(z)-s(z)^{*}}{z-\bar{z}} \Pi_{2}^{*}
\end{array}\right) \geq 0, \quad\left(\begin{array}{cc}
\widetilde{K} & \left(z B \Psi(z)+\Pi_{1}\right) \Pi_{2}^{*} \\
\Pi_{2}\left(\bar{z} \Psi(z)^{*} B^{*}+\Pi_{1}^{*}\right) & \Pi_{2} \frac{z s(z)-\bar{z} s(z)^{*}}{z-\bar{z}} \Pi_{2}^{*}
\end{array}\right) \geq 0 .
$$

Using notations

$$
\mathbf{K}(z):=\left(\begin{array}{cc}
K & \Psi(z) \\
\Psi(z)^{*} & \frac{s(z)-s(z)^{*}}{z-\bar{z}}
\end{array}\right) \quad \text { and } \quad \widetilde{\mathbf{K}}(z):=\left(\begin{array}{cc}
\widetilde{K} & z B \Psi(z)+\Pi_{1} \\
\bar{z} \Psi(z)^{*} B^{*}+\Pi_{1}^{*} & \frac{z s(z)-\bar{z} s(z)^{*}}{z-\bar{z}}
\end{array}\right)
$$

we rewrite two last inequalities as

$$
\left(\begin{array}{cc}
I_{\mathcal{H}} & 0 \\
0 & \Pi_{2}
\end{array}\right) \mathbf{K}(z)\left(\begin{array}{cc}
I_{\mathcal{H}} & 0 \\
0 & \Pi_{2}^{*}
\end{array}\right) \geq 0, \quad\left(\begin{array}{cc}
I_{\widetilde{\mathcal{H}}} & 0 \\
0 & \Pi_{2}
\end{array}\right) \widetilde{\mathbf{K}}(z)\left(\begin{array}{cc}
I_{\widetilde{\mathcal{H}}} & 0 \\
0 & \Pi_{2}^{*}
\end{array}\right) \geq 0
$$

Therefore

$$
\langle\mathbf{K}(z) f, f\rangle_{\mathcal{H} \oplus \mathcal{G}} \geq 0, \quad\langle\widetilde{\mathbf{K}}(z) g, g\rangle_{\widetilde{\mathcal{H}} \oplus \mathcal{G}} \geq 0
$$

for every choice of $f \in \mathcal{H} \oplus \operatorname{Ran} \Pi_{2}^{*}$ and $g \in \widetilde{\mathcal{H}} \oplus \operatorname{Ran} \Pi_{2}^{*}$. Since $\Pi_{2}$ injective, $\overline{\operatorname{Ran}}_{2}^{*}=\mathcal{G}$ and therefore, the inequalities (3.17) hold on dense sets in $\mathcal{H} \oplus \mathcal{G}$ and $\widetilde{\mathcal{H}} \oplus \mathcal{G}$, respectively. Since $\mathbf{K}(z)$ and $\widetilde{\mathbf{K}}(z)$ are bounded operators (for every $z \in \mathbb{C} \backslash \mathbb{R}+$ at which $\Gamma$ is analytic), it follows from (3.17) that $\mathbf{K}(z) \geq 0$ and $\widetilde{\mathbf{K}}(z) \geq 0$ which proves (2.11).

Theorem 3.3 Let S satisfy the system (3.4). Then it admits a representation

$$
S(z)=\int_{0}^{\infty} \frac{d \Sigma(\lambda)}{\lambda-z}
$$

with a nondecreasing $\{\mathcal{H} ; \mathcal{H}\}$-valued function $\Sigma(\lambda)$ on $\mathbb{R}_{+}$such that

$$
\text { (i) } \int_{0}^{\infty} d \Sigma(\lambda) \leq K, \quad(i i) \int_{0}^{\infty} B d \Sigma(\lambda)=B K, \quad(i i i) \quad \int_{0}^{\infty} \lambda B d \Sigma(\lambda) B^{*} \leq \widetilde{K}
$$

The integral in (3.18) and two first integrals in (3.19) converge uniformly while the third integral in (3.19) converges in the weak sense. 
Proof: Multiplying the first inequality in (3.4) by the block operator $\left(\begin{array}{cc}I_{\mathcal{H}} & 0 \\ \frac{1}{2} I_{\mathcal{H}} & \bar{z} I_{\mathcal{H}}\end{array}\right)$ on the left, by its adjoint on the right and evaluating the resulting inequality at $z=i y$ we obtain

$$
\left(\begin{array}{cc}
K & \frac{1}{2} K+i y S(i y) \\
\frac{1}{2} K-i y S(i y)^{*} & \frac{1}{4} K
\end{array}\right) \geq 0,
$$

which implies in particular, that

$$
\sup _{y \geq 1}\|y S(i y)\|<\infty
$$

It follows from (3.4) that

$$
\frac{S(z)-S(z)^{*}}{z-\bar{z}} \geq 0 \quad \text { and } \quad \frac{z S(z)-\bar{z} S(z)^{*}}{z-\bar{z}} \geq 0 \quad \text { for } \Im z \neq 0
$$

and therefore, $S(z)$ is a Stieltjes function, by Theorem 2.2. By Theorem 2.4 and in view of (3.21), $S$ admits a representation (3.18) with a $\{\mathcal{H} ; \mathcal{H}\}$-valued measure $d \Sigma(\lambda) \geq 0$ such that the integral $\int_{0}^{\infty} d \Sigma$ converges uniformly and $\left\|\int_{0}^{\infty} d \Sigma(\lambda)\right\|<\infty$. Let $M>0$ be such that $\left\|\int_{M}^{\infty} d \Sigma(\lambda)\right\|<\varepsilon$. Using (3.18) evaluated at $z=i y$, we obtain

$$
\begin{aligned}
\left\|i y S(i y)+\int_{0}^{\infty} d \Sigma(\lambda)\right\| & =\left\|\int_{0}^{\infty} \frac{\lambda}{\lambda-i y} d \Sigma(\lambda)\right\| \\
& \leq\left\|\int_{0}^{M} \frac{\lambda}{\sqrt{\lambda^{2}+y^{2}}} d \Sigma(\lambda)\right\|+\left\|\int_{M}^{\infty} \frac{\lambda}{\sqrt{\lambda^{2}+y^{2}}} d \Sigma(\lambda)\right\| \\
& \leq \frac{M}{\sqrt{M^{2}+y^{2}}}\left\|\int_{0}^{M} d \Sigma\right\|+\varepsilon
\end{aligned}
$$

and therefore uniformly,

$$
\lim _{y \rightarrow+\infty}-i y S(i y)=\int_{0}^{\infty} d \Sigma(\lambda) .
$$

Taking advantage of (3.20) and (3.22) we get

$$
\begin{aligned}
K-\int_{0}^{\infty} d \Sigma(\lambda) & =K+\lim _{y \rightarrow+\infty} i y S(i y) \\
& =\lim _{y \rightarrow+\infty}\left(\begin{array}{cc}
\frac{1}{2} I & I
\end{array}\right)\left(\begin{array}{cc}
K & \frac{1}{2} K+i y S(i y) \\
* & \frac{1}{4} K
\end{array}\right)\left(\begin{array}{c}
\frac{1}{2} I \\
I
\end{array}\right) \geq 0,
\end{aligned}
$$

which implies the first inequality in (3.19). Evaluating the second inequality in (3.4) at $z=i y$ we get

$$
\left(\begin{array}{cc}
\widetilde{K} & i y B S(i y)+B K \\
-i y S(i y)^{*} B^{*}+K B^{*} & \frac{1}{2}\left(S(i y)+S(i y)^{*}\right)
\end{array}\right) \geq 0 .
$$

By (3.22), $\lim _{y \rightarrow+\infty} S(i y)=0$ which together with (3.23) implies $\lim _{y \rightarrow+\infty}\|i y B S(i y)+B K\|=0$. Comparing this last equality with (3.22) we obtain (3.19(ii)). 
To prove $(3.19(\mathrm{iii}))$ we multiply $(3.23)$ by the block operator $\left(I_{\widetilde{\mathcal{H}}},-i y B\right)$ on the left and by its adjoint on the right. This leads to the inequality $\frac{1}{2} y^{2} B\left(S(i y)+S(i y)^{*}\right) B^{*} \leq \widetilde{K}$ for $y \geq 1$. Substituting (3.18) evaluated at $z=i y$ into this last inequality we get

$$
\int_{0}^{\infty} \frac{\lambda y^{2}}{\lambda^{2}+y^{2}} B d \Sigma(\lambda) B^{*} \leq \widetilde{K} \quad(y \geq 1) .
$$

By Fatou's lemma, for every $f \in \widetilde{\mathcal{H}}$,

$$
\int_{0}^{\infty} \lim _{y \rightarrow+\infty}\left\langle\frac{\lambda y^{2}}{\lambda^{2}+y^{2}} d \Sigma(\lambda) B^{*} f, B^{*} f\right\rangle_{\mathcal{H}}=\int_{0}^{\infty}\left\langle\lambda d \Sigma(\lambda) B^{*} f, B^{*} f\right\rangle_{\mathcal{H}} \leq\langle\widetilde{K} f, f\rangle_{\widetilde{\mathcal{H}}}
$$

which completes the proof of theorem.

Lemma 3.4 Let $s \in \mathcal{S}(\mathcal{G})$ be a solution of the system (2.11), let $S \in \mathcal{S}(\mathcal{H})$ be the associated function given by (3.1) and let $d \sigma$ and $d \Sigma$ be the measures from their integral representations (2.2) and (3.18). Then

$$
\int_{a}^{b}(A-\lambda C B) d \Sigma(\lambda)\left(A^{*}-\lambda B^{*} C^{*}\right)=\int_{a}^{b} \Pi_{2} d \sigma(\lambda) \Pi_{2}^{*}
$$

for every segment $[a ; b] \subset \mathbb{R}_{+}$.

Proof: Let $T(z)$ be the $\{\mathcal{H} ; \mathcal{H}\}$-valued function defined by

$$
T(z)=\Pi_{2} s(z) \Pi_{2}^{*} .
$$

Since $s \in \mathcal{S}(\mathcal{G}), T$ belongs to $\mathcal{S}(\mathcal{H})$. By $(3.1), T$ also can be represented as

$$
T(z)=(A-z C B) S(z)\left(A^{*}-z B^{*} C^{*}\right)-(A-z C B) K B^{*} C^{*}-C \Pi_{1} \Pi_{2}^{*} .
$$

Substituting (2.2) into (3.25) we get

$$
T(z)=\Pi_{2} \gamma \Pi_{2}^{*}+\int_{0}^{\infty} \Pi_{2} \frac{d \sigma(\lambda)}{\lambda-z} \Pi_{2}^{*} .
$$

On the other hand, upon substituting (3.18) into (3.26) we come to

$$
T(z)=C\left(\widetilde{K}-\int_{0}^{\infty} \lambda B d \Sigma(\lambda) B^{*}\right) C^{*}+\int_{0}^{\infty} \frac{(A-\lambda C B) d \Sigma(\lambda)\left(A^{*}-\lambda B^{*} C^{*}\right)}{\lambda-z} .
$$

Indeed, using (3.18), (3.26) and the equality

$$
\begin{aligned}
(A-z C B) \frac{d \Sigma(\lambda)}{\lambda-z}\left(A^{*}-z B^{*} C^{*}\right)= & (A-\lambda C B) \frac{d \Sigma(\lambda)}{\lambda-z}\left(A^{*}-\lambda B^{*} C^{*}\right)+C B d \Sigma(\lambda) A^{*} \\
& +(A-z C B) d \Sigma(\lambda) B^{*} C^{*}-\lambda C B d \Sigma(\lambda) B^{*} C^{*},
\end{aligned}
$$

we obtain

$$
T(z)=\Phi+\int_{0}^{\infty} \frac{(A-\lambda C B) d \Sigma(\lambda)\left(A^{*}-\lambda B^{*} C^{*}\right)}{\lambda-z}
$$


where

$\Phi=C B \int_{0}^{\infty} d \Sigma(\lambda) A^{*}+(A-z C B)\left\{\int_{0}^{\infty} d \Sigma(\lambda)-K\right\} B^{*} C^{*}-C \int_{0}^{\infty} \lambda B d \Sigma(\lambda) B^{*} C^{*}-C \Pi_{1} \Pi_{2}^{*}$.

In view of $(3.19(\mathrm{ii}))$ and $(1.13)$,

$$
\Phi=C B K A^{*}-C \int_{0}^{\infty} \lambda B d \Sigma(\lambda) B^{*} C^{*}-C \Pi_{1} \Pi_{2}^{*}=C\left(\widetilde{K}-\int_{0}^{\infty} \lambda B d \Sigma(\lambda) B^{*}\right) C^{*}
$$

which being substituted into (3.29), gives (3.28). Comparing two different representations (3.27) and (3.28) of the same function $T$ we conclude by the uniqueness, that

$$
C\left(\widetilde{K}-\int_{0}^{\infty} \lambda B d \Sigma(\lambda) B^{*}\right) C^{*}=\Pi_{2} \gamma \Pi_{2}^{*}
$$

and that the representing measures $\Pi_{2} \frac{d \sigma(\lambda)}{\lambda-z} \Pi_{2}^{*}$ and $(A-\lambda C B) d \Sigma(\lambda)\left(A^{*}-\lambda B^{*} C^{*}\right)$ coincide. The latter is equivalent to $(3.24)$.

Corollary 3.5 If $A-\lambda C B$ is boundedly invertible for every $\lambda \in[a, b]$ (i.e. if $[a, b] \subset$ $\mathbb{R}_{+} \backslash \Lambda$ ), then

$$
\int_{a}^{b} d \Sigma(\lambda)=\int_{a}^{b}(A-\lambda C B)^{-1} \Pi_{2} d \sigma(\lambda) \Pi_{2}^{*}\left(A^{*}-\lambda B^{*} C^{*}\right)^{-1}=\int_{a}^{b} \Gamma(\lambda) \Pi_{2} d \sigma(\lambda) \Gamma(\lambda)^{*}
$$

Lemma 3.6 Let $T \in\{\widetilde{\mathcal{H}} ; \widetilde{\mathcal{H}}\}$ be nonnegative and let $D \in\{\widetilde{\mathcal{H}} ; \mathcal{H}\}$ and $G \in\{\mathcal{G} ; \mathcal{H}\}$ satisfy

$$
D T D^{*}=G G^{*} .
$$

Then there exists an operator $S \in\{\mathcal{H} ; \mathcal{G}\}$ such that

$$
D S=G \quad \text { and } \quad S S^{*} \leq T .
$$

Proof: Representing (3.32) as $D T^{\frac{1}{2}}\left(D T^{\frac{1}{2}}\right)^{*}=G G^{*}$ we conclude by R. G. Douglas theorem [7] that there exists a partial isometry $\tilde{S}: \widetilde{\mathcal{H}} \rightarrow \mathcal{G}$ such that

$$
\tilde{S} \tilde{S}^{*} \leq I_{\mathcal{G}} \quad \text { and } \quad D T^{\frac{1}{2}} \tilde{S}=G .
$$

Setting $S:=T^{\frac{1}{2}} \tilde{S}$, we deduce (3.33) from the two last relations.

In conclusion of this section we discuss the inequalities (3.19(i)) and (3.19(iii)). Although $\int_{0}^{\infty} d \Sigma(\lambda)$ and $\int_{0}^{\infty} \lambda B d \Sigma(\lambda) B^{*}$ are not equal to $K$ and $\widetilde{K}$ respectively, their differences can be simply described. 
Lemma 3.7 Let $d \Sigma(\lambda) \geq 0$ be a $\{\mathcal{H} ; \mathcal{H}\}$-valued measure from the integral representation (3.18) of the function $S(z)$. Then

$$
K=\int_{0}^{\infty} d \Sigma(\lambda)+P_{\infty}, \quad \widetilde{K}=\int_{0}^{\infty} \lambda B d \Sigma(\lambda) B^{*}+\widetilde{F} \widetilde{F}^{*}+\tilde{P}_{\infty},
$$

where $P_{\infty} \in\{\mathcal{H} ; \mathcal{H}\}, \widetilde{P}_{\infty} \in\{\widetilde{\mathcal{H}} ; \widetilde{\mathcal{H}}\}$ and $\widetilde{F} \in\{\mathcal{G} ; \widetilde{\mathcal{H}}\}$ are bounded operators satisfying conditions (1.11).

Proof: It follows from $(3.19(\mathrm{i}))$ that the operator $P_{\infty}:=K-\int_{0}^{\infty} d \Sigma(\lambda)$ is nonnegative, while (3.19(ii)) implies that

$$
0=B\left(K-\int_{0}^{\infty} d \Sigma(\lambda)\right)=B P_{\infty}
$$

Making use of (3.30) and applying Lemma 3.6 to the operators

$$
T=\widetilde{K}-\int_{0}^{\infty} \lambda B d \Sigma(\lambda) B^{*} \in\{\widetilde{\mathcal{H}} ; \widetilde{\mathcal{H}}\}, \quad D=C \in\{\widetilde{\mathcal{H}}, \mathcal{H}\}, \quad G=\Pi_{2} \gamma^{\frac{1}{2}} \in\{\mathcal{G} ; \mathcal{H}\}
$$

we conclude that there exists an operator $\widetilde{F} \in\{\mathcal{G} ; \widetilde{\mathcal{H}}\}$ such that

$$
C \widetilde{F}=\Pi_{2} \gamma^{\frac{1}{2}} \quad \text { and } \quad \widetilde{F} \widetilde{F}^{*} \leq \widetilde{K}-\int_{0}^{\infty} \lambda B d \Sigma(\lambda) B^{*} .
$$

The second relation in (3.35) means that the operator

$$
\widetilde{P}_{\infty}:=\widetilde{K}-\int_{0}^{\infty} \lambda B d \Sigma(\lambda) B^{*}-\widetilde{F} \widetilde{F}^{*}
$$

is nonnegative while the first one together with (3.30) implies that

$$
C \widetilde{P}_{\infty} C^{*}=C\left(\widetilde{K}-\int_{0}^{\infty} \lambda B d \Sigma(\lambda) B^{*}-\widetilde{F} \widetilde{F}^{*}\right) C^{*}=\Pi_{2} \gamma \Pi_{2}^{*}-\Pi_{2} \gamma \Pi_{2}^{*}=0
$$

and therefore, $C \widetilde{P}_{\infty}=0$.

\section{On solutions of fundamental matrix inequalities}

In this section we show that every solution $s(z)$ of the system (2.11) induces the unique measure $d \sigma(\lambda) \geq 0$ on $\mathbb{R}_{+} \backslash \Lambda$ which gives the representations (1.8)-(1.10).

Let $s(z)$ satisfy (2.11) and admit a representation (2.2). By Theorems 3.2 and 3.3, the $\{\mathcal{H} ; \mathcal{H}\}$-valued function $S(z)$ defined by (3.1) admits an integral representation (3.18) with a measure $d \Sigma(\lambda) \geq 0$ which is subject to the constraints (3.19). Let $\Lambda=\left\{\lambda_{i}\right\}$ be the set defined in (1.3) and let $x_{i} \in\{\mathcal{G} ; \mathcal{G}\}$ and $X_{i} \in\{\mathcal{H} ; \mathcal{H}\}$ be nonnegative operators defined by

$$
x_{i}=\sigma\left(\lambda_{i}+0\right)-\sigma\left(\lambda_{i}-0\right) \quad \text { and } \quad X_{i}=\Sigma\left(\lambda_{i}+0\right)-\Sigma\left(\lambda_{i}-0\right) .
$$

Setting in (3.24) $a \rightarrow \lambda_{i}-0$ and $b \rightarrow \lambda_{i}+0$ we get

$$
\left(A-\lambda_{i} C B\right) X_{i}\left(A-\lambda_{i} C B\right)^{*}=\Pi_{2} x_{i} \Pi_{2}^{*} .
$$


Applying Lemma 3.6 to the operators

$$
T=X_{i} \in\{\mathcal{H} ; \mathcal{H}\}, \quad D=\left(A-\lambda_{i} C B\right) \in\{\mathcal{H}, \mathcal{H}\}, \quad G=\Pi_{2} x_{i}^{\frac{1}{2}} \in\{\mathcal{G} ; \mathcal{H}\}
$$

we conclude that there exists $F_{i} \in\{\mathcal{G} ; \mathcal{H}\}$ such that

$$
\left(A-\lambda_{i} C B\right) F_{i}=\Pi_{2} x_{i}^{\frac{1}{2}} \quad \text { and } \quad F_{i} F_{i}^{*} \leq X_{i} .
$$

The second relation in (4.3) means that the operator

$$
P_{i}:=X_{i}-F_{i} F_{i}^{*}
$$

is nonnegative while the first relation in (4.3) together with (4.2) implies that

$$
\begin{aligned}
\left(A-\lambda_{i} C B\right) P_{i}\left(A-\lambda_{i} C B\right)^{*} & =\left(A-\lambda_{i} C B\right)\left(X_{i}-F_{i} F_{i}^{*}\right)\left(A-\lambda_{i} C B\right)^{*} \\
& =\Pi_{2} x_{i} \Pi_{2}^{*}-\Pi_{2} x_{i} \Pi_{2}^{*}=0 .
\end{aligned}
$$

Therefore, $\left(A-\lambda_{i} C B\right) P_{i}=0$. Using notations (4.1) we rewrite (3.34) as

$$
K=K^{\circ}+P_{\infty}+\sum_{\lambda_{i} \in \Lambda} X_{i}, \quad \widetilde{K}=\widetilde{K}^{\circ}+\widetilde{P}_{\infty}+\widetilde{F} \widetilde{F}^{*}+\sum_{\lambda_{i} \in \Lambda} \lambda_{i} B X_{i} B^{*} .
$$

where

$$
K^{\circ}=\int_{\mathbb{R}_{+} \backslash \Lambda} d \Sigma(\lambda), \quad \widetilde{K}^{\circ}=\int_{\mathbb{R}_{+} \backslash \Lambda} \lambda B d \Sigma(\lambda) B^{*}
$$

and $P_{\infty}, \widetilde{P}_{\infty}, \widetilde{F}$ are operators satisfying (1.11). By (4.2)-(4.4), $X_{i}$ can be represented as

$$
X_{i}=P_{i}+F_{i} F_{i}^{*}
$$

where $P_{i}$ and $F_{i}$ are operators satisfying (1.12). On account of (3.31),

$$
\int_{\delta} d \Sigma(\lambda)=\int_{\delta} \Gamma(\lambda) \Pi_{2} d \sigma(\lambda) \Pi_{2}^{*} \Gamma(\lambda)^{*} .
$$

for every borel subset $\delta \subset \mathbb{R}_{+} \backslash \Lambda$. Therefore,

$$
\begin{aligned}
& K^{\circ}=\int_{\mathbb{R}_{+} \backslash \Lambda} d \Sigma(\lambda)=\int_{\mathbb{R}_{+} \backslash \Lambda} \Gamma(\lambda) \Pi_{2} d \sigma(\lambda) \Pi_{2}^{*} \Gamma(\lambda)^{*}=K_{\sigma} \\
& \widetilde{K}^{\circ}=\int_{\mathbb{R}_{+} \backslash \Lambda} \lambda B d \Sigma(\lambda) B^{*}=\int_{\mathbb{R}_{+} \backslash \Lambda} \lambda B \Gamma(\lambda) \Pi_{2} d \sigma(\lambda) \Pi_{2}^{*} \Gamma(\lambda)^{*} B^{*}=\widetilde{K}_{\sigma} .
\end{aligned}
$$

Substituting (4.6)-(4.8) into (4.5) we get the representations (1.8) and (1.9).

Theorem 4.1 Let $s$ be a solution of the system (2.11). Then $s$ belongs to the Stieltjes class $\mathcal{S}(\mathcal{G})$, admits a representation (2.2) with a measure $d \sigma \geq 0$ which in turn, gives the representations (1.8) and (1.9). If moreover, $\Pi_{2} f \neq 0$ whenever $f \neq 0$, then $d \sigma$ gives also the representation (1.10). 
Proof: The first claim of theorem concerning the representations (1.8) and (1.9) has been proved just before. Assuming $\Pi_{2}$ to be injective we substitute (1.8) and (1.9) into (1.13) and use (1.2), (1.11) and (1.12):

$$
\begin{aligned}
\Pi_{1} \Pi_{2}^{*} & =B K A^{*}-\widetilde{K} C^{*} \\
& =\int_{\mathbb{R}_{+} \backslash \Lambda} B \Gamma(\lambda) \Pi_{2} d \sigma(\lambda) \Pi_{2}^{*}+B P_{\infty} A^{*}-\widetilde{P}_{\infty} C^{*}-\widetilde{F} \widetilde{F}^{*} C^{*}+\sum_{i} B F_{i} F_{i}^{*}\left(A-\lambda_{i} C B\right)^{*} \\
& =\left\{\int_{\mathbb{R}_{+} \backslash \Lambda} B \Gamma(\lambda) \Pi_{2} d \sigma(\lambda)-\widetilde{F} \gamma^{\frac{1}{2}}+\sum_{i} B F_{i} x_{i}^{\frac{1}{2}}\right\} \Pi_{2}^{*} .
\end{aligned}
$$

The latter means that

$$
\Pi_{1} f=\left\{\int_{\mathbb{R}_{+} \backslash \Lambda} B \Gamma(\lambda) \Pi_{2} d \sigma(\lambda)-\widetilde{F} \gamma^{\frac{1}{2}}+\sum_{i} B F_{i} x_{i}^{\frac{1}{2}}\right\} f
$$

for every $f \in \operatorname{Ran} \Pi_{2}^{*}$. Since $\Pi_{2}$ is injective, $\overline{\operatorname{Ran} \Pi_{2}^{*}}=\mathcal{G}$ and (4.10) implies (1.10).

\section{Existence of a solution in the semidefinite case}

In this section we prove the sufficiency part of Theorem 1.3: if $\Pi_{2}^{*}$ is surjective, then Problem

\section{1 has a solution.}

By Theorem 4.1, it suffices to show that there exists a solution $s$ of system (2.11). We use a suitable adaptation of the regularization method which was applied by V. Katsnelson in [16], [15] to continuous interpolation problems (see also [23]). Setting $\widetilde{K}_{\varepsilon}$ to be a strictly positive operator defined by

$$
\widetilde{K}_{\varepsilon}=\widetilde{K}+\varepsilon I_{\widetilde{\mathcal{H}}} \quad(\varepsilon>0),
$$

we show that the Stieltjes function

$$
s_{\varepsilon}(z):=\Pi_{1}^{*}\left(\widetilde{K}_{\varepsilon}-z B K B^{*}\right)^{-1} \Pi_{1}
$$

is bounded uniformly with respect to $\varepsilon$ on every compact subset $\Omega$ in $\mathbb{C} \backslash \mathbb{R}_{+}$. Then there exists a sequence $s_{\varepsilon_{j}}$ converging $\left(\right.$ as $\varepsilon_{j} \rightarrow 0$ ) uniformly on every compact subset of $\mathbb{C} \backslash \mathbb{R}_{+}$to an analytic function $s$ which turns to be a solution of system (2.11). We begin with some auxiliary estimates.

Lemma 5.1 Let $S \in \mathcal{S}(\mathcal{H})$ satisfy the inequality

$$
\left(\begin{array}{cc}
K & S(z) \\
S(z)^{*} & \frac{S(z)-S(z)^{*}}{z-\bar{z}}
\end{array}\right) \geq 0 \quad(z \in \mathbb{C} \backslash \mathbb{R}) .
$$

Then

$$
\|S(z)\| \leq \frac{2\|K\|}{|z-\bar{z}|}(\Im z \neq 0) \quad \text { and } \quad\|S(z)\| \leq \frac{2\|K\|}{|z+\bar{z}|}(\Re z<0) \text {. }
$$


Proof: Multiplying $(5.2)$ by $\left(\frac{2}{\bar{z}-z} I_{\mathcal{H}}, I_{\mathcal{H}}\right)$ on the left and by its adjoint on the right we obtain

$$
\frac{4 K}{|z-\bar{z}|^{2}}-\frac{S(z)-S(z)^{*}}{z-\bar{z}} \geq 0
$$

which together with (5.2) implies

$$
\left(\begin{array}{cc}
K & S(z) \\
S(z)^{*} & \frac{4}{|z-\bar{z}|^{2}} K
\end{array}\right) \geq 0
$$

Therefore,

$$
\left|\langle S(z) f, g\rangle_{\mathcal{H}}\right|^{2} \leq\langle K f, f\rangle_{\mathcal{H}} \cdot\left\langle\frac{4}{|z-\bar{z}|^{2}} K g, g\right\rangle_{\mathcal{H}} \quad(\forall f, g \in \mathcal{H})
$$

and taking supremum in this last inequality through all $\|f\|=\|g\|=1$ we get $\|S(z)\|^{2} \leq$ $\frac{4}{|z-\bar{z}|^{2}}\|K\|^{2}$ which is equivalent to the first inequality in (5.3).

As a Stieltjes function, $S$ satisfies inequality (3.3) which together with (5.2) implies

$$
\left(\begin{array}{cc}
K & S(z) \\
S(z)^{*} & -\frac{S(z)+S(z)^{*}}{z+\bar{z}}
\end{array}\right) \geq 0 \quad(\forall z: \Re z<0) .
$$

Multiplying the latter inequality by $\left(\frac{2}{\bar{z}+z} I_{\mathcal{H}}, I_{\mathcal{H}}\right)$ on the left and by its adjoint on the right we get

$$
\frac{4 K}{|z+\bar{z}|^{2}}+\frac{S(z)+S(z)^{*}}{z+\bar{z}} \geq 0
$$

which being substituted into (5.4) implies

$$
\left(\begin{array}{cc}
K & S(z) \\
S(z)^{*} & \frac{4}{|z+\bar{z}|^{2}} K
\end{array}\right) \geq 0
$$

The arguments used just before lead to $\|S(z)\|^{2} \leq \frac{4}{|z+\bar{z}|^{2}}\|K\|^{2}$ and complete the proof of lemma.

Lemma 5.2 Let $\widetilde{K}_{\varepsilon}$ be defined by (5.1). Then

$$
\left\|\left(\widetilde{K}_{\varepsilon}-z B K B^{*}\right)^{-1}\right\| \leq \begin{cases}\frac{|z|}{\varepsilon|z-\bar{z}|}, & \Im z \neq 0 \\ \frac{|z|}{\varepsilon|z+\bar{z}|}, & \Re z<0\end{cases}
$$

Proof: It holds for every nonnegative operator $\mathbf{K}$ that

$$
\left\|(z I-\mathbf{K})^{-1}\right\|=\frac{1}{\operatorname{dist}(z ; \operatorname{spec} \mathbf{K})} \leq \frac{1}{\operatorname{dist}\left(z ; \mathbb{R}_{+}\right)} \leq \begin{cases}\frac{1}{|z-\bar{z}|}, & \Im z \neq 0 \\ \frac{1}{|z+\bar{z}|}, & \Re z<0\end{cases}
$$

Applying (5.6) to $\mathbf{K}=\widetilde{K}_{\varepsilon}^{-\frac{1}{2}} B K B^{*} \widetilde{K}_{\varepsilon}^{-\frac{1}{2}}$ and replacing $z$ by $\frac{1}{z}$, we obtain

$$
\left\|\left(I-z \widetilde{K}_{\varepsilon}^{-\frac{1}{2}} B K B^{*} \widetilde{K}_{\varepsilon}^{-\frac{1}{2}}\right)^{-1}\right\|=\frac{1}{|z|}\left\|\left(\frac{1}{z} I-\widetilde{K}_{\varepsilon}^{-\frac{1}{2}} B K B^{*} \widetilde{K}_{\varepsilon}^{-\frac{1}{2}}\right)^{-1}\right\| \leq \begin{cases}\frac{|z|}{|z-\bar{z}|}, & \Im z \neq 0 \\ \frac{|z|}{|z+\bar{z}|}, & \Re z<0\end{cases}
$$


Setting in (5.6) $\mathbf{K}=\widetilde{K}$ and $z=-\varepsilon^{2}$ we get

$$
\left\|\widetilde{K}_{\varepsilon}^{-1}\right\|=\left\|\left(\widetilde{K}+\varepsilon^{2} I\right)^{-1}\right\| \leq \frac{1}{\varepsilon^{2}}
$$

and since $\widetilde{K}_{\varepsilon}$ is selfadjoint,

$$
\left\|\widetilde{K}_{\varepsilon}^{-\frac{1}{2}}\right\|=\left\|\widetilde{K}_{\varepsilon}^{-1}\right\|^{\frac{1}{2}} \leq \frac{1}{\sqrt{\varepsilon}} .
$$

Estimates (5.5) follow from the evident inequality

$$
\left\|\left(\widetilde{K}_{\varepsilon}-z B K B^{*}\right)^{-1}\right\| \leq\left\|\widetilde{K}_{\varepsilon}^{-\frac{1}{2}}\right\| \cdot\left\|\left(I-z \widetilde{K}_{\varepsilon}^{-\frac{1}{2}} B K B^{*} \widetilde{K}_{\varepsilon}^{-\frac{1}{2}}\right)^{-1}\right\| \cdot\left\|\widetilde{K}_{\varepsilon}^{-\frac{1}{2}}\right\|
$$

in view of (5.7) and (5.8).

Corollary 5.3 The function $\varepsilon\left(\widetilde{K}_{\varepsilon}-z B K B^{*}\right)^{-1}$ is bounded uniformly with respect to $\varepsilon$ on every compact subset of $\mathbb{C} \backslash \mathbb{R}_{+}$.

Let us introduce the auxiliary space

$$
\widetilde{\mathcal{G}}=\mathcal{G} \oplus \mathcal{H}
$$

and operators

$$
\widetilde{\Pi}_{1, \varepsilon}=\left(\Pi_{1},-\varepsilon C^{*}\right) \in\{\widetilde{\mathcal{G}} ; \widetilde{\mathcal{H}}\}, \quad \widetilde{\Pi}_{2}=\left(\Pi_{2}, I_{\mathcal{H}}\right) \in\{\widetilde{\mathcal{G}} ; \mathcal{H}\}
$$

The regularized identity

$$
A K B^{*}-C \widetilde{K}_{\varepsilon}=\widetilde{\Pi}_{2} \widetilde{\Pi}_{1, \varepsilon}^{*}
$$

is a consequence of (1.13), (5.1) and (5.10). Let us consider the function

$$
\mathbf{S}_{\varepsilon}(z):=\widetilde{\Pi}_{1, \varepsilon}^{*}\left(\widetilde{K}_{\varepsilon}-z B K B^{*}\right)^{-1} \widetilde{\Pi}_{1, \varepsilon}
$$

which is $\{\widetilde{\mathcal{G}} ; \widetilde{\mathcal{G}}\}$-valued and analytic in $\mathbb{C} \backslash \mathbb{R}_{+}$in view of $(5.5)$.

Lemma 5.4 The function $\mathbf{S}_{\varepsilon}$ belongs to the Stieltjes class $\mathcal{S}(\widetilde{\mathcal{G}})$ and satisfies the regularized system

$$
\left(\begin{array}{cc}
K & \boldsymbol{\Psi}_{\varepsilon}(z) \\
\boldsymbol{\Psi}_{\varepsilon}(z)^{*} & \frac{\mathbf{S}_{\varepsilon}(z)-\mathbf{S}_{\varepsilon}(z)^{*}}{z-\bar{z}}
\end{array}\right) \geq 0, \quad\left(\begin{array}{cc}
\widetilde{K}_{\varepsilon} & z B \boldsymbol{\Psi}_{\varepsilon}(z)+\widetilde{\Pi}_{1, \varepsilon} \\
\bar{z} \boldsymbol{\Psi}_{\varepsilon}(z)^{*} B^{*}+\widetilde{\Pi}_{1, \varepsilon}^{*} & \frac{z \mathbf{S}_{\varepsilon}(z)-\bar{z} \mathbf{S}_{\varepsilon}(z)^{*}}{z-\bar{z}}
\end{array}\right) \geq 0
$$

for all $z \in \mathbb{C} \backslash(\mathbb{R} \cup \mathcal{Z})$ where $\mathcal{Z}$ is a set defined via (1.1) and

$$
\boldsymbol{\Psi}_{\varepsilon}(z)=\Gamma(z)\left\{\widetilde{\Pi}_{2} \mathbf{S}_{\varepsilon}(z)+C \widetilde{\Pi}_{1, \varepsilon}\right\} .
$$


Proof: It follows from (5.12) that

$$
\begin{aligned}
\frac{\mathbf{S}_{\varepsilon}(z)-\mathbf{S}_{\varepsilon}(z)^{*}}{z-\bar{z}} & =\widetilde{\Pi}_{1, \varepsilon}^{*}\left(\widetilde{K}_{\varepsilon}-\bar{z} B K B^{*}\right)^{-1} B K B^{*}\left(\widetilde{K}_{\varepsilon}-z B K B^{*}\right)^{-1} \widetilde{\Pi}_{1, \varepsilon} \\
\frac{z \mathbf{S}_{\varepsilon}(z)-\bar{z} \mathbf{S}_{\varepsilon}(z)^{*}}{z-\bar{z}} & =\widetilde{\Pi}_{1, \varepsilon}^{*}\left(\widetilde{K}_{\varepsilon}-\bar{z} B K B^{*}\right)^{-1} \widetilde{K}_{\varepsilon}\left(\widetilde{K}_{\varepsilon}-z B K B^{*}\right)^{-1} \widetilde{\Pi}_{1, \varepsilon} .
\end{aligned}
$$

Substituting (5.12) into (5.14) and using (1.2), (5.11) we get

$$
\begin{aligned}
\boldsymbol{\Psi}_{\varepsilon}(z) & =\Gamma(z)\left\{\widetilde{\Pi}_{2} \widetilde{\Pi}_{1, \varepsilon}^{*}+C\left(\widetilde{K}_{\varepsilon}-z B K B^{*}\right)\right\}\left(\widetilde{K}_{\varepsilon}-z B K B^{*}\right)^{-1} \widetilde{\Pi}_{1, \varepsilon}^{*} \\
& =\Gamma(z)\left\{A K B^{*}-z C B K B^{*}\right\}\left(\widetilde{K}_{\varepsilon}-z B K B^{*}\right)^{-1} \widetilde{\Pi}_{1, \varepsilon}^{*} \\
& =K B^{*}\left(\widetilde{K}_{\varepsilon}-z B K B^{*}\right)^{-1} \widetilde{\Pi}_{1, \varepsilon}^{*} .
\end{aligned}
$$

Therefore,

$$
\begin{aligned}
& z B \boldsymbol{\Psi}_{\varepsilon}(z)+\widetilde{\Pi}_{1, \varepsilon}^{*}=\left(I_{\widetilde{\mathcal{H}}}+z B K B^{*}\left(\widetilde{K}_{\varepsilon}-z B K B^{*}\right)^{-1}\right) \widetilde{\Pi}_{1, \varepsilon}^{*} \\
& =\widetilde{K}_{\varepsilon}\left(\widetilde{K}_{\varepsilon}-z B K B^{*}\right)^{-1} \widetilde{\Pi}_{1, \varepsilon}^{*} .
\end{aligned}
$$

Substituting (5.15)-(5.18) into (5.13) we get the following inequalities

$$
\begin{aligned}
& \left(\begin{array}{c}
\widetilde{\Pi}_{1, \varepsilon}^{*}\left(\widetilde{K}_{\varepsilon}-\bar{z} B K B^{*}\right)^{-1} B
\end{array}\right) K\left(I_{\mathcal{H}}, \quad B^{*}\left(\widetilde{K}_{\varepsilon}-z B K B^{*}\right)^{-1} \widetilde{\Pi}_{1, \varepsilon}\right) \geq 0 \\
& \left(\begin{array}{c}
I_{\widetilde{\mathcal{H}}} \\
\widetilde{\Pi}_{1, \varepsilon}^{*}\left(\widetilde{K}_{\varepsilon}-\bar{z} B K B^{*}\right)^{-1}
\end{array}\right) \widetilde{K}_{\varepsilon}\left(I_{\widetilde{\mathcal{H}}}, \quad\left(\widetilde{K}_{\varepsilon}-z B K B^{*}\right)^{-1} \widetilde{\Pi}_{1, \varepsilon}\right) \geq 0
\end{aligned}
$$

which are evidently true.

Lemma 5.5 Let $\mathbf{S}_{\varepsilon}$ be the function defined by (5.12) and let $\Omega$ be any compact set in $\mathbb{C} \backslash \mathbb{R}_{+}$. Then $\mathbf{S}_{\varepsilon}$ is bounded on $\Omega$ uniformly with respect to $\varepsilon$.

Proof: Using (5.10) we represent $\mathbf{S}_{\varepsilon}$ in the block form

$$
\mathbf{S}_{\varepsilon}(z)=\left(\begin{array}{cc}
s_{\varepsilon}(z) & s_{1, \varepsilon}(z) \\
s_{1, \varepsilon}(\bar{z})^{*} & s_{2, \varepsilon}(z)
\end{array}\right)=\left(\begin{array}{c}
\Pi_{1}^{*} \\
-\varepsilon C
\end{array}\right)\left(\widetilde{K}_{\varepsilon}-z B K B^{*}\right)^{-1}\left(\Pi_{1},-\varepsilon C^{*}\right)
$$

with respect to decomposition (5.9) of the space $\widetilde{\mathcal{G}}$ and conclude by Corollary 5.3, that the functions $s_{1, \varepsilon}$ and $s_{2, \varepsilon}$ are bounded in $\Omega$ uniformly with respect to $\varepsilon$. To establish the boundedness of $s_{\varepsilon}$ we apply Theorem 3.2 to the system (5.13) (this is possible since (5.13) has the same structure as (2.11) and since the identity (5.11) holds). By Theorem 3.2 we conclude in particular, that the function

$$
S(z):=K B^{*} C^{*} \Gamma(\bar{z})^{*}+\Gamma(z)\left\{\widetilde{\Pi}_{2} \mathbf{S}_{\varepsilon}(z)+C \widetilde{\Pi}_{1, \varepsilon}\right\} \widetilde{\Pi}_{2}^{*} \Gamma(\bar{z})^{*}
$$


belongs to the Stieltjes class $\mathcal{S}(\mathcal{H})$ and satisfies inequality (5.2) (or, that is the same, the first inequality in (3.4). By Lemma 5.1, $S$ is subject to estimates (5.3). By (5.20),

$$
\widetilde{\Pi}_{2} \mathbf{S}_{\varepsilon}(z) \widetilde{\Pi}_{2}^{*}=(A-z C B) S(z)\left(A^{*}-z B^{*} C^{*}\right)-(A-z C B) K B^{*} C^{*}-C \widetilde{\Pi}_{1, \varepsilon}
$$

and using (5.3) we conclude that the function $\widetilde{\Pi}_{2} \mathbf{S}_{\varepsilon}(z) \widetilde{\Pi}_{2}^{*}$ is bounded in $\Omega$ uniformly with respect to $\varepsilon$. In view of block representations (5.10), (5.19) of $\widetilde{\Pi}_{2}$ and $\mathbf{S}_{\varepsilon}$,

$$
\Pi_{2} s_{\varepsilon}(z) \Pi_{2}^{*}=\widetilde{\Pi}_{2} \mathbf{S}_{\varepsilon}(z) \widetilde{\Pi}_{2}^{*}+s_{1, \varepsilon}(\bar{z})^{*} \Pi_{2}^{*}+\Pi_{2} s_{1, \varepsilon}(z)-s_{2, \varepsilon}(z)
$$

and since all functions in the right hand side of the latter equality are bounded in $\Omega$ uniformly with respect to $\varepsilon$, the same conclusion is true for $\Pi_{2} s_{\varepsilon}(z) \Pi_{2}^{*}$. Since $\Pi_{2}^{*}$ is surjective (i.e., boundedly invertible from the right), the function $s_{\varepsilon}$ is bounded in $\Omega$ uniformly with respect to $\varepsilon$. Thus, all block entries in (5.19) are bounded which ends the proof of lemma.

Now we can complete the proof of Theorem 1.3. Take a sequence $\varepsilon_{j} \searrow+0$ and let $\mathbf{S}_{\varepsilon_{j}}(z)$ be Stieltjes functions defined via (5.19) with $\varepsilon=\varepsilon_{j}$. By Lemma 5.5, the family of functions $\mathbf{S}_{\varepsilon_{j}}(z)$ analytic in $\mathbb{C} \backslash \mathbb{R}_{+}$is bounded on each compact set in $\mathbb{C} \backslash \mathbb{R}_{+}$. Then there exists a subsequence $\mathbf{S}_{\varepsilon_{k}}(z)$ from this family which converges to an analytic function $\mathbf{S}(z)$ on each compact set in $\mathbb{C} \backslash \mathbb{R}_{+}$:

$$
\mathbf{S}(z)=\left(\begin{array}{cc}
s(z) & s_{1}(z) \\
s_{1}(\bar{z})^{*} & s_{2}(z)
\end{array}\right)=\lim _{\varepsilon_{k} \rightarrow 0}\left(\begin{array}{cc}
s_{\varepsilon}(z) & s_{1, \varepsilon_{k}}(z) \\
s_{1, \varepsilon_{k}}(\bar{z})^{*} & s_{2, \varepsilon_{k}}(z)
\end{array}\right)
$$

As a limit of Stieltjes functions $S$ belongs to $\mathcal{S}(\widetilde{\mathcal{G}})$. By $(5.5)$ and $(5.19)$,

$$
\left\|s_{2, \varepsilon_{k}}\right\|=\varepsilon_{k}^{2}\left\|C\left(\widetilde{K}_{\varepsilon}-z B K B^{*}\right)^{-1} C^{*}\right\| \leq \varepsilon_{k}\|C\|^{2} \cdot \begin{cases}\frac{|z|}{|z-\bar{z}|}, & \Im z \neq 0 \\ \frac{|z|}{|z+\bar{z}|}, & \Re z<0\end{cases}
$$

and therefore,

$$
s_{2}(z)=\lim _{\varepsilon_{k} \rightarrow 0} s_{2, \varepsilon_{k}}(z)=0
$$

on each compact set (and therefore, everywhere) in $\mathbb{C} \backslash \mathbb{R}_{+}$. Since $\mathbf{S}$ is a Stieltjes function, the latter equality implies that $s_{1}(z) \equiv 0$ (for $z=x<0$ this follows from the nonnegativity of $S(x)$ and for all $z \in \mathbb{C} \backslash \mathbb{R}_{+}$by the uniqueness theorem). Returning to the block decomposition (5.21) we conclude that $\mathbf{S}(z)$ is of the form

$$
\mathbf{S}(z)=\left(\begin{array}{cc}
s(z) & 0 \\
0 & 0
\end{array}\right)
$$

Since $\mathbf{S}_{\varepsilon_{k}}(z)$ is a solution of regularized inequalities (5.13), the limit function $\mathbf{S}(z)$ satisfies the following limit inequalities

$$
\left(\begin{array}{cc}
K & \mathbf{\Psi}(z) \\
\mathbf{\Psi}(z)^{*} & \frac{\mathbf{S}(z)-\mathbf{S}(z)^{*}}{z-\bar{z}}
\end{array}\right) \geq 0, \quad\left(\begin{array}{cc}
\widetilde{K} & z B \boldsymbol{\Psi}(z)+\Pi_{1} \\
\bar{z} \mathbf{\Psi}(z)^{*} B^{*}+\Pi_{1}^{*} & \frac{z \mathbf{S}(z)-\bar{z} \mathbf{S}(z)^{*}}{z-\bar{z}}
\end{array}\right) \geq 0
$$


for all $z \in \mathbb{C} \backslash(\mathbb{R} \cup \mathcal{Z})$ where, in accordance to (5.14) and (5.10),

$$
\mathbf{\Psi}(z)=\lim _{\varepsilon_{k} \rightarrow 0} \boldsymbol{\Psi}_{\varepsilon_{k}}(z)=\Gamma(z)\left\{\left(\Pi_{2}, I_{\mathcal{H}}\right) \mathbf{S}(z)+C\left(\Pi_{1}, 0\right)\right\}
$$

Upon substituting (5.22) and (5.24) into (5.23) it is readily seen that the function

$$
s(z)=\left.\mathrm{P}_{\mathcal{G}} \mathbf{S}(z)\right|_{\mathcal{G}}=\lim _{\varepsilon_{k} \rightarrow 0} s_{\varepsilon_{k}}(z)
$$

satisfies the system (2.11). By Theorem 4.1 this means that Problem 1.1 has a solution.

\section{$6 \quad$ Multiplicative Stieltjes classes}

Now we turn to the description of all solutions of Problem 1.1 (or equivalently, of all functions $s$ satisfying the system (2.11). Below we give such a description in terms of a linear fractional transformation (see Theorem 7.3) under assumptions

$$
K^{-1} \in\{\mathcal{H} ; \mathcal{H}\}, \quad \widetilde{K}^{-1} \in\{\widetilde{\mathcal{H}} ; \widetilde{\mathcal{H}}\} \quad \text { and } \quad \operatorname{Ran} \Pi_{2}^{*}=\mathcal{G} .
$$

It turns out that the "matrix" of coefficients of the linear fractional transformation describing all solutions of Problem 1.1 (the resolvent matrix of the problem) is an operator-valued function from the multiplicative Stieltjes class $\mathbf{W}_{\pi}$ which was introduced in [12] (for matrixvalued case) and studied in [9], [11].

Definition 6.1 A function $\Theta(z)$ which is $\{\mathcal{G} \oplus \mathcal{G} ; \mathcal{G} \oplus \mathcal{G}\}$-valued and analytic for almost all $z \in \mathbb{C}$ is said to be in the class $\mathbf{W}_{\pi}(\mathcal{G})$ if

$$
\begin{gathered}
\frac{\Theta(z) J \Theta(z)^{*}-J}{i(\bar{z}-z)} \geq 0 \quad(z \in \mathbb{C} \backslash \mathbb{R}), \quad \Theta(z) J \Theta(z)^{*}=J \quad(z \in \mathbb{R}), \\
\Theta(x) J_{\pi} \Theta(x)^{*} \geq J_{\pi} \quad(x<0),
\end{gathered}
$$

where

$$
J=\left(\begin{array}{cc}
0 & i I_{\mathcal{G}} \\
-i I_{\mathcal{G}} & 0
\end{array}\right), \quad J_{\pi}=\left(\begin{array}{cc}
0 & I_{\mathcal{G}} \\
I_{\mathcal{G}} & 0
\end{array}\right),
$$

and is said to be in the class $\mathbf{W}(\mathcal{G})$ if it satisfies only the conditions (6.2).

Remark 6.2 In Definition 6.1 and in what follows by "almost all points $z$ of a domain $\mathcal{E}$ " we mean all $z \in \mathcal{E}$ except at most countable closed set of points. For convenience, this exceptional set will be sometimes indicated.

Remark 6.3 The second property in (6.2) ( $J$-unitarity of $\Theta \in \mathbf{W}(\mathcal{G})$ almost everywhere on $\mathbb{R}$ ) implies by the symmetry principle, that

$$
\Theta(z)^{-1}=J \Theta(\bar{z})^{*} J
$$

and in particular, that $\Theta(z)$ is boundedly invertible for almost all $z$. 
It is easily seen that classes $\mathbf{W}_{\pi}(\mathcal{G})$ and $\mathbf{W}(\mathcal{G})$ are closed under multiplication: if $\Theta_{1}$ and $\Theta_{2}$ belong to $\mathbf{W}_{\pi}(\mathcal{G})$ (respectively, to $\mathbf{W}(\mathcal{G})$ ) then $\Theta_{1} \Theta_{2}$ and $\Theta_{2} \Theta_{1}$ also belong to $\mathbf{W}_{\pi}(\mathcal{G})$ (respectively, to $\mathbf{W}(\mathcal{G})$ ). The following theorem establishes the link between classes $\mathbf{W}(\mathcal{G})$ and $\mathbf{W}_{\pi}(\mathcal{G})$ and exhibits in fact an equivalent definition of the class $\mathbf{W}_{\pi}(\mathcal{G})$.

Theorem 6.4 A function $\Theta$ belongs to $\mathbf{W}_{\pi}(\mathcal{G})$ if and only if

$$
\Theta \in \mathbf{W}(\mathcal{G}) \quad \text { and } \quad \widetilde{\Theta}(z)=P(z) \Theta(z) P(z)^{-1} \in \mathbf{W}(\mathcal{G}),
$$

where

$$
P(z)=\left(\begin{array}{cc}
z I_{\mathcal{G}} & 0 \\
0 & I_{\mathcal{G}}
\end{array}\right)
$$

For the finite dimensional case $(\operatorname{dim} \mathcal{G}<\infty)$ this result was established in [12]. The proof for $\operatorname{dim} \mathcal{G}=\infty$ relies on similar arguments and will be omitted.

In this section we construct a function $\Theta \in \mathbf{W}_{\pi}(\mathcal{G})$ which corresponds to the interpolation data (1.14) satisfying the operator identity (1.13) and conditions (6.1).

Lemma 6.5 Let $\mu<0$ be a point such that $\Gamma(\mu)=(A-\mu C B)^{-1} \in\{\mathcal{H} ; \mathcal{H}\}$ and let $D \in$ $\{\mathcal{H} ; \mathcal{H}\}$ and $\widetilde{D} \in\{\widetilde{\mathcal{H}} ; \widetilde{\mathcal{H}}\}$ be defined as

$$
D=\Gamma(\mu)^{*}\left\{K^{-1}-\mu B^{*} \widetilde{K}^{-1} B\right\} \Gamma(\mu), \quad \widetilde{D}=\left(\widetilde{K}-\mu B K B^{*}\right)^{-1}
$$

Then the operators

$$
N=\left(\begin{array}{cc}
I_{\mathcal{G}}-\mu \Pi_{1}^{*} \widetilde{D} \Pi_{1} \Pi_{2}^{*} D \Pi_{2} & \Pi_{1}^{*} \widetilde{D} \Pi_{1} \\
-\mu \Pi_{2}^{*} D \Pi_{2} & I_{\mathcal{G}}
\end{array}\right)
$$

and

$$
\widetilde{N}=\left(\begin{array}{cc}
I_{\mathcal{G}}-\mu \Pi_{1}^{*} \widetilde{D} \Pi_{1} \Pi_{2}^{*} D \Pi_{2} & \mu \Pi_{1}^{*} \widetilde{D} \Pi_{1} \\
-\Pi_{2}^{*} D \Pi_{2} & I_{\mathcal{G}}
\end{array}\right)
$$

are J-unitary

$$
N J N^{*}=\widetilde{N} J \widetilde{N}^{*}=J
$$

and the following equalities hold

$$
\begin{aligned}
& \Gamma(\mu)\left(\Pi_{2}, C \Pi_{1}\right) N=K\left(A^{*} D \Pi_{2}, \quad B^{*} \widetilde{D} \Pi_{1}\right), \\
& \left(B \Gamma(\mu) \Pi_{2}, \quad G(\mu) \Pi_{1}\right) \widetilde{N}=\widetilde{K}\left(C^{*} D \Pi_{2}, \widetilde{D} \Pi_{1}\right) \text {, }
\end{aligned}
$$

where $G(z)$ is a $\{\widetilde{\mathcal{H}} ; \widetilde{\mathcal{H}}\}$-valued function defined by

$$
G(z)=I_{\widetilde{\mathcal{H}}}+z B \Gamma(z) C=I_{\widetilde{\mathcal{H}}}+z B(A-z C B)^{-1} C
$$

and where $J$ is the signature operator given in (6.3). 
Proof: Equalities (6.10) follow immediately from (6.8) and (6.9). Moreover, it is easily seen that the operators $N$ and $\widetilde{N}$ are $J$-unitary if and only if $D$ and $\widetilde{D}$ are selfadjoint. The verification of (6.11) and (6.12) is based on the following equalities

$$
\begin{aligned}
\Gamma(\mu)\left(\Pi_{2} \Pi_{1}^{*} \widetilde{D}+C\right) & =K B^{*} \widetilde{D} \\
\Gamma(\mu)-\mu K B^{*} \widetilde{D} \Pi_{1} \Pi_{2}^{*} D & =K A^{*} D
\end{aligned}
$$

the proof of which we begin with. The first equality follows from (1.13), (6.7) and (1.2):

$$
\begin{aligned}
\Gamma(\mu)\left(\Pi_{2} \Pi_{1}^{*} \widetilde{D}+C\right) & =\Gamma(\mu)\left(\Pi_{2} \Pi_{1}^{*}+C\left(\widetilde{K}-\mu B K B^{*}\right)\right) \widetilde{D} \\
& =\Gamma(\mu)\left(A K B^{*}-\mu C B K B^{*}\right) \widetilde{D} \\
& =\Gamma(\mu)(A-\mu C B) K B^{*} \widetilde{D}=K B^{*} \widetilde{D} .
\end{aligned}
$$

To prove (6.15) we note the equality

$$
\left(V^{-1}-Y X^{-1} Z\right)^{-1}=V+V Y(X-Z V Y)^{-1} Z V
$$

which holds for every choice of operators $X, Y, Z$ and $V$ acting on appropriate spaces and such that $X, V$ and $X-Z V Y$ are invertible. Applying this latter equality to the operators

$$
V=K, \quad X=\widetilde{K}, \quad Y=\mu B^{*}, \quad Z=B
$$

and taking advantage of the definition (6.7) of $\widetilde{D}$ we get

$$
\left(K^{-1}-\mu B^{*} \widetilde{K}^{-1} B\right)^{-1}=K+\mu K B^{*} \widetilde{D} B K
$$

which is equivalent to

$$
K\left(I_{\mathcal{H}}+\mu B^{*} \widetilde{D} B K\right)\left(K^{-1}-\mu B^{*} \widetilde{K}^{-1} B\right)=I_{\mathcal{H}}
$$

Note that in view of (6.14),

$$
I_{\mathcal{H}}+\mu B^{*} \widetilde{D} B K=I_{\mathcal{H}}+\mu B^{*}\left(\widetilde{D} \Pi_{1} \Pi_{2}^{*}+C\right) \Gamma(\mu)^{*}=\left(A^{*}+\mu B^{*} \widetilde{D} \Pi_{1} \Pi_{2}^{*}\right) \Gamma(\mu)^{*} .
$$

Substituting (6.18) into (6.17) and multiplying both sides of $(6.17)$ by $\Gamma(\mu)$ on the right, we obtain

$$
K\left(A^{*}+\mu B^{*} \widetilde{D} \Pi_{1} \Pi_{2}^{*}\right) D=\Gamma(\mu),
$$

which is equivalent to (6.15). Using (6.8) and (6.14) we get

$$
\begin{aligned}
\Gamma(\mu)\left(\Pi_{2}, \quad C \Pi_{1}\right) N & =\Gamma(\mu)\left(\Pi_{2}-\mu\left(\Pi_{2} \Pi_{1}^{*} \widetilde{D}+C\right) \Pi_{1} \Pi_{2}^{*} D \Pi_{2}, \quad\left(\Pi_{2} \Pi_{1}^{*} \widetilde{D}+C\right) \Pi_{1}\right) \\
& =\left(\Gamma(\mu) \Pi_{2}-\mu K B^{*} \widetilde{D} \Pi_{1} \Pi_{2}^{*} D \Pi_{2}, \quad K B^{*} \widetilde{D} \Pi_{1}\right)
\end{aligned}
$$

which coincides with (6.11), on account of (6.15). Making use of (6.11), (6.13), (1.13) and

$$
\widetilde{N}=\left(\begin{array}{cc}
\mu I_{\mathcal{G}} & 0 \\
0 & I_{\mathcal{G}}
\end{array}\right) N\left(\begin{array}{cc}
\mu^{-1} I_{\mathcal{G}} & 0 \\
0 & I_{\mathcal{G}}
\end{array}\right),
$$


we obtain

$$
\begin{aligned}
\left(B \Gamma(\mu) \Pi_{2}, \quad G(\mu) \Pi_{1}\right) \widetilde{N} & =\left(0, \Pi_{1}\right) \widetilde{N}+\mu B \Gamma(\mu)\left(\Pi_{2}, \quad C \Pi_{1}\right) N\left(\begin{array}{cc}
\mu^{-1} I_{\mathcal{G}} & 0 \\
0 & I_{\mathcal{G}}
\end{array}\right) \\
& =\left(-\Pi_{1} \Pi_{2}^{*} D \Pi_{2}, \Pi_{1}\right)+\mu B K\left(A^{*} D \Pi_{2}, \quad B^{*} \widetilde{D} \Pi_{1}\right)\left(\begin{array}{cc}
\mu^{-1} I_{\mathcal{G}} & 0 \\
0 & I_{\mathcal{G}}
\end{array}\right) \\
& =\left(\left(-\Pi_{1} \Pi_{2}^{*}+\mu B K A^{*}\right) D \Pi_{2}, \quad\left(I_{\mathcal{H}^{*}}+\mu B K B^{*} \widetilde{D}\right) \Pi_{1}\right) \\
& =\widetilde{K}\left(C^{*} D \Pi_{2}, \widetilde{D} \Pi_{1}\right)
\end{aligned}
$$

which proves (6.12).

Lemma 6.6 Let $K, \widetilde{K}, \Pi_{1}$ and $\Pi_{2}$ satisfy (1.13) and (6.1) and let $\Gamma, P, N, \widetilde{N}$, $G$ be the operators defined by (1.2), (6.6), (6.8), (6.9) and (6.13) respectively. Then the functions

$$
\begin{aligned}
& \Theta(z)=\left\{I_{\mathcal{G} \oplus \mathcal{G}}+(z-\mu)\left(\begin{array}{c}
\Pi_{1}^{*} C^{*} \\
-\Pi_{2}^{*}
\end{array}\right) \Gamma(\bar{z})^{*} K^{-1} \Gamma(\mu)\left(\Pi_{2}, C \Pi_{1}\right)\right\} N \\
& \widetilde{\Theta}(z)=\left\{I_{\mathcal{G} \oplus \mathcal{G}}+(z-\mu)\left(\begin{array}{c}
\Pi_{1}^{*} G(\bar{z})^{*} \\
-\Pi_{2}^{*} \Gamma(\bar{z})^{*} B^{*}
\end{array}\right) \widetilde{K}^{-1}\left(B \Gamma(\mu) \Pi_{2}, G(\mu) \Pi_{1}\right)\right\} \widetilde{N}
\end{aligned}
$$

are of the class $\mathbf{W}(\mathcal{G})$ and satisfy the relation

$$
\widetilde{\Theta}(z) P(z)=P(z) \Theta(z) .
$$

For every pair of points $z$ and $\omega$ at which $\Theta$ and $\widetilde{\Theta}$ are analytic, it holds that

$$
\frac{\Theta(z) J \Theta(\omega)^{*}-J}{i(\bar{\omega}-z)}=\left(\begin{array}{c}
\Pi_{1}^{*} C^{*} \\
-\Pi_{2}^{*}
\end{array}\right) \Gamma(\bar{z})^{*} K^{-1} \Gamma(\bar{\omega})\left(C \Pi_{1},-\Pi_{2}\right)
$$

and

$$
\frac{\widetilde{\Theta}(z) J \widetilde{\Theta}(\omega)^{*}-J}{i(\bar{\omega}-z)}=\left(\begin{array}{c}
\Pi_{1}^{*} G(\bar{z})^{*} \\
-\Pi_{2}^{*} \Gamma(\bar{z})^{*} B^{*}
\end{array}\right) \widetilde{K}^{-1}\left(G(\bar{\omega}) \Pi_{1},-B \Gamma(\bar{\omega}) \Pi_{2}\right) .
$$

Proof: First we note that by formulas (6.19), (6.20), each singular point of $\Theta$ or $\widetilde{\Theta}$ belongs to the set $\mathcal{Z}^{*}$ of all singular points of the function $\Gamma(\bar{z})^{*}$. This set is symmetric with respect to the real axis to the countable closed set $\mathcal{Z}$ defined by (1.1) and therefore, it is also countable and closed.

Since the operator $N$ is $J$-unitary, it follows from (6.19) that

$$
\Theta(z) J \Theta(\omega)^{*}-J=i\left(\begin{array}{c}
\Pi_{1}^{*} C^{*} \\
-\Pi_{2}^{*}
\end{array}\right) \Gamma(\bar{z})^{*} K^{-1} \Gamma(\mu) L(z, \omega) \Gamma(\mu)^{*} K^{-1} \Gamma(\bar{\omega})\left(C \Pi_{1},-\Pi_{2}\right)
$$

where

$$
\begin{aligned}
L(z, \omega)= & (\mu-z) \Gamma(\bar{\omega})^{-1} K \Gamma(\mu)^{-*}+(\bar{\omega}-\mu) \Gamma(\mu)^{-1} K \Gamma(\bar{z})^{-*} \\
& +(z-\mu)(\bar{\omega}-\mu)\left(\Pi_{2} \Pi_{1}^{*} C^{*}-C \Pi_{1} \Pi_{2}^{*}\right) .
\end{aligned}
$$


Using (1.2) and the equality

$$
\Pi_{2} \Pi_{1}^{*} C^{*}-C \Pi_{1} \Pi_{2}^{*}=A K B^{*} C^{*}-C B K A^{*}
$$

which can be easily derived from (1.13), we simplify (6.25) as

$$
\begin{aligned}
L(z, \omega)= & (\mu-z)(A-\bar{\omega} C B) K\left(A^{*}-\mu B^{*} C^{*}\right)+(\bar{\omega}-\mu)(A-\mu C B) K\left(A^{*}-z B^{*} C^{*}\right) \\
& +(z-\mu)(\bar{\omega}-\mu)\left(A K B^{*} C^{*}-C B K A^{*}\right) \\
= & (\bar{\omega}-z)(A-\mu C B) K\left(A^{*}-\mu B^{*} C^{*}\right) \\
= & (\bar{\omega}-z) \Gamma(\mu)^{-1} K \Gamma(\mu)^{-*} .
\end{aligned}
$$

Substituting the last equality into (6.24) we get (6.22). To show (6.23) we begin with equalities

$$
B \Gamma(\mu) \Pi_{2} \Pi_{1}^{*} G(\mu)^{*}-G(\mu) \Pi_{1} \Pi_{2}^{*} \Gamma(\mu)^{*} B^{*}=\widetilde{K} C^{*} \Gamma(\mu)^{*} B^{*}-B \Gamma(\mu) C \widetilde{K}
$$

and

$$
\frac{z \Gamma(z)-\omega \Gamma(\omega)}{z-\omega} C=\Gamma(z) C G(\omega)
$$

which are easily checked with help of (3.11). Since $\widetilde{N}$ is $J$-unitary, it follows from (6.20) that

$$
\begin{aligned}
\widetilde{\Theta}(z) J \widetilde{\Theta}(\omega)^{*}-J= & i(\mu-z)\left(\begin{array}{c}
\Pi_{1}^{*} G(\bar{z})^{*} \\
-\Pi_{2}^{*} \Gamma(\bar{z})^{*} B^{*}
\end{array}\right) \widetilde{K}^{-1}\left(G(\mu) \Pi_{1},-B \Gamma(\mu) \Pi_{2}\right) \\
& +i(\bar{\omega}-\mu)\left(\begin{array}{c}
\Pi_{1}^{*} G(\mu)^{*} \\
-\Pi_{2}^{*} \Gamma(\mu)^{*} B^{*}
\end{array}\right) \widetilde{K}^{-1}\left(G(\bar{\omega}) \Pi_{1},-B \Gamma(\bar{\omega}) \Pi_{2}\right) \\
& +i(z-\mu)(\bar{\omega}-\mu)\left(\begin{array}{c}
\Pi_{1}^{*} G(\bar{z})^{*} \\
-\Pi_{2}^{*} \Gamma(\bar{z})^{*} B^{*}
\end{array}\right) \widetilde{K}^{-1}\left\{B \Gamma(\mu) \Pi_{2} \Pi_{1}^{*} G(\mu)^{*}\right. \\
& \left.-G(\mu) \Pi_{1} \Pi_{2}^{*} \Gamma(\mu)^{*} B^{*}\right\} \widetilde{K}^{-1}\left(G(\bar{\omega}) \Pi_{1},-B \Gamma(\bar{\omega}) \Pi_{2}\right) .
\end{aligned}
$$

In view of (6.26), (6.27) and (2.16), the third term in the right hand side of the latter equality is equal to

$$
\begin{gathered}
i(z-\mu)(\bar{\omega}-\mu)\left(\begin{array}{c}
\Pi_{1}^{*} G(\bar{z})^{*} \\
-\Pi_{2}^{*} \Gamma(\bar{z})^{*} B^{*}
\end{array}\right)\left\{C^{*} \Gamma(\mu)^{*} B^{*} \widetilde{K}^{-1}-\widetilde{K}^{-1} B \Gamma(\mu) C\right\} \\
\times\left(G(\bar{\omega}) \Pi_{1},-B \Gamma(\bar{\omega}) \Pi_{2}\right) \\
=i(\bar{\omega}-\mu)\left(\begin{array}{c}
\Pi_{1}^{*} C^{*}\left(z \Gamma(\bar{z})^{*}-\mu \Gamma(\mu)^{*}\right) \\
-\Pi_{2}^{*}\left(\Gamma(\mu)^{*}-\Gamma(\bar{z})^{*}\right)
\end{array}\right) B^{*} \widetilde{K}^{-1}\left(G(\bar{\omega}) \Pi_{1},-B \Gamma(\bar{\omega}) \Pi_{2}\right) \\
\quad-i(z-\mu)\left(\begin{array}{c}
\Pi_{1}^{*} G(\bar{z})^{*} \\
-\Pi_{2}^{*} \Gamma(\bar{z})^{*} B^{*}
\end{array}\right) \widetilde{K}^{-1} B\left((\bar{\omega} \Gamma(\bar{\omega})-\mu \Gamma(\mu)) C \Pi_{1},(\Gamma(\mu)-\Gamma(\bar{\omega})) \Pi_{2}\right),
\end{gathered}
$$

which being substituted into (6.28) implies

$$
\begin{aligned}
& \widetilde{\Theta}(z) J \widetilde{\Theta}(\omega)^{*}-J \\
& =i(\mu-z)\left(\begin{array}{c}
\Pi_{1}^{*} G(\bar{z})^{*} \\
-\Pi_{2}^{*} \Gamma(\bar{z})^{*} B^{*}
\end{array}\right) \widetilde{K}^{-1}\left((G(\mu)+B(\bar{\omega} \Gamma(\bar{\omega})-\mu \Gamma(\mu)) C) \Pi_{1},-B \Gamma(\bar{\omega}) \Pi_{2}\right) \\
& \quad+i(\bar{\omega}-\mu)\left(\begin{array}{c}
\Pi_{1}^{*}\left(G(\mu)^{*}+C^{*}\left(z \Gamma(\bar{z})^{*}-\mu \Gamma(\mu)^{*}\right) B^{*}\right) \\
-\Pi_{2}^{*} \Gamma(\bar{z})^{*} B^{*}
\end{array}\right) \widetilde{K}^{-1}\left(G(\bar{\omega}) \Pi_{1},-B \Gamma(\bar{\omega}) \Pi_{2}\right) .
\end{aligned}
$$


The latter equality is equivalent to (6.23) since

$$
G(\mu)+B(z \Gamma(z)-\mu \Gamma(\mu)) C=G(z) .
$$

The equalities (6.22) and (6.23) imply in particular, that the functions $\Theta$ and $\widetilde{\Theta}$ have $J-$ properties (6.2). By (6.19), (6.20), $\Theta$ and $\widetilde{\Theta}$ are analytic in $\mathbb{C}$ except at most the set $\mathcal{Z}^{*}$ of all singular points of the function $\Gamma(\bar{z})^{*}$ which is countable and closed. Therefore, $\Theta$ and $\widetilde{\Theta}$ belong to the class $\mathbf{W}(\mathcal{G})$ according to Definition 6.1 . To prove (6.21) we note that on account of (6.19) and (6.20), it is equivalent to

$$
\begin{aligned}
& \widetilde{N} P(z)+(z-\mu)\left(\begin{array}{c}
\Pi_{1}^{*} G(\bar{z})^{*} \\
-\Pi_{2}^{*} \Gamma(\bar{z})^{*} B^{*}
\end{array}\right) \widetilde{K}^{-1}\left(B \Gamma(\mu) \Pi_{2}, G(\mu) \Pi_{1}\right) \widetilde{N} P(z) \\
& =P(z) N+(z-\mu) P(z)\left(\begin{array}{c}
\Pi_{1}^{*} C^{*} \\
-\Pi_{2}^{*}
\end{array}\right) \Gamma(\bar{z})^{*} K^{-1} \Gamma(\mu)\left(\Pi_{2}, C \Pi_{1}\right) N,
\end{aligned}
$$

which in turn, can be rewritten in view of (6.11) and (6.12), as

$$
\begin{aligned}
P(z) N-\widetilde{N} P(z)= & (z-\mu)\left(\begin{array}{c}
\Pi_{1}^{*} G(\bar{z})^{*} \\
-\Pi_{2}^{*} \Gamma(\bar{z})^{*} B^{*}
\end{array}\right)\left(C^{*} D \Pi_{2}, \widetilde{D} \Pi_{1}\right) P(z) \\
& -(z-\mu) P(z)\left(\begin{array}{c}
\Pi_{1}^{*} C^{*} \\
-\Pi_{2}^{*}
\end{array}\right) \Gamma(\bar{z})^{*}\left(A^{*} D \Pi_{2}, \quad B^{*} \widetilde{D} \Pi_{1}\right) .
\end{aligned}
$$

By (6.6), (6.8) and (6.9),

$$
P(z) N-\widetilde{N} P(z)=(z-\mu)\left(\begin{array}{cc}
0 & \Pi_{1}^{*} \widetilde{D} \Pi_{1} \\
\Pi_{2}^{*} D \Pi_{2} & 0
\end{array}\right)
$$

and therefore, (6.29) is equivalent to

$$
\left(\begin{array}{cc}
0 & \Pi_{1}^{*} \widetilde{D} \Pi_{1} \\
\Pi_{2}^{*} D \Pi_{2} & 0
\end{array}\right)=\left(\begin{array}{cc}
\Pi_{1}^{*} & 0 \\
0 & \Pi_{2}^{*}
\end{array}\right) \Upsilon(z)\left(\begin{array}{cc}
D \Pi_{2} & 0 \\
0 & \widetilde{D} \Pi_{1}
\end{array}\right)
$$

where

$$
\Upsilon(z)=\left(\begin{array}{cc}
z G(\bar{z})^{*} C^{*}-z C^{*} \Gamma(\bar{z})^{*} A^{*} & G(\bar{z})^{*}-z C^{*} \Gamma(\bar{z})^{*} B^{*} \\
\Gamma(\bar{z})^{*} A^{*}-z \Gamma(\bar{z})^{*} B^{*} C^{*} & 0
\end{array}\right)
$$

It follows immediately from (1.2) and (6.13) that $\Upsilon(z)=\left(\begin{array}{cc}0 & I_{\widetilde{\mathcal{H}}} \\ I_{\mathcal{H}} & 0\end{array}\right)$ and thus, (6.30) (which is equivalent to $(6.21))$ is in force.

Remark 6.7 Since both $\Theta$ and $\widetilde{\Theta}$ belong to $\mathbf{W}(\mathcal{G})$, it follows from (6.21) by Theorem 6.4 that $\Theta$ is of the class $\mathbf{W}_{\pi}(\mathcal{G})$.

Corollary 6.8 Let $\Theta$ and $\widetilde{\Theta}$ be defined by (6.19) and (6.20) respectively. It holds that for every choice of points $z$ and $\omega$ at which $\Theta^{-1}$ and $\widetilde{\Theta}^{-1}$ are analytic,

$$
\frac{J-\Theta(\omega)^{-*} J \Theta(z)^{-1}}{i(\bar{\omega}-z)}=\left(\begin{array}{l}
\Pi_{2}^{*} \\
\Pi_{1}^{*} C^{*}
\end{array}\right) \Gamma(\omega)^{*} K^{-1} \Gamma(z)\left(\Pi_{2}, C \Pi_{1}\right)
$$


and

$$
\begin{aligned}
\frac{J-\widetilde{\Theta}(\omega)^{-*} J \widetilde{\Theta}(z)^{-1}}{i(\bar{\omega}-z)} & =\frac{J-P(\omega)^{-*} \Theta(\omega)^{-*} P(\omega)^{*} J P(z) \Theta(z)^{-1} P^{-1}(z)}{i(\bar{\omega}-z)} \\
& =\left(\begin{array}{c}
\Pi_{2}^{*} \Gamma(\omega)^{*} B^{*} \\
\Pi_{1}^{*} G(\omega)^{*}
\end{array}\right) \widetilde{K}^{-1}\left(B \Gamma(z) \Pi_{2}, G(z) \Pi_{1}\right) .
\end{aligned}
$$

Proof: The first equality in (6.32) is a consequence of (6.21). Further, the symmetry relation (6.4) for $\Theta$ and a similar relation for $\widetilde{\Theta}$ imply that

$$
\begin{aligned}
& \Theta(\omega)^{-*} J \Theta(z)^{-1}-J=J\left(\Theta(\bar{\omega}) J \Theta(\bar{z})^{*}-J\right) J \\
& \widetilde{\Theta}(\omega)^{-*} J \widetilde{\Theta}(z)^{-1}-J=J\left(\widetilde{\Theta}(\bar{\omega}) J \widetilde{\Theta}(\bar{z})^{*}-J\right) J .
\end{aligned}
$$

Substituting (6.22) and (6.23) into right hand sides in the two latter equalities and taking into account the structure (6.3) of the operator $J$ we derive (6.31) and (6.32).

It will be shown in the next section that the function $\Theta$ constructed via (6.19) is the matrix of coefficients of the linear fractional transformation describing all solutions of Problem 1.1. The formula (6.19) for $\Theta$ can be essentially simplified when the operator $A$ from (1.14) is the identity operator. This is the case for a number of concrete interpolation problems (e.g., the Stieltjes moment problem, continuous interpolation problems on the halfaxis; see Sections 10 and 11). For such a choice of $A$, the functions $\Gamma(z)$ and $G(z)$ defined via (1.2) and (6.13), are of the form

$$
\begin{aligned}
\Gamma(z) & =\left(I_{\mathcal{H}}-z C B\right)^{-1} \\
G(z) & =I_{\widetilde{\mathcal{H}}}+z B\left(I_{\mathcal{H}}-z C B\right)^{-1} C=\left(I_{\widetilde{\mathcal{H}}}-z B C\right)^{-1}
\end{aligned}
$$

and Lemma 6.6 can be reformulated as

Lemma 6.9 Let $K, \widetilde{K}$ be strictly positive operators satisfying the identity

$$
K B^{*}-C \widetilde{K}=\Pi_{2} \Pi_{1}^{*},
$$

and let $N, \widetilde{N}$ be $J$-unitary operators defined by

$$
N=\left(\begin{array}{cc}
I_{\mathcal{G}} & \Pi_{1}^{*} \widetilde{K}^{-1} \Pi_{1} \\
0 & I_{\mathcal{G}}
\end{array}\right), \quad \widetilde{N}=\left(\begin{array}{cc}
I_{\mathcal{G}} & 0 \\
-\Pi_{2}^{*} K^{-1} \Pi_{2} & I_{\mathcal{G}}
\end{array}\right) .
$$

Then the functions

$$
\begin{aligned}
& \Theta(z)=\left\{I_{\mathcal{G} \oplus \mathcal{G}}+z\left(\begin{array}{c}
\Pi_{1}^{*} C^{*} \\
-\Pi_{2}^{*}
\end{array}\right)\left(I_{\mathcal{H}}-z B^{*} C^{*}\right)^{-1} K^{-1}\left(\Pi_{2}, C \Pi_{1}\right)\right\} N \\
& \widetilde{\Theta}(z)=\left\{I_{\mathcal{G} \oplus \mathcal{G}}+z\left(\begin{array}{c}
\Pi_{1}^{*} \\
-\Pi_{2}^{*} B^{*}
\end{array}\right)\left(I_{\widetilde{\mathcal{H}}}-z C^{*} B^{*}\right)^{-1} \widetilde{K}^{-1}\left(B \Pi_{2}, \Pi_{1}\right)\right\} \widetilde{N}
\end{aligned}
$$

are of the class $\mathbf{W}(\mathcal{G})$ and satisfy the relation (6.21). In particular, $\Theta$ belongs to $\mathbf{W}_{\pi}(\mathcal{G})$.

Proof: Since $\Gamma(z)$ is analytic at the origin, one can set $\mu=0$ in Lemmas 6.5 and 6.6. Since $\Gamma(0)=I_{\mathcal{H}}$, it follows from (6.7) that $D=K^{-1}, \widetilde{D}=\widetilde{K}^{-1}$ and thus, the operators $N$ and $\widetilde{N}$ defined via (6.8) and (6.9), respectively, becomes to (6.34). Now the formulas (6.35) and (6.36) are immediate consequences of (6.19) and (6.20), respectively and all assertions of the lemma follow from Lemma 6.6. 


\section{Description of all solutions}

In this section we parametrize all solutions of Problem 1.1 by a linear fractional transformation. To formulate the corresponding result we need a definition of a Stieltjes pair which we now recall.

Definition 7.1 A pair $\{p(z), q(z)\}$ of two $\{\mathcal{G} ; \mathcal{G}\}$-valued functions $p$ and $q$ analytic almost everywhere in $\mathbb{C} \backslash \mathbb{R}_{+}$is called a Stieltjes pair if

$$
p(z)^{*} p(z)+q(z)^{*} q(z) \geq \varepsilon(z) I_{\mathcal{G}}
$$

for some $\varepsilon>0$ depending on $z$ and for almost all $z \in \mathbb{C} \backslash \mathbb{R}_{+}$(the nondegeneracy of the pair), and if the following inequalities

$$
\begin{aligned}
\left(p(z)^{*}, q(z)^{*}\right) \frac{J}{i(\bar{z}-z)}\left(\begin{array}{c}
p(z) \\
q(z)
\end{array}\right) & =\frac{q(z)^{*} p(z)-p(z)^{*} q(z)}{z-\bar{z}} \geq 0 \\
\left(p(z)^{*}, q(z)^{*}\right) \frac{P(z)^{*} J P(z)}{i(\bar{z}-z)}\left(\begin{array}{c}
p(z) \\
q(z)
\end{array}\right) & =\frac{z q(z)^{*} p(z)-\bar{z} p(z)^{*} q(z)}{z-\bar{z}} \geq 0
\end{aligned}
$$

hold for every $z \in \mathbb{C} \backslash \mathbb{R}$ where $p$ and $q$ are analytic.

Here $J$ and $P(z)$ are operators defined in (6.3) and (6.6) respectively. A pair $\{p, q\}$ is said to be equivalent to the pair $\left\{p_{1}, q_{1}\right\}$ if there exists a $\{\mathcal{G} ; \mathcal{G}\}$-valued meromorphic function $R(z)$ which is boundedly invertible (i.e. $R^{-1} \in\{\mathcal{G} ; \mathcal{G}\}$ ) at almost all points $z \in \mathbb{C} \backslash \mathbb{R}_{+}$and such that

$$
p_{1}(z)=p(z) R(z) \quad \text { and } \quad q_{1}(z)=q(z) R(z) .
$$

Lemma 7.2 Under hypothesis (6.1) the system (2.11) of the fundamental matrix inequalities is equivalent to the following system

$$
\begin{gathered}
\left(s(z)^{*}, I_{\mathcal{G}}\right) \frac{\Theta(z)^{-*} J \Theta^{-1}(z)}{i(\bar{z}-z)}\left(\begin{array}{c}
s(z) \\
I_{\mathcal{G}}
\end{array}\right) \geq 0 \\
\left(s(z)^{*}, I_{\mathcal{G}}\right) \frac{\Theta(z)^{-*} P(z)^{*} J P(z) \Theta^{-1}(z)}{i(\bar{z}-z)}\left(\begin{array}{c}
s(z) \\
I_{\mathcal{G}}
\end{array}\right) \geq 0, \quad(z \in \mathbb{C} \backslash(\mathbb{R} \cup \mathcal{Z}))
\end{gathered}
$$

where $\Theta$ is the function given by (6.19).

Proof: Since $K$ and $\widetilde{K}$ are boundedly invertible and positive, the system (2.11) is equivalent to

$$
\begin{aligned}
\frac{s(z)-s(z)^{*}}{z-\bar{z}}-\Psi(z)^{*} K^{-1} \Psi(z) & \geq 0 \\
\frac{z s(z)-\bar{z} s(z)^{*}}{z-\bar{z}}-\left(z B \Psi(z)+\Pi_{1}\right)^{*} \hat{K}^{-1}\left(z B \Psi(z)+\Pi_{1}\right) & \geq 0 .
\end{aligned}
$$

In view of (2.12), (6.6) and (6.13),

$$
\Psi(z)=\Gamma(z)\left(\Pi_{2}, C \Pi_{1}\right)\left(\begin{array}{c}
s(z) \\
I_{\mathcal{G}}
\end{array}\right), \quad z B \Psi(z)+\Pi_{1}=\left(B \Gamma(z) \Pi_{2}, G(z) \Pi_{1}\right) P(z)\left(\begin{array}{c}
s(z) \\
I_{\mathcal{G}}
\end{array}\right)
$$


which allows to rewrite (7.7) and (7.8) as

$$
\begin{array}{r}
\left(s(z)^{*}, I_{\mathcal{G}}\right)\left\{\frac{J}{i(\bar{z}-z)}-\left(\begin{array}{c}
\Pi_{2}^{*} \\
\Pi_{1}^{*} C^{*}
\end{array}\right) \Gamma(z)^{*} K^{-1} \Gamma(z)\left(\Pi_{2}, C \Pi_{1}\right)\right\}\left(\begin{array}{c}
s(z) \\
I_{\mathcal{G}}
\end{array}\right) \geq 0, \\
\left(s(z)^{*}, I_{\mathcal{G}}\right) P(z)^{*}\left\{\frac{J}{i(\bar{z}-z)}-\left(\begin{array}{c}
\Pi_{2}^{*} \Gamma(z)^{*} B^{*} \\
\Pi_{1}^{*} G(z)^{*}
\end{array}\right) \widetilde{K}^{-1}\left(B \Gamma(z) \Pi_{2}, G(z) \Pi_{1}\right)\right\} P(z)\left(\begin{array}{c}
s(z) \\
I_{\mathcal{G}}
\end{array}\right) \geq 0 .
\end{array}
$$

In view of (6.31) and (6.32), the two latter inequalities coincide with (7.5) and (7.6), respectively.

The next theorem describes all the solutions $s$ of the system of inequalities (7.5), (7.6) (or equivalently, of the system of the fundamental matrix inequalities (2.11)). By Theorems 2.5 and 4.1, every such $s$ is a Stieltjes function and the associated measure $d \sigma$ (uniquely defined by $s$ via the Stieltjes inversion formula (2.4)) is a solution of Problem 1.1. A system of such type for the finite dimensional case $(\operatorname{dim} \mathcal{G}<\infty)$ was considered in [11]. The corresponding arguments are applicable to the infinite dimensional situation; we present them for the sake of completeness.

Theorem 7.3 Let (6.1) be fulfilled and let

$$
\Theta(z)=\left(\begin{array}{cc}
\Theta_{11}(z) & \Theta_{12}(z) \\
\Theta_{21}(z) & \Theta_{22}(z)
\end{array}\right)
$$

be the block decomposition of the function $\Theta \in \mathbf{W}_{\pi}$ defined by (6.19) into four $\{\mathcal{G} ; \mathcal{G}\}-$ valued blocks. Then the function s satisfies inequalities (7.5), (7.6) if and only if it can be represented in the form

$$
s(z)=\left(\Theta_{11}(z) p(z)+\Theta_{12}(z) q(z)\right)\left(\Theta_{21}(z) p(z)+\Theta_{22}(z) q(z)\right)^{-1}
$$

for some Stieltjes pair $\{p, q\} \in \overline{\mathcal{S}}(\mathcal{G})$ such that

$$
\left(\Theta_{21}(z) p(z)+\Theta_{22}(z) q(z)\right)^{-1} \in\{\mathcal{G} ; \mathcal{G}\} \quad\left(\forall z \in \mathbb{C} \backslash \mathbb{R}_{+}\right) .
$$

Proof: Let $s$ be a solution of the system (7.5), (7.6). We define a pair $\{p, q\}$ by

$$
\left(\begin{array}{c}
p(z) \\
q(z)
\end{array}\right)=\Theta^{-1}(z)\left(\begin{array}{c}
s(z) \\
I_{\mathcal{G}}
\end{array}\right) .
$$

Then

$$
\left(\begin{array}{c}
s(z) \\
I_{\mathcal{G}}
\end{array}\right)=\Theta(z)\left(\begin{array}{c}
p(z) \\
q(z)
\end{array}\right)=\left(\begin{array}{c}
\Theta_{11}(z) p(z)+\Theta_{12}(z) q(z) \\
\Theta_{21}(z) p(z)+\Theta_{22}(z) q(z)
\end{array}\right)
$$

and in particular, $s$ admits a representation (7.10). On the other hand the latter equality implies $\Theta_{21}(z) p(z)+\Theta_{22}(z) q(z) \equiv I_{\mathcal{G}}$ and thus, the condition (7.11) is fulfilled. It remains to show that the pair $\{p, q\}$ defined by (7.12) is a Stieltjes pair. 
To do that we substitute (7.12) into (7.5), (7.6) and get the inequalities (7.2), (7.3) for $p$ and $q$. Next, it follows from (6.4) and (6.19) that

$$
\Theta^{-1}(z)=J N^{*} J\left\{I_{\mathcal{G} \oplus \mathcal{G}}+(z-\mu)\left(\begin{array}{c}
-\Pi_{1}^{*} C^{*} \\
\Pi_{2}^{*}
\end{array}\right) \Gamma(\mu) K^{-1} \Gamma(z)\left(\Pi_{2}, C \Pi_{1}\right)\right\} .
$$

By assumptions on $\Gamma$, the function $\Theta^{-1}(z)$ is boundedly invertible for all $z \in \mathbb{C} \backslash \mathcal{Z}$ and therefore, for every choice of $z \in \mathbb{C} \backslash \mathcal{Z}$ and $f \in \mathcal{G}$,

$$
\begin{aligned}
\left\langle\left(p(z)^{*} p(z)+q(z)^{*} q(z)\right) f, f\right\rangle_{\mathcal{G}} & =\left\langle\left(\begin{array}{c}
p(z) \\
q(z)
\end{array}\right) f,\left(\begin{array}{c}
p(z) \\
q(z)
\end{array}\right) f\right\rangle_{\mathcal{G} \oplus \mathcal{G}} \\
& \geq\left\langle\Theta^{-1}(z)\left(\begin{array}{c}
s(z) \\
I_{\mathcal{G}}
\end{array}\right) f, \Theta^{-1}(z)\left(\begin{array}{c}
s(z) \\
I_{\mathcal{G}}
\end{array}\right) f\right\rangle_{\mathcal{G} \oplus \mathcal{G}} \\
& \geq \varepsilon(z)\left\langle\left(\begin{array}{c}
s(z) \\
I_{\mathcal{G}}
\end{array}\right) f,\left(\begin{array}{c}
s(z) \\
I_{\mathcal{G}}
\end{array}\right) f\right\rangle_{\mathcal{G} \oplus \mathcal{G}} \geq \varepsilon(z)\langle f, f\rangle_{\mathcal{G}} .
\end{aligned}
$$

Therefore, $\left(p(z)^{*} p(z)+q(z)^{*} q(z)\right)$ is boundedly invertible for all $z \in \mathbb{C} \backslash \mathcal{Z}$ and by Definition 7.1, $\{p, q\}$ belongs to $\overline{\mathcal{S}}(\mathcal{G})$.

Conversely, let $\{p, q\}$ be any Stieltjes pair satisfying (7.11) and let $s$ be of the form (7.10). Rewriting (7.10) in the following equivalent form

$$
\left(\begin{array}{c}
p(z) \\
q(z)
\end{array}\right)=\Theta^{-1}(z)\left(\begin{array}{c}
s(z) \\
I_{\mathcal{G}}
\end{array}\right) v(z) \quad\left(v(z)=\Theta_{21}(z) p(z)+\Theta_{22}(z) q(z)\right)
$$

and substituting (7.13) into inequalities (7.2), (7.3) (which are in force since $\{p, q\} \in \overline{\mathcal{S}}(\mathcal{G})$ ) we obtain

$$
\begin{array}{r}
v(z)^{*}\left(s(z)^{*}, I_{\mathcal{G}}\right) \frac{\Theta(z)^{-*} J \Theta^{-1}(z)}{i(\bar{z}-z)}\left(\begin{array}{c}
s(z) \\
I_{\mathcal{G}}
\end{array}\right) v(z) \geq 0, \\
v(z)^{*}\left(s(z)^{*}, I_{\mathcal{G}}\right) \frac{\Theta(z)^{-*} P(z)^{*} J P(z) \Theta^{-1}(z)}{i(\bar{z}-z)}\left(\begin{array}{c}
s(z) \\
I_{\mathcal{G}}
\end{array}\right) v(z) \geq 0 .
\end{array}
$$

By (7.11), $v(z)$ is boundedly invertible at every point $z \in \mathbb{C} \backslash \mathbb{R}_{+}$and therefore, the two last inequalities are equivalent to (7.5) and (7.6), respectively. Thus, the function $s$ of the form (7.10) satisfies the inequalities (7.5) and (7.6).

Remark 7.4 It is easily seen that two Stieltjes pairs $\{p, q\}$ and $\left\{p_{1}, q_{1}\right\}$ lead by the transformation (7.10) to the same function $s(z)$ if and only if these pairs are equivalent (in the sense of (7.4)).

Note also that for the case $\operatorname{dim} \mathcal{G}<\infty$, the condition (7.11) is fulfilled automatically for every Stieltjes pair $\{p, q\}$ and at almost every point $z \in \mathbb{C} \backslash \mathbb{R}_{+}$(see [11]).

\section{Two-sided residue interpolation problem}

In the last four sections we apply the preceding analysis to several concrete interpolation problems. The first example is the two-sided residue interpolation problem suggested by A. 
Nudelman in [22] and considered for Stieltjes functions in [2].

The data set for an interpolation problem consists of three separable Hilbert spaces $\mathcal{H}_{\zeta}, \mathcal{H}_{\pi}$, $\mathcal{G}$ and of an ordered collection

$$
\Omega=\left\{C_{+}, C_{-}, A_{\pi}, A_{\zeta}, B_{+}, B_{-}, \Upsilon\right\}
$$

of seven operators

$$
C_{-}, C_{+} \in\left\{\mathcal{H}_{\pi} ; \mathcal{G}\right\}, A_{\pi} \in\left\{\mathcal{H}_{\pi} ; \mathcal{H}_{\pi}\right\}, A_{\zeta} \in\left\{\mathcal{H}_{\zeta} ; \mathcal{H}_{\zeta}\right\}, B_{+}, B_{-} \in\left\{\mathcal{G} ; \mathcal{H}_{\zeta}\right\}, \Upsilon \in\left\{\mathcal{H}_{\pi} ; \mathcal{H}_{\zeta}\right\}
$$

such that

$$
\operatorname{spec} A_{\zeta} \bigcup \operatorname{spec} A_{\pi} \subset \mathbb{C}_{+}, \quad \operatorname{Ran}\left(B_{+}^{*}, C_{-}\right)=\mathcal{G}
$$

and the following identity holds

$$
A_{\zeta} \Upsilon-\Upsilon A_{\pi}=B_{-} C_{-}-B_{+} C_{+} .
$$

Problem 8.1 Given a set $\Omega$ of operators (8.1) find all functions $s \in \mathcal{S}(\mathcal{G})$ such that

$$
\begin{aligned}
\oint_{\mathcal{L}}\left(z I-A_{\zeta}\right)^{-1} B_{+} s(z) d z & =B_{-} \\
\oint_{\mathcal{L}} s(z) C_{-}\left(z I-A_{\pi}\right)^{-1} d z & =C_{+} \\
\oint_{\mathcal{L}}\left(z I-A_{\zeta}\right)^{-1} B_{+} s(z) C_{-}\left(z I-A_{\pi}\right)^{-1} d z & =\Upsilon
\end{aligned}
$$

where $\mathcal{L} \in \mathbb{C}_{+}$is a closed contour around $\operatorname{spec} A_{\zeta} \cup \operatorname{spec} A_{\pi}$.

Such a contour exists since $\operatorname{spec} A_{\zeta} \cup \operatorname{spec} A_{\pi}$ is a closed bounded set separated from the real axis:

$$
\operatorname{dist}\left(\operatorname{spec} A_{\zeta} \cup \operatorname{spec} A_{\pi} ; \mathbb{R}\right)>0 \text {. }
$$

Condition (8.6) is added to the left-sided condition (8.4) and the right-sided condition (8.5) to take into account possible intersections of the spectra of the operators $A_{\zeta}$ and $A_{\pi}$.

Note that the equality (8.3) follows immediately from (8.4)-(8.6) and is therefore, a necessary condition for Problem 8.1 to be solvable. In the case when $\operatorname{spec} A_{\zeta} \cap \operatorname{spec} A_{\pi}=\{\emptyset\}$, the operator $\Upsilon$ is uniquely defined by (8.3) and therefore it need not to be preassigned as a part of interpolation data (8.1).

Note also that in the case when spectra of $A_{\zeta}$ and $A_{\pi}$ consist of finite number of isolated points, the contour integrals in (8.4)-(8.6) are equal to sums of residues of the corresponding functions taken all over $\mathbb{C}_{+}$. In such residue form Problem 8.1 appears in [2].

Substituting integral representation (2.2) of a Stieltjes function $s$ into (8.4)-(8.6) and using operator calculus we obtain the equivalent conditions

$$
\begin{aligned}
B_{+} \gamma+\int_{0}^{\infty}\left(\lambda I-A_{\zeta}\right)^{-1} B_{+} d \sigma(\lambda) & =B_{-} \\
\gamma C_{-}+\int_{0}^{\infty} d \sigma(\lambda) C_{-}\left(\lambda I-A_{\pi}\right)^{-1} & =C_{+} \\
\int_{0}^{\infty}\left(\lambda I-A_{\zeta}\right)^{-1} B_{+} d \sigma(\lambda) C_{-}\left(\lambda I-A_{\pi}\right)^{-1} & =\Upsilon
\end{aligned}
$$


which are expressed in terms of the measure $d \sigma$ associated with $s$. As the integral in (2.2), the integrals in (8.8)-(8.10) converge in the weak sense.

To reduce Problem 8.1 to Problem 1.2 we introduce several bounded operators which are uniquely defined by the interpolation data (8.1). These are:

$$
\begin{aligned}
K_{\zeta} & =\int_{0}^{\infty}\left(\lambda I-A_{\zeta}\right)^{-1} B_{+} d \sigma(\lambda) B_{+}^{*}\left(\lambda I-A_{\zeta}^{*}\right)^{-1} \\
K_{\pi} & =\int_{0}^{\infty}\left(\lambda I-A_{\pi}^{*}\right)^{-1} C_{-}^{*} d \sigma(\lambda) C_{-}\left(\lambda I-A_{\pi}\right)^{-1} \\
\widetilde{K}_{\zeta} & =\int_{0}^{\infty} \lambda\left(\lambda I-A_{\zeta}\right)^{-1} B_{+} d \sigma(\lambda) B_{+}^{*}\left(\lambda I-A_{\zeta}^{*}\right)^{-1}+B_{+} \gamma B_{+}^{*} \\
\widetilde{K}_{\pi} & =\int_{0}^{\infty} \lambda\left(\lambda I-A_{\pi}^{*}\right)^{-1} C_{-}^{*} d \sigma(\lambda) C_{-}\left(\lambda I-A_{\pi}\right)^{-1}+C_{-}^{*} \gamma C_{-} \\
\widetilde{\Upsilon} & =\int_{0}^{\infty} \lambda\left(\lambda I-A_{\zeta}\right)^{-1} B_{+} d \sigma(\lambda) C_{-}\left(\lambda I-A_{\pi}\right)^{-1}+B_{+} \gamma C_{-} .
\end{aligned}
$$

The weak convergence of all integrals in (8.11)-(8.15) follows from the convergence of integrals (8.8)-(8.10), in view of (8.7) and since

$$
\left\|\lambda\left(\lambda I_{\mathcal{H}_{\zeta}}-A_{\zeta}\right)^{-1}-I_{\mathcal{H}_{\zeta}}\right\| \rightarrow 0, \quad\left\|\lambda\left(\lambda I_{\mathcal{H}_{\pi}}-A_{\pi}\right)^{-1}-I_{\mathcal{H}_{\pi}}\right\| \rightarrow 0 \quad(\lambda \rightarrow \infty)
$$

Assumptions on spectra of operators $A_{\zeta}$ and $A_{\pi}$ ensure that the Lyapunov equations

$$
A_{\zeta} K_{\zeta}-K_{\zeta} A_{\zeta}^{*}=B_{-} B_{+}^{*}-B_{+} B_{-}^{*}, \quad A_{\pi}^{*} K_{\pi}-K_{\pi} A_{\pi}=C_{+}^{*} C_{-}-C_{-}^{*} C_{+}
$$

have the unique solutions $K_{\zeta}$ and $K_{\pi}$. It follows from (8.8), (8.9) that the operators $K_{\zeta}$ and $K_{\pi}$ defined by (8.11) and (8.12) satisfy these Lyapunov equations and are therefore uniquely defined by the interpolation data (8.1). The equalities

$$
\widetilde{K}_{\zeta}=A_{\zeta} K_{\zeta}+B_{+} B_{-}^{*}, \quad \widetilde{K}_{\pi}=A_{\pi}^{*} K_{\pi}+C_{-}^{*} C_{+}, \quad \widetilde{\Upsilon}=A_{\zeta} \Upsilon+B_{+} C_{+}
$$

follow from (8.8)-(8.15) and show in particular, that the operators $\widetilde{K}_{\zeta}, \widetilde{K}_{\pi}$ and $\widetilde{\Upsilon}$ also are uniquely defined by the interpolation data (8.1). Thus, equalities (8.11)-(8.15) can be considered as supplementary interpolation conditions for Problem 8.1 which are compatible with initial conditions (8.8)-(8.10). Another consequence of (8.16) is the identity

$$
\left(\begin{array}{cc}
A_{\zeta} & 0 \\
0 & A_{\pi}^{*}
\end{array}\right)\left(\begin{array}{cc}
K_{\zeta} & \Upsilon \\
\Upsilon^{*} & K_{\pi}
\end{array}\right)-\left(\begin{array}{cc}
\widetilde{K}_{\zeta} & \widetilde{\Upsilon} \\
\widetilde{\Upsilon}^{*} & \widetilde{K}_{\pi}
\end{array}\right)=-\left(\begin{array}{c}
B_{+} \\
C_{-}^{*}
\end{array}\right)\left(B_{-}^{*}, C_{+}\right)
$$

which means that the operators

$$
\begin{aligned}
K & =\left(\begin{array}{cc}
K_{\zeta} & \Upsilon \\
\Upsilon^{*} & K_{\pi}
\end{array}\right), \quad \widetilde{K}=\left(\begin{array}{cc}
\widetilde{K}_{\zeta} & \widetilde{\Upsilon} \\
\widetilde{\Upsilon}^{*} & \widetilde{K}_{\pi}
\end{array}\right), \quad \Pi_{1}=-\left(\begin{array}{c}
B_{-} \\
C_{+}^{*}
\end{array}\right), \quad \Pi_{2}=\left(\begin{array}{c}
B_{+} \\
C_{-}^{*}
\end{array}\right) \\
A & =\left(\begin{array}{cc}
A_{\zeta} & 0 \\
0 & A_{\pi}^{*}
\end{array}\right), \quad B=C=\left(\begin{array}{cc}
I_{\mathcal{H}_{\zeta}} & 0 \\
0 & I_{\mathcal{H}_{\pi}}
\end{array}\right)
\end{aligned}
$$


satisfy the identity (1.13). Let us consider Problem 1.2 for a special choice (8.17) of its data. Since $B$ and $C$ are now identity operators, it follows from (1.11) that $P_{\infty}=\widetilde{P}_{\infty}=0$ and $\widetilde{F}=\Pi_{2} \gamma^{\frac{1}{2}}$. Thus conditions (1.15)-(1.17) take the form

$$
\begin{aligned}
K & =\int_{0}^{\infty} \Gamma(\lambda) \Pi_{2} d \sigma(\lambda) \Pi_{2}^{*} \Gamma(\lambda)^{*}, \quad \widetilde{K}=\int_{0}^{\infty} \lambda \Gamma(\lambda) \Pi_{2} d \sigma(\lambda) \Pi_{2}^{*} \Gamma(\lambda)^{*}+\Pi_{2} \gamma \Pi_{2}^{*} \\
\Pi_{1} & =\int_{0}^{\infty} \Gamma(\lambda) \Pi_{2} d \sigma(\lambda)-\Pi_{2} \gamma
\end{aligned}
$$

and are equivalent, in view of (8.17), to (8.8)-(8.15). By Theorem 1.3, Problem 8.1 is solvable if and only if operators $K$ and $\widetilde{K}$ defined in (8.17) are both nonnegative. If they are strictly positive, the parametrization of all solutions of Problem 8.1 is given by Theorem 7.3.

A special choice of data (8.1) leads to the two-sided analogue of the classical Nevanlinna-Pick problem.

Problem 8.2 Given different points $z_{j} \in \mathbb{C}_{+}(j=1, \ldots, n)$, given spaces $\mathcal{G}_{j} \subseteq \mathcal{G}$ and given operators

$$
u_{j}, v_{j} \in\left\{\mathcal{G} ; \mathcal{G}_{j}\right\} ; \quad f_{j}, g_{j} \in\left\{\mathcal{G}_{j} ; \mathcal{G}\right\} ; \quad w_{j} \in\left\{\mathcal{G}_{j} ; \mathcal{G}_{j}\right\}
$$

such that

$$
u_{j} g_{j}=v_{j} f_{j},
$$

find all functions $s \in \mathcal{S}(\mathcal{G})$ satisfying the following interpolation conditions

$$
u_{j} s\left(z_{j}\right)=v_{j}, \quad s\left(z_{j}\right) f_{j}=g_{j}, \quad u_{j} s^{\prime}(z) f_{j}=w_{j} \quad(j=1, \ldots, n) .
$$

Let us define the spaces $\mathcal{H}_{\zeta}$ and $\mathcal{H}_{\pi}$ by

$$
\mathcal{H}_{\zeta}=\mathcal{H}_{\pi}=\bigoplus_{j=1}^{n} \mathcal{G}_{j}
$$

It is easily seen that conditions (8.19) are equivalent to residue conditions (8.4)-(8.6) for

$$
\begin{aligned}
& C_{-}=\left(f_{1}, \ldots, f_{n}\right), \quad C_{+}=\left(g_{1}, \ldots, g_{n}\right), \quad A_{\zeta}=A_{\pi}=\left(\begin{array}{cc}
z_{1} I_{\mathcal{G}_{1}} & \\
& \\
& z_{n} I_{\mathcal{G}_{n}}
\end{array}\right) \\
& B_{+}=\left(\begin{array}{c}
u_{1} \\
\vdots \\
u_{n}
\end{array}\right), \quad B_{-}=\left(\begin{array}{c}
v_{1} \\
\vdots \\
v_{n}
\end{array}\right), \quad \Upsilon=\left(\Upsilon_{j k}\right)_{j, k=1}^{n}, \quad \Upsilon_{j k}=\left\{\begin{array}{cc}
w_{j}, & j=k \\
\frac{u_{j} g_{k}-v_{j} f_{k}}{z_{j}-z_{k}}, & j \neq k
\end{array}\right.
\end{aligned}
$$

where all the operators are given in the block matrix form with respect to direct sum (8.20). Note that the identity (8.3) for the mentioned operators is provided by conditions (8.18). 


\subsection{Interpolation at negative points}

Since Stieltjes functions are analytic on $\mathbb{R}_{-}$, interpolation conditions at negative points can be put into consideration as well as nonreal ones. However, the nonnegativity of Stieltjes functions on $\mathbb{R}_{-}$induces some pecularities of such a problem.

Problem 8.3 Let $A, K \in\{\mathcal{H} ; \mathcal{H}\}$ and $B_{+}, B_{-} \in\{\mathcal{G} ; \mathcal{H}\}$ be operators such that

$$
\operatorname{spec} A \subset \mathbb{R}_{-}, \quad \operatorname{Ran} B_{+}^{*}=\mathcal{G} \quad \text { and } \quad A K-K A^{*}=B_{-} B_{+}^{*}-B_{+} B_{-}^{*} .
$$

Find all functions $s \in \mathcal{S}(\mathcal{G})$ satisfying the interpolation conditions

$$
\begin{aligned}
\oint_{\mathcal{L}}(z I-A)^{-1} B_{+} s(z) d z & =B_{-} \\
\oint_{\mathcal{L}}(z I-A)^{-1} B_{+} s(z) B_{+}^{*}(z I-A)^{-1} d z & =K
\end{aligned}
$$

where $\mathcal{L} \in \mathbb{C} \backslash \mathbb{R}_{+}$is a closed contour around $\operatorname{spec} A$.

Since $s(z)$ is selfadjoint for $z<0$, the left-sided condition (8.21) is equivalent to the rightsided condition (8.5) for $A_{\pi}=A, C_{-}=B_{+}^{*}$ and $C_{+}=B_{-}^{*}$. Therefore, (8.22) is the analogue of the two-sided condition (8.6) for the present situation. Reproducing arguments from the previous subsection we conclude that conditions $(8.21),(8.22)$ are equivalent to conditions (1.15)-(1.17) for

$$
B=C=I_{\mathcal{H}}, \quad \Pi_{2}=B_{+}, \quad \Pi_{1}=B_{-}, \quad \widetilde{K}=A K+B_{+} B_{-}^{*}
$$

and therefore Problem 8.3 is a particular case of Problem 1.2.

\section{Boundary interpolation problem}

In general, a Stieltjes function $s$ is not analytic on $\mathbb{R}_{+}$. However, as a function of bounded type, it has weak boundary limits

$$
s(x)=\lim _{\varepsilon \rightarrow+0} s(x+i \varepsilon)=\lim _{\varepsilon \rightarrow+0} s(x-i \varepsilon)^{*}
$$

at almost all (with respect to the Lebesgue measure $d \lambda$ ) points $x \in \mathbb{R}_{+}$. The boundary interpolation problem involves finding all Stieltjes functions which take prescribed boundary values at prescribed points on $\mathbb{R}_{+}$. In order to include the boundary interpolation problem into the general scheme we require more: the existence of the weak limit

$$
\lim _{\varepsilon \rightarrow 0} \frac{s\left(\lambda_{j}+i \varepsilon\right)-s\left(\lambda_{j}+i \varepsilon\right)^{*}}{2 i \varepsilon} \in\{\mathcal{G} ; \mathcal{G}\}
$$

at every point $\lambda_{j}$ of interpolation. Under such an assumption, boundary values $s\left(\lambda_{j}\right)$ exist and are selfadjoint. 
Problem 9.1 Given points $\lambda_{j} \in \mathbb{R}_{+}(j=1, \ldots, n)$, given selfadjoint operators $s_{j} \in\{\mathcal{G} ; \mathcal{G}\}$ and nonnegative operators $w_{j} \in\{\mathcal{G} ; \mathcal{G}\}$ find all functions $s \in \mathcal{S}(\mathcal{G})$ such that

$$
\lim _{\varepsilon \rightarrow 0} s\left(\lambda_{j}+i \varepsilon\right)=s_{j}, \quad \lim _{\varepsilon \rightarrow 0} \frac{s\left(\lambda_{j}+i \varepsilon\right)-s\left(\lambda_{j}+i \varepsilon\right)^{*}}{2 i \varepsilon} \leq w_{j} \quad(j=1, \ldots, n) .
$$

Let $s$ be a Stieltjes function satisfying (9.2) and let (2.2) be its integral representation with a $\{\mathcal{G} ; \mathcal{G}\}$-valued nonnegative measure $d \sigma$ satisfying (2.3). Then the assumption (9.1) is equivalent to

$$
\lim _{\varepsilon \rightarrow 0} \int_{0}^{\infty} \frac{d \sigma(\lambda)}{\left(\lambda-\lambda_{j}\right)^{2}+\varepsilon^{2}} \in\{\mathcal{G} ; \mathcal{G}\} \quad(j=1, \ldots, n),
$$

while conditions (9.2) are expressed in terms of the representing measure as

$$
\gamma+\lim _{\varepsilon \rightarrow 0} \int_{0}^{\infty} \frac{d \sigma(\lambda)}{\lambda-\lambda_{j}-i \varepsilon}=s_{j}, \quad \lim _{\varepsilon \rightarrow 0} \int_{0}^{\infty} \frac{d \sigma(\lambda)}{\left(\lambda-\lambda_{j}\right)^{2}+\varepsilon^{2}} \leq w_{j} \quad(j=1, \ldots, n) .
$$

Since the limits in (9.3) represent bounded operators,

$$
\lim _{\varepsilon \rightarrow 0} \int_{0}^{\infty} \frac{\varepsilon d \sigma(\lambda)}{\left(\lambda-\lambda_{j}\right)^{2}+\varepsilon^{2}}=0 .
$$

On the other hand, the weak convergence of the integral $\int_{0}^{\infty} \frac{d \sigma(\lambda)}{\left(\lambda-\lambda_{j}\right)^{2}}$ follows from (9.3) by Fatou's lemma. Applying Lebesgue's dominated convergence theorem we obtain that

$$
\lim _{\varepsilon \rightarrow 0} \int_{0}^{\infty} \frac{d \sigma(\lambda)}{\left(\lambda-\lambda_{j}\right)^{2}+\varepsilon^{2}}=\int_{0}^{\infty} \frac{d \sigma(\lambda)}{\left(\lambda-\lambda_{j}\right)^{2}} .
$$

Using the inequality $\frac{\lambda}{\left(\lambda-\lambda_{j}\right)^{2}}<\frac{2}{\lambda+1}$ which holds for all $\lambda>4 \lambda_{j}+1$ we get

$$
\begin{aligned}
\int_{0}^{\infty} \frac{\lambda d \sigma(\lambda)}{\left(\lambda-\lambda_{j}\right)^{2}} & \leq \int_{0}^{4 \lambda_{j}+1} \frac{4 \lambda_{j}+1}{\left(\lambda-\lambda_{j}\right)^{2}} d \sigma(\lambda)+\int_{4 \lambda_{j}+1}^{\infty} \frac{2 d \sigma(\lambda)}{\lambda+1} \\
& \leq\left(4 \lambda_{j}+1\right) \int_{0}^{\infty} \frac{d \sigma(\lambda)}{\left(\lambda-\lambda_{j}\right)^{2}}+2 \int_{0}^{\infty} \frac{d \sigma(\lambda)}{\lambda+1} .
\end{aligned}
$$

Since $d \sigma$ satisfies (2.3), the last estimate implies the weak convergence of the integral $\int_{0}^{\infty} \frac{\lambda d \sigma(\lambda)}{\left(\lambda-\lambda_{j}\right)^{2}}$ and again by Lebesgue's dominated convergence theorem we conclude that

$$
\lim _{\varepsilon \rightarrow 0} \int_{0}^{\infty} \frac{\lambda d \sigma(\lambda)}{\left(\lambda-\lambda_{j}\right)^{2}+\varepsilon^{2}}=\int_{0}^{\infty} \frac{\lambda d \sigma(\lambda)}{\left(\lambda-\lambda_{j}\right)^{2}} .
$$

Now it follows from (9.5)-(9.7) that

$$
\begin{aligned}
\lim _{\varepsilon \rightarrow 0} \int_{0}^{\infty} \frac{d \sigma(\lambda)}{\lambda-\lambda_{j}-i \varepsilon} & =\lim _{\varepsilon \rightarrow 0} \int_{0}^{\infty} \frac{\lambda d \sigma(\lambda)}{\left(\lambda-\lambda_{j}\right)^{2}+\varepsilon^{2}}-\lim _{\varepsilon \rightarrow 0} \int_{0}^{\infty} \frac{\lambda_{j}-i \varepsilon}{\left(\lambda-\lambda_{j}\right)^{2}+\varepsilon^{2}} d \sigma(\lambda) \\
& =\int_{0}^{\infty} \frac{\lambda d \sigma(\lambda)}{\left(\lambda-\lambda_{j}\right)^{2}}-\int_{0}^{\infty} \frac{\lambda_{j} d \sigma(\lambda)}{\left(\lambda-\lambda_{j}\right)^{2}}=\int_{0}^{\infty} \frac{d \sigma(\lambda)}{\lambda-\lambda_{j}} .
\end{aligned}
$$


Taking advantage of (9.6) and (9.8) we rewrite conditions (9.4) in the form

$$
\gamma+\int_{0}^{\infty} \frac{d \sigma(\lambda)}{\lambda-\lambda_{j}}=s_{j}, \quad \int_{0}^{\infty} \frac{d \sigma(\lambda)}{\left(\lambda-\lambda_{j}\right)^{2}} \leq w_{j} \quad(j=1, \ldots, n) .
$$

The supplementary conditions

$$
\begin{gathered}
\int_{0}^{\infty} \frac{d \sigma(\lambda)}{\left(\lambda-\lambda_{j}\right)\left(\lambda-\lambda_{\ell}\right)}=\frac{s_{j}-s_{\ell}}{\lambda_{j}-\lambda_{\ell}}, \quad \gamma+\int_{0}^{\infty} \frac{\lambda d \sigma(\lambda)}{\left(\lambda-\lambda_{j}\right)^{2}} \leq \lambda_{j} w_{j}+s_{j} \\
\gamma+\int_{0}^{\infty} \frac{\lambda d \sigma(\lambda)}{\left(\lambda-\lambda_{j}\right)\left(\lambda-\lambda_{\ell}\right)}=\frac{\lambda_{j} s_{j}-\lambda_{\ell} s_{\ell}}{\lambda_{j}-\lambda_{\ell}}, \quad(j, \ell=1, \ldots, n ; j \neq \ell)
\end{gathered}
$$

as well as convergence of the corresponding integrals, follow immediately from (9.9). Let us consider Problem 1.1 for

$$
\mathcal{H}=\widetilde{\mathcal{H}}=\underbrace{\mathcal{G} \oplus \cdots \oplus \mathcal{G}}_{n \text { times }}
$$

and for operators

$$
\begin{array}{rlr}
A=\left(\begin{array}{rrr}
\lambda_{1} I_{\mathcal{G}} & & \\
& \ddots & \\
& & \lambda_{n} I_{\mathcal{G}}
\end{array}\right), \quad B=C=I_{\mathcal{H}}, \quad \Pi_{1}=-\left(\begin{array}{c}
s_{1} \\
\vdots \\
s_{n}
\end{array}\right), \quad \Pi_{2}=\left(\begin{array}{c}
I_{\mathcal{G}} \\
\vdots \\
I_{\mathcal{G}}
\end{array}\right) \\
K=\left(K_{j \ell}\right)_{j, \ell=1}^{n} \quad \text { with } \quad K_{j \ell}=\left\{\begin{array}{cc}
w_{j}, & j=\ell \\
\frac{s_{j}-s_{\ell}}{\lambda_{j}-\lambda_{\ell}}, & j \neq \ell
\end{array}\right. \\
\widetilde{K}=\left(\widetilde{K}_{j \ell}\right)_{j, \ell=1}^{n} & \text { with } \quad \widetilde{K}_{j \ell}= \begin{cases}\lambda_{j} w_{j}+s_{j}, & j=\ell \\
\frac{\lambda_{j} s_{j}-\lambda_{\ell} s_{\ell}}{\lambda_{j}-\lambda_{\ell}}, & j \neq \ell\end{cases}
\end{array}
$$

which are presented in the block forms with respect to decompositions (9.11) of $\mathcal{H}$ and $\widetilde{\mathcal{H}}$. The identity (1.13) for these operators can be easily verified and according to (1.2),

$$
\Gamma(\lambda) \Pi_{2}=\left(A-\lambda I_{\mathcal{H}}\right)^{-1} \Pi_{2}=\left(\begin{array}{c}
\left(\lambda_{1}-\lambda\right)^{-1} \\
\vdots \\
\left(\lambda_{n}-\lambda\right)^{-1}
\end{array}\right) .
$$

Conditions (1.11) and (1.12) imply

$$
P_{\infty}=\widetilde{P}_{\infty}=0, \quad \widetilde{F}=\Pi_{2} \gamma^{\frac{1}{2}} x_{i}=0
$$

and provide the operator $P_{i}+F_{i} F_{i}^{*}$ to be of the form

$$
P_{i}+F_{i} F_{i}^{*}=\left(\begin{array}{ccccc}
0 & \cdots & 0 & \cdots & 0 \\
\vdots & \ddots & & & \vdots \\
0 & \cdots & p_{i} & \cdots & 0 \\
\vdots & & & \ddots & \vdots \\
0 & \cdots & 0 & \cdots & 0
\end{array}\right) \quad\left(0 \leq p_{i} \in\{\mathcal{G} ; \mathcal{G}\}\right)
$$


(i.e., with the only possibly nonzero $i i$-entry $p_{i}$ in the block representation with respect to decomposition (9.11)). Substituting (9.12)-(9.15) into (1.8)-(1.12) and comparing corresponding $\{\mathcal{G} ; \mathcal{G}\}$-blocks we get

$$
\begin{aligned}
& K_{j \ell}=\left\{\begin{array}{cc}
\int_{0}^{\infty} \frac{d \sigma(\lambda)}{\left(\lambda-\lambda_{j}\right)^{2}}+p_{j} & j=\ell \\
\int_{0}^{\infty} \frac{d \sigma(\lambda)}{\left(\lambda-\lambda_{j}\right)\left(\lambda-\lambda_{\ell}\right)} & j \neq \ell
\end{array} \quad \widetilde{K}_{j \ell}=\left\{\begin{array}{cc}
\int_{0}^{\infty} \frac{\lambda d \sigma(\lambda)}{\left(\lambda-\lambda_{j}\right)^{2}}+\gamma+\lambda_{j} p_{j} & j=\ell \\
\int_{0}^{\infty} \frac{\lambda d \sigma(\lambda)}{\left(\lambda-\lambda_{j}\right)\left(\lambda-\lambda_{\ell}\right)}+\gamma & j \neq \ell
\end{array}\right.\right. \\
& \left(\Pi_{1}\right)_{j}=-s_{j}=\int_{0}^{\infty} \frac{d \sigma(\lambda)}{\lambda_{j}-\lambda}-\gamma .
\end{aligned}
$$

Taking into acccount the explicit formulas for $K_{j \ell}$ and $\widetilde{K}_{j \ell}$ it is easily seen that the obtained equalities are equivalent to (9.9), (9.10). Therefore Problem 1.1 with a special choice (9.12) of the data is equivalent to Problem 9.1. By Theorem 1.3, Problem 9.1 has a solution if and only if operators $K$ and $\widetilde{K}$ defined in (9.12) are nonnegative. If these operators are strictly positive, the set of all solutions of Problem 9.1 is described as in Theorem 7.3.

\section{Stieltjes moment problem}

The next example is the truncated Stieltjes moment problem which consists of the following.

Problem 10.1 Given nonnegative bounded operators $s_{0}, \ldots, s_{N}$ acting in a separable Hilbert space $\mathcal{G}$ find all nondecreasing $\{\mathcal{G} ; \mathcal{G}\}$-valued functions $\sigma(\lambda)$ on $\mathbb{R}_{+}$such that

$$
\int_{0}^{\infty} \lambda^{k} d \sigma(\lambda)=s_{k}(k=0, \ldots, N-1) \quad \text { and } \quad \int_{0}^{\infty} \lambda^{N} d \sigma(\lambda) \leq s_{N}
$$

where the indicated integrals converge in the weak sense.

There is a slight difference between the cases of even and odd $N$ which suggests to consider them separately. However, both of these cases are reduced to Problem 1.2 for a special choice of data (1.14).

1. Let $N=2 n+1$ and let

$$
\mathcal{H}=\widetilde{\mathcal{H}}=\underbrace{\mathcal{G} \oplus \cdots \oplus \mathcal{G}}_{(n+1) \text { times }} .
$$

We consider Problem 1.2 for $A=B=I_{\mathcal{H}}$ and

$$
\begin{aligned}
& K=\left(\begin{array}{cccc}
s_{0} & s_{1} & \cdots & s_{n} \\
s_{1} & s_{2} & \cdots & s_{n+1} \\
\vdots & \vdots & & \vdots \\
s_{n} & s_{n+1} & \cdots & s_{2 n}
\end{array}\right), \quad \widetilde{K}=\left(\begin{array}{cccc}
s_{1} & s_{2} & \cdots & s_{n+1} \\
s_{2} & s_{3} & \cdots & s_{n+2} \\
\vdots & \vdots & & \vdots \\
s_{n+1} & s_{n+2} & \cdots & s_{2 n+1}
\end{array}\right) \\
& \Pi_{1}=\left(\begin{array}{c}
s_{0} \\
\vdots \\
s_{n}
\end{array}\right), \quad \Pi_{2}=\left(\begin{array}{c}
I_{\mathcal{G}} \\
0 \\
\vdots \\
0
\end{array}\right), \quad C=\left(\begin{array}{cccc}
0 & 0 & \ldots & 0 \\
I_{\mathcal{G}} & 0 & \ddots & \vdots \\
\vdots & \ddots & \ddots & 0 \\
0 & \ldots & I_{\mathcal{G}} & 0
\end{array}\right)
\end{aligned}
$$


where all the operators are presented in the block-matrix form with respect to decomposition (10.2). It is easily verified that these operators satisfy (1.13) and, according to (1.2),

$$
\Gamma(z) \Pi_{2}=\left(\begin{array}{c}
I_{\mathcal{G}} \\
z I_{\mathcal{G}} \\
\vdots \\
z^{n} I_{\mathcal{G}}
\end{array}\right)
$$

The operators $P_{\infty}, \widetilde{P}_{\infty}, \widetilde{F}$ and $\gamma$ satisfying (1.11), necessarily are of the form

$$
\gamma=0, \quad P_{\infty}=0, \quad \widetilde{P}_{\infty}+\widetilde{F} \widetilde{F}^{*}=\left(\begin{array}{cccc}
0 & \cdots & 0 & 0 \\
\vdots & & \vdots & \vdots \\
0 & \cdots & 0 & 0 \\
0 & \cdots & 0 & s
\end{array}\right) \quad(0 \leq s \in\{\mathcal{G} ; \mathcal{G}\}) .
$$

Substituting (10.3)-(10.5) into (1.15)-(1.17) and comparing $\{\mathcal{G} ; \mathcal{G}\}$-blocks in the obtained operator equalities we get (10.1). Note that first $N-1$ conditions in (10.1) are equivalent to (1.15), whereas the inequality for the last moment follows from the integral representation of the right bottom $\{\mathcal{G} ; \mathcal{G}\}$-block of $\widetilde{K}$ in (1.16). All other conditions which can be deduced from (1.16) and (1.17) are superfluous but compatible with conditions (10.1).

Let now $N=2 n$, let

$$
\mathcal{H}=\underbrace{\mathcal{G} \oplus \cdots \oplus \mathcal{G}}_{(n+1) \text { times }}, \quad \widetilde{\mathcal{H}}=\underbrace{\mathcal{G} \oplus \cdots \oplus \mathcal{G}}_{n \text { times }}
$$

and let us consider Problem 1.2 for operators

$$
\begin{gathered}
K=\left(\begin{array}{cccc}
s_{0} & s_{1} & \cdots & s_{n} \\
s_{1} & s_{2} & \cdots & s_{n+1} \\
\vdots & \vdots & & \vdots \\
s_{n} & s_{n+1} & \cdots & s_{2 n}
\end{array}\right), \quad \widetilde{K}=\left(\begin{array}{cccc}
s_{1} & s_{2} & \cdots & s_{n} \\
s_{2} & s_{3} & \cdots & s_{n+1} \\
\vdots & \vdots & & \vdots \\
s_{n} & s_{n+1} & \cdots & s_{2 n-1}
\end{array}\right), \quad \Pi_{1}=\left(\begin{array}{c}
s_{0} \\
s_{1} \\
\vdots \\
s_{n-1}
\end{array}\right) \\
\Pi_{2}=\left(\begin{array}{c}
I_{\mathcal{G}} \\
\vdots \\
0
\end{array}\right), \quad A=I_{\mathcal{H}}, \quad C=\left(\begin{array}{cccc}
0 & & \cdots & 0 \\
I_{\mathcal{G}} & 0 & & \\
0 & I_{\mathcal{G}} & \ddots & \vdots \\
\vdots & \ddots & \ddots & 0 \\
0 & \ldots & 0 & I_{\mathcal{G}}
\end{array}\right), \quad B=\left(\begin{array}{ccccc}
I_{\mathcal{G}} & 0 & \ldots & 0 \\
0 & I_{\mathcal{G}} & \ddots & \vdots \\
\vdots & & \ddots & 0 \\
0 & \ldots & 0 & I_{\mathcal{G}} & 0
\end{array}\right)
\end{gathered}
$$

which satisfy the identity (1.13). By (1.2), $\Gamma(z) \Pi_{2}$ is as in (10.4). Conditions (1.11) provide $\widetilde{P}_{\infty}, \widetilde{F}$ and $\gamma$ to be zero operators while $P_{\infty}$ should be of the form

$$
P_{\infty}=\left(\begin{array}{cccc}
0 & \cdots & 0 & 0 \\
\vdots & & \vdots & \vdots \\
0 & \cdots & 0 & 0 \\
0 & \cdots & 0 & s
\end{array}\right) \quad(0 \leq s \in\{\mathcal{G} ; \mathcal{G}\})
$$

Substituting (10.4), (10.6) and (10.7) into (1.15) we get (10.1). Besides, conditions (1.16), (1.17) are compatible with (10.1).

A number of more complicated examples of the moment problem type may be found in [5]. 


\section{Analogues of Krein's extension problem}

In this section we consider continuous interpolation problems on integral representations of functions generating positive integral operators in the Hilbert space $\mathrm{L}_{2}^{n}[0 ; \ell]$ of all $\mathbb{C}^{n}$-valued finctions with summable square

$$
\|f\|_{\mathrm{L}_{2}^{n}[0 ; \ell]}^{2}=\int_{0}^{\ell} f(\lambda)^{*} f(\lambda) d \lambda
$$

Example 11.1 Denote by $\mathcal{P}_{\ell}$ the set of all $\mathbb{C}^{n \times n}$-valued functions $k(x)$ continuous on $[-\ell ; \ell]$ and such that the bounded operators $K$ and $\widetilde{K}$ acting in $\mathrm{L}_{2}^{n}[0 ; \ell]$ by the rules

$$
(K f)(x)=\int_{0}^{\ell} k(x-t) f(t) d t, \quad(\widetilde{K} f)(x)=i \frac{d}{d x} \int_{0}^{\ell} k(x-t) f(t) d t,
$$

are nonnegative.

The condition $K \geq 0$ means that the function $k(x)$ is Hermitian positive on $[-\ell ; \ell]$ and therefore (see [20]), it admits a representation

$$
k(x)=\int_{-\infty}^{\infty} e^{-i x \lambda} d \sigma(\lambda)
$$

for a nonnegative $\mathbb{C}^{n \times n}$-valued measure $d \sigma(\lambda)$ satisfying condition (2.7). In particular, $k(x)=k(-x)^{*}$. We show that the additional condition $\widetilde{K} \geq 0$ provides the existence of a representing measure $d \sigma(\lambda)$ in (11.2) with the support in $\mathbb{R}_{+}$.

Let $C \in\left\{\mathrm{L}_{2}^{n}[0 ; \ell] ; \mathrm{L}_{2}^{n}[0 ; \ell]\right\}$ and $\Pi_{1}, \Pi_{2} \in\left\{\mathbb{C}^{n} ; \mathrm{L}_{2}^{n}[0 ; \ell]\right\}$ be the operators defined by

$$
(C f)(x)=-i \int_{0}^{x} f(t) d t, \quad\left(\Pi_{1} g\right)(x)=k(x) g, \quad\left(\Pi_{2} g\right)(x)=g
$$

for $f \in \mathrm{L}_{2}^{n}[0 ; \ell]$ and $g \in \mathbb{C}^{n}$. Then

$$
\begin{aligned}
\Pi_{1}^{*} f & =\int_{0}^{\ell} k(t)^{*} f(t) d t=\int_{0}^{\ell} k(-t) f(t) d t \\
(C \widetilde{K} f)(x) & =\int_{0}^{x} \frac{d}{d t} \int_{0}^{\ell} k(t-\xi) f(\xi) d \xi=\left.\int_{0}^{\ell} k(t-\xi) f(\xi) d \xi\right|_{0} ^{x} \\
& =\int_{0}^{\ell} k(x-\xi) f(\xi) d \xi-\int_{0}^{\ell} k(-\xi) f(\xi) d \xi
\end{aligned}
$$

and now it is easily seen that the operators from (11.1) and (11.3) satisfy the identity

$$
K-C \widetilde{K}=\Pi_{2} \Pi_{1}^{*}
$$

which is a particular case of the identity (1.13) corresponding to a choice $A=B=I_{\mathrm{L}_{2}^{n}[0 ; \ell]}$. According to (1.2),

$$
\left(\Gamma(\lambda) \Pi_{2} g\right)(x)=(I-\lambda C)^{-1} g=\sum_{j=0}^{\infty} \lambda^{j} C^{j} g=e^{-i \lambda x} g \quad\left(\forall g \in \mathbb{C}^{n}\right) .
$$


By Theorem 1.3, there exists a measure $d \sigma(\lambda)$ giving representations (1.15)-(1.17). Since operators $B$ and $C$ are injective, relations (1.11) provide $P_{\infty}$ and $\widetilde{P}_{\infty}$ to be zero operators. Next, let $\widetilde{F} \in\left\{\mathbb{C}^{n} ; \mathrm{L}_{2}^{n}[0 ; \ell]\right\}$ and $\gamma \in \mathbb{C}^{n \times n}$ be subject to

$$
C \widetilde{F} g=\Pi_{2} \gamma^{\frac{1}{2}} g \quad\left(\forall g \in \mathbb{C}^{n}\right) .
$$

Taking advantage of (11.3) we rewrite the latter equality as

$$
-i \int_{0}^{x}(\widetilde{F} g)(t) d t \equiv \gamma^{\frac{1}{2}} g=\text { const. }
$$

Therefore, $\widetilde{F} g$ is a zero element of $\mathrm{L}_{2}^{n}[0 ; \ell]$ and since $g$ is arbitrary, $\widetilde{F}=0$. This in turn, implies $\gamma=0$. On account of (11.3), (1.17) and (11.5),

$$
\left(\Pi_{1} g\right)(x)=k(x) g=\int_{0}^{\infty} \Gamma(\lambda) \Pi_{2} d \sigma(\lambda) g=\int_{0}^{\infty} e^{-i \lambda x} d \sigma(\lambda) g
$$

and since $g$ is an arbitrary vector from $\mathbb{C}^{n}$,

$$
k(x)=\int_{0}^{\infty} e^{-i x \lambda} d \sigma(\lambda) .
$$

As a solution of Problem 1.2, the measure $d \sigma$ should provide the convergence of the integral in the right side of (11.6). For $x=0$ this implies (2.7). Conversely, for every function $k$ of the form (11.6) the operators $K$ and $\widetilde{K}$ defined via (11.1) are nonnegative:

$$
\begin{aligned}
(K f, f)_{\mathrm{L}_{2}^{n}[0 ; \ell]} & =\int_{0}^{\ell} \int_{0}^{\ell} f(t)^{*} \int_{0}^{\infty} e^{i t \lambda} d \sigma(\lambda) e^{-i \tau \lambda} f(\tau) d t d \tau \geq 0 \\
(\widetilde{K} f, f)_{\mathrm{L}_{2}^{n}[0 ; \ell]} & =\int_{0}^{\ell} \int_{0}^{\ell} f(t)^{*} \int_{0}^{\infty} \lambda e^{i t \lambda} d \sigma(\lambda) e^{-i \tau \lambda} f(\tau) d t d \tau \geq 0
\end{aligned}
$$

and therefore, $k \in \mathcal{P}_{\ell}$. Moreover, the integral in (11.6) makes sense for all $x \in \mathbb{R}$ and therefore, $k$ can be extended to the whole real axis such that the corresponding integral operators $K$ and $\widetilde{K}$ will be still positive semidefinite. In fact the following theorem is proved.

Theorem 11.2 A $\mathbb{C}^{n \times n}$-valued function $k$ belongs to the class $\mathcal{P}_{\ell}$ if and only if it admits a representation (11.6) with a nonnegative $\mathbb{C}^{n \times n}$-valued measure d $\sigma$ satisfying condition (2.7). Every $k \in \mathcal{P}_{\ell}$ admits an extension to a function $\tilde{k} \in \mathcal{P}_{\infty}$.

Example 11.3 Denote by $\mathcal{K}_{\ell}$ the set of all $\mathbb{C}^{n \times n}$-valued functions $k(x)$ continuous on $[0 ; 2 \ell]$ and such that the bounded operators $K$ and $\widetilde{K}$ acting in $\mathrm{L}_{2}^{n}[0 ; \ell]$ by the rules

$$
\begin{aligned}
& (K f)(x)=\int_{0}^{\ell}(k(x+t)+k(|x-t|)) f(t) d t \\
& (\widetilde{K} f)(x)=-\frac{d^{2}}{d x^{2}} \int_{0}^{\ell}(k(x+t)+k(|x-t|)) f(t) d t
\end{aligned}
$$

are nonnegative. 
In particular, $k(x)=k(x)^{*}$. Let us define the operators $C, \Pi_{1}$ and $\Pi_{2}$ by

$$
(C f)(x)=-\int_{0}^{x}(x-t) f(t) d t, \quad\left(\Pi_{1} g\right)(x)=2 k(x) g, \quad\left(\Pi_{2} g\right)(x)=g
$$

for $f \in \mathrm{L}_{2}^{n}[0 ; \ell]$ and $g \in \mathbb{C}^{n}$. Since

$$
\Pi_{2} \Pi_{1}^{*} f=2 \Pi_{2} \int_{0}^{\ell} k(t)^{*} f(t) d t=2 \int_{0}^{\ell} k(t) f(t) d t \quad\left(f \in \mathrm{L}_{2}^{n}[0 ; \ell]\right)
$$

and

$$
\begin{aligned}
(C \widetilde{K} f)(x)= & \int_{0}^{x}(x-t) \frac{d^{2}}{d t^{2}} \int_{0}^{\ell}(k(t+\xi)+k(|t-\xi|)) f(\xi) d \xi \\
= & \left.(x-t) \frac{d}{d t} \int_{0}^{\ell}(k(t+\xi)+k(|t-\xi|)) f(\xi) d \xi\right|_{0} ^{x} \\
& +\left.\int_{0}^{\ell}(k(t+\xi)+k(|t-\xi|)) f(\xi) d \xi\right|_{0} ^{x} \\
= & \int_{0}^{\ell}(k(x+t)+k(|x-t|)) f(t) d t-2 \int_{0}^{\ell} k(t) f(t) d t
\end{aligned}
$$

the identity (11.4) holds for the operators introduced in (11.7) and (11.8). Applying the equality

$$
\left(C^{m} f\right)(x)=\frac{(-1)^{m}}{(2 m-1) !} \int_{0}^{x}(x-t)^{2 m-1} f(t) d t
$$

(which follows immediately from the definition (11.8) of the operator $C$ ) to the function $f(t)=g\left(g \in \mathbb{C}^{n}\right)$ we get

$$
\left(C^{m} g\right)(x)=\frac{(-1)^{m}}{(2 m-1) !} \int_{0}^{x}(x-t)^{2 m-1} d t g=\frac{(-1)^{m}}{2 m !} x^{2 m} g \quad(m \geq 1)
$$

and therefore,

$$
\left(\Gamma(\lambda) \Pi_{2} g\right)(x)=(I-\lambda C)^{-1} g=\sum_{m=0}^{\infty} \lambda^{m} C^{m} g=\sum_{m=0}^{\infty} \frac{(-1)^{m}}{2 m !} x^{2 m} \lambda^{m} g=\cos x \sqrt{\lambda} g .
$$

By Theorem 1.3, there exists a measure $d \sigma(\lambda)$ giving representations (1.15)-(1.17). As in Example 11.1, $P_{\infty}, \widetilde{P}_{\infty}$ and $\widetilde{F}$ satisfying (1.11), necessarily are zero operators. From (1.17) we get the integral representation of a function $k \in \mathcal{K}_{\ell}$ : by (11.8) and (11.11), it holds for every $g \in \mathbb{C}^{n}$ that

$$
\left(\Pi_{1} g\right)(x)=2 k(x) g=\int_{0}^{\infty} \Gamma(\lambda) \Pi_{2} d \sigma(\lambda) g=\int_{0}^{\infty} \cos x \sqrt{\lambda} d \sigma(\lambda) g
$$

and therefore,

$$
k(x)=\frac{1}{2} \int_{0}^{\infty} \cos x \sqrt{\lambda} d \sigma(\lambda)
$$


The representing measure $d \sigma$ provides the convergence of the integral in the right side of (11.12) and therefore $d \sigma$ satisfies condition (2.7). Conversely, for every function $k$ of the form (11.12) the operators $K$ and $\widetilde{K}$ defined via (11.7) are nonnegative:

$$
\begin{aligned}
& (K f, f)_{\mathrm{L}_{2}^{n}[0 ; \ell]}=\int_{0}^{\ell} \int_{0}^{\ell} f(t)^{*} \int_{0}^{\infty} \cos t \sqrt{\lambda} d \sigma(\lambda) \cos \tau \sqrt{\lambda} f(\tau) d t d \tau \geq 0 \\
& (\widetilde{K} f, f)_{\mathrm{L}_{2}^{n}[0 ; \ell]}=\int_{0}^{\ell} \int_{0}^{\ell} f(t)^{*} \int_{0}^{\infty} \lambda \cos t \sqrt{\lambda} d \sigma(\lambda) \cos \tau \sqrt{\lambda} f(\tau) d t d \tau \geq 0 .
\end{aligned}
$$

Note that the only positivity of the operator $K$ from (11.7) guarantees the existence of a representation

$$
k(x)=\frac{1}{2} \int_{-\infty}^{\infty} \cos x \sqrt{\lambda} d \sigma(\lambda)
$$

of the function $k$ for a nonnegative measure $d \sigma$ on the whole real axis (see [20]). To ensure the convergence of the integral, the representing measure has to satisfy besides (2.7) an appropriate condition on $\mathbb{R}_{-}$.

Example 11.4 Denote by $\mathcal{F}_{\ell}$ the set of all $\mathbb{C}^{n \times n}$-valued functions $k(x)$ piecewise continuous on $[-2 \ell ; 2 \ell]$, satisfying $k(x)=k(x)^{*}=-k(-x)$ and such that the bounded operators $K$ and $\widetilde{K}$ acting in $\mathrm{L}_{2}^{n}[0 ; \ell]$ by the rules

$$
\begin{aligned}
& (K f)(x)=\frac{d}{d x} \int_{0}^{\ell}(k(x-t)+k(x+t)) f(t) d t \\
& (\widetilde{K} f)(x)=\frac{d}{d x} \int_{0}^{\ell}(k(x-t)-k(x+t)) f(t) d t
\end{aligned}
$$

are nonnegative.

Let us define the operators $B, C, \Pi_{1}$ and $\Pi_{2}$ by

$$
(B f)(x)=\int_{0}^{x} f(t) d t, \quad(C f)(x)=-\int_{0}^{x} f(t) d t, \quad\left(\Pi_{1} g\right)(x)=2 k(x) g, \quad\left(\Pi_{2} g\right)(x)=g
$$

for $f \in \mathrm{L}_{2}^{n}[0 ; \ell]$ and $g \in \mathbb{C}^{n}$. The straightforward calculations show that

$$
\begin{aligned}
\left(K B^{*} f\right)(x) & =\int_{0}^{\ell}(k(x-t)-k(x+t)) f(t) d t, \quad \Pi_{2} \Pi_{1}^{*} f=2 \int_{0}^{\ell} k(t) f(t) d t \\
(C \widetilde{K})(x) & =\int_{0}^{\ell}(k(x-t)-k(x+t)) f(t) d t-2 \int_{0}^{\ell} k(t) f(t) d t
\end{aligned}
$$

and therefore,

$$
K B^{*}-C \widetilde{K}=\Pi_{2} \Pi_{1}^{*}
$$

Furthermore,

$$
(C B f)(x)=-\int_{0}^{x}(x-t) f(t) d t
$$

and using the calculations from the the previous example, we get

$$
\left(\Gamma(\lambda) \Pi_{2} g\right)(x)=(I-\lambda C B)^{-1} x=\cos x \sqrt{\lambda} g, \quad B\left(\Gamma(\lambda) \Pi_{2} g\right)(x)=\frac{\sin x \sqrt{\lambda}}{\sqrt{\lambda}} .
$$


As above, the operators $P_{\infty}, \widetilde{P}_{\infty}$ and $\widetilde{F}$ satisfying $(1.11)$ are zero operators. By Theorem 1.3 there exists a measure $d \sigma(\lambda)$ giving representations (1.15)-(1.17). ¿From (1.17) we get the integral representation of a function $k \in \mathcal{F}_{\ell}$ :

$$
k(x) g=\frac{1}{2}\left(\Pi_{1} g\right)(x)=\int_{0}^{\infty} B \Gamma(\lambda) \Pi_{2} d \sigma(\lambda) g=\int_{0}^{\infty} \frac{\sin x \sqrt{\lambda}}{\sqrt{\lambda}} d \sigma(\lambda) g .
$$

Moreover, for every function $k$ of the form (11.16) the operators $K$ and $\widetilde{K}$ defined via (11.13) are nonnegative:

$$
\begin{aligned}
(K f, f)_{\mathrm{L}_{2}^{n}[0 ; \ell]} & =\int_{0}^{\ell} \int_{0}^{\ell} f(t)^{*} \int_{0}^{\infty} \cos t \sqrt{\lambda} d \sigma(\lambda) \cos \tau \sqrt{\lambda} f(\tau) d t d \tau \geq 0 \\
(\widetilde{K} f, f)_{\mathrm{L}_{2}^{n}[0 ; \ell]} & =\int_{0}^{\ell} \int_{0}^{\ell} f(t)^{*} \int_{0}^{\infty} \sin t \sqrt{\lambda} d \sigma(\lambda) \sin \tau \sqrt{\lambda} f(\tau) d t d \tau \geq 0 .
\end{aligned}
$$

For the special choice of $k$,

$$
k(x)=\left\{\begin{aligned}
\frac{1}{2}, & x \geq 0 \\
-\frac{1}{2}, & x<0
\end{aligned}\right.
$$

the operators $K$ and $\widetilde{K}$ defined via (11.13) are identity operators on $\mathrm{L}_{2}^{n}[0 ; \ell]$.

Example 11.5 Denote by $\mathcal{F}_{\ell}$ the set of all $\mathbb{C}^{n \times n}$-valued kernels $k(x, y)$ of the form

$$
k(x, y)=s(x+y)+h(x-y) \quad(0 \leq x, y \leq \ell)
$$

where $s$ and $h$ are $\mathbb{C}^{n \times n}$-valued functions differentiable on $[0 ; 2 \ell]$ and $[-\ell ; \ell]$ respectively and such that the bounded operators $K$ and $\widetilde{K}$ acting in $\mathrm{L}_{2}^{n}[0 ; \ell]$ by the rules

$$
(K f)(x)=\int_{0}^{\ell} k(x, t) f(t) d t \quad \text { and } \quad(\widetilde{K} f)(x)=-\frac{d^{2}}{d x^{2}} \int_{0}^{\ell} k(x, t) f(t) d t
$$

are nonnegative.

The functions $s$ and $h$ in the representation (11.17) are defined by the kernel $k$ up to additive constants. Under the normalizing condition $h(0)=0$,

$$
s(x)=k\left(\frac{x}{2}, \frac{x}{2}\right) \quad \text { and } \quad h(x)=k(x, 0)-k\left(\frac{x}{2}, \frac{x}{2}\right) .
$$

Let $C$ be defined as in (11.8) and let $\Pi_{1}$ and $\Pi_{2}$ be the operators which map $\mathbb{C}^{2 n}$ into $\mathrm{L}_{2}^{n}[0 ; \ell]$ by the rules

$$
\left(\Pi_{1}\left(\begin{array}{l}
g_{1} \\
g_{2}
\end{array}\right)\right)(x)=2 k(x, 0) g_{1}+k^{\prime}(x, 0) g_{2}, \quad\left(\Pi_{2}\left(\begin{array}{c}
g_{1} \\
g_{2}
\end{array}\right)\right)(x)=g_{1}+x g_{2} \quad\left(g_{1}, g_{2} \in \mathbb{C}^{n}\right) .
$$

Using the equalities

$$
\Pi_{2} \Pi_{1}^{*} f=\Pi_{2}\left(\begin{array}{c}
\int_{0}^{\ell} k(0, t) f(t) d t \\
\int_{0}^{\ell} k^{\prime}(0, t) f(t) d t
\end{array}\right)=\int_{0}^{\ell}\left(k(0, t)+x k^{\prime}(0, t)\right) f(t) d t
$$


and

$$
\begin{aligned}
(C \widetilde{K} f)(x) & =\int_{0}^{x}(x-t) \frac{d^{2}}{d t^{2}} \int_{0}^{\ell} k(t, \xi) f(\xi) d \xi \\
& =\left.(x-t) \frac{d}{d t} \int_{0}^{\ell} k(t, \xi) f(\xi) d \xi\right|_{0} ^{x}+\left.\int_{0}^{\ell} k(t, \xi) f(\xi) d \xi\right|_{0} ^{x} \\
& =\int_{0}^{\ell} k(x, t) f(t) d t-\int_{0}^{\ell}\left(k(0, t)+x k^{\prime}(0, t)\right) f(t) d t
\end{aligned}
$$

we get identity (11.4). Applying (11.9) to the function $f(t)=t g_{2}\left(g_{2} \in \mathbb{C}^{n}\right)$ we obtain

$$
\left(C^{m}\left(t g_{2}\right)\right)(x)=\frac{(-1)^{m}}{(2 m-1) !} \int_{0}^{x}(x-t)^{2 m-1} t d t g_{2}=\frac{(-1)^{m}}{(2 m+1) !} x^{2 m+1} g_{2}
$$

which together with (11.11) and (11.19) implies

$$
\begin{aligned}
\left(\Gamma(\lambda) \Pi_{2}\left(\begin{array}{c}
g_{1} \\
g_{2}
\end{array}\right)\right)(x) & =(I-\lambda C)^{-1}\left(g_{1}+t g_{2}\right)=\sum_{j=0}^{\infty} \lambda^{j} C^{j}\left(g_{1}+t g_{2}\right) \\
& =\cos x \sqrt{\lambda} g_{1}+\frac{\sin x \sqrt{\lambda}}{\sqrt{\lambda}} g_{2},
\end{aligned}
$$

or equivalently,

$$
\left(\Gamma(\lambda) \Pi_{2} g\right)(x)=\left(\cos x \sqrt{\lambda}, \frac{\sin x \sqrt{\lambda}}{\sqrt{\lambda}}\right) g \quad\left(g \in \mathbb{C}^{2 n}\right)
$$

By Theorem 1.3 there exists a nonnegative $\mathbb{C}^{2 n \times 2 n}$-valued measure $d \sigma(\lambda)$ (despite the evidence from the previous examples, the size of a measure is more than the size of the function $k$ ) giving representations $(1.15)-(1.17)$. As before, $P_{\infty}, \widetilde{P}_{\infty}$ and $\widetilde{F}$ are zero operators. From (1.15) we get the integral representation of the kernel $k$ :

$$
k(x, y)=\int_{0}^{\infty}\left(\cos x \sqrt{\lambda}, \frac{\sin x \sqrt{\lambda}}{\sqrt{\lambda}}\right) d \sigma(\lambda)\left(\begin{array}{c}
\cos y \sqrt{\lambda} \\
\frac{\sin y \sqrt{\lambda}}{\sqrt{\lambda}}
\end{array}\right) .
$$

If the kernel $k$ of the form (11.17) is such that only the operator $K$ is nonnegative, such a representation exists with a measure supported in general, by the whole real axis ([3]).

\section{References}

[1] N. Akhieser, The Classical Moment Problem, Oliver and Boyd, London, 1965.

[2] D. Alpay, J. Ball, I. Gohberg and L. Rodman, Interpolation in the Stieltjes class, Linear Algebra and its Appl., 208-209 (1994), 485-521.

[3] V. Bolotnikov, Integral representations of the positive kernels of the form $k(t, \tau)=$ $s(t+\tau)+f(t-\tau)$, Ukr.NIINTI, preprint no. 1307 (1984). 
[4] V. Bolotnikov, Two sided Nevanlinna-Pick problem in the Stieltjes class, in: Contributions to Operator Theory and its Applications (T. Furuta, I. Gohberg and T. Nakazi, eds.), Oper. Theory: Adv. Appl., 62, Birkhäuser Verlag, Basel, 1993, pp. 15-37.

[5] V. Bolotnikov, On general moment problem on the half-axis, Linear Algebra and its Appl., 255 (1997), 57-112.

[6] M.S. Brodskii, Treugol'nye $i$ zhordanovy predstavlenija lineinykh operatorov, Nauka, Moskow, 1969. English translation: Triangular and Jordan representation of linear operators, American Math. Society, Providence, 1971.

[7] R.G. Douglas, On majorization, factorization and range inclusion of operators in Hilbert spaces, Proc. of the Amer. Math. Society, 17 (1966), 413-416.

[8] H. Dym, Book review: The commutant lifting approach to interpolation problems, by Ciprian Foias and Arthur E. Frazho, Bull. of the Amer. Math. Society, 31 (1994), 125-140.

[9] Yu. Dyukarev, Multiplicative and additive classes of Stieltjes analytic matrix-valued functions and interpolation problems associated with them, Theor. Funktsii, Func. Anal. i Prilozen., 38 (1982), 40-48.

[10] Yu. Dyukarev, Integral representations of a pair of nonnegative operators and interpolation problems in the Stieltjes class, in: Topics in Interpolation Theory (H. Dym, B. Fritzsche, V. Katsnelson and B. Kirstein, eds.), Oper. Theory Adv. Appl., OT95, Birkhäuser Verlag, Basel, 1997, pp. 165-184.

[11] Yu. Dyukarev and V. Katsnelson, Multiplicative and additive classes of Stieltjes analytic matrix-valued functions and interpolation problems associated with them, Amer. Math. Society Translations, 131 (1986), 55-70.

[12] A. V. Efimov and V. P. Potapov, J-expanding matrix functions and their role in the analytical theory of electrical circuits, Russian Math. Surveys, 28 (1973), 69-140.

[13] T. Ivanchenko and L. Sakhnovich, An operator approach to the Potapov scheme for the solution of interpolation problems, in: Matrix and Operator Valued Functions (I. Gohberg and L.A. Sakhnovich, eds.), Oper. Theory Adv. Appl., OT72, Birkhäuser Verlag, Basel, 1994, pp. 48-86.

[14] I. S. Kats and M. G. Krein, R-functions - analytic functions mapping the upper halfplane into itself, Amer. Math. Society Transl., 103 (1973), 1-18.

[15] V. Katsnelson. Methods of J-theory in continuous interpolation problems of analysis. Private translation of T. Ando, Sapporo, 1982.

[16] V. Katsnelson. Regularization of the fundamental matrix inequality in the problem of the decomposition of a positive kernel into elementary kernels, Dokl. Ukr. SSR, 3 (1984), $6-8$. 
[17] V. Katsnelson. Continuous analogues of the Hamburger-Nevanlinna theorem and fundamental matrix inequalities, Amer. Math. Soc. Transl., 136 (1987), 49-96.

[18] V. Katsnelson, A transformation of Potapov's fundamental matrix inequality, in: Topics in Interpolation Theory (H. Dym, B. Fritzsche, V. Katsnelson and B. Kirstein, eds.), Oper. Theory Adv. Appl., OT95, Birkhäuser Verlag, Basel, 1997, pp. 253-281.

[19] V. Katsnelson, A. Kheifets and P. Yuditskii, An abstract interpolation problem and extension theory of isometric operators, in: Operators in spaces of functions and problems in the function theory (V.A. Marchenko, ed.), 146, Naukova dumka, Kiev, 1987, pp. 83-96. English transl. in: Topics in Interpolation Theory (H. Dym, B. Fritzsche, V. Katsnelson and B. Kirstein, eds.), Oper. Theory Adv. Appl., OT95, Birkhäuser Verlag, Basel, 1997, pp. 283-298.

[20] M.G. Kreĭn, On a general method of decomposing hermite-positive nuclei into elementary products, Dokl. Akad. Nauk. SSSR, 53 (1946), 3-6.

[21] M.G. Kreı̆n and A.A. Nudelman, The Markov moment problem and extremal problems, Translations of Mathematical Monographs 50, Amer. Math. Soc., Providence, Rhode Island , 1977.

[22] A.A. Nudelman, On a new problem of moment problem type, Sov. Math. Dokl., 18 (1977), 507-510.

[23] L.A. Sahknovich, Method of operator identities and problems of analysis, St. Petersburg Math. Journal, 5 (1994), 1-69.

[24] L.A. Sakhnovich, Factorization problems and operator identities, Russian Math. Surveys, 41 (1986), 1-64.

Vladimir Bolotnikov

Department of Theoretical Mathematics

The Weizmann Institute of Science

Rehovot 76100, Israel
Lev A. Sakhnovich

Department of Mathematics

Ukranian Academy of Communications

Odessa 270111, Ukraine

AMS classification 47A57, 30E05 